Supporting Information

For

\title{
Synthesis of Imides, Imidates, Amidines, and Amides by Intercepting Aryne-Isocyanide Adduct with Weak Nucleophiles
}

\author{
Sourav Ghorai and Daesung Lee* \\ Department of Chemistry, University of Illinois at Chicago \\ E-mail: dsunglee@uic.edu
}

\section{Contents}

General Information $\quad$ S1

$\begin{array}{ll}\text { Experimental Details and General Procedures } & \text { S2-S7 }\end{array}$

Characterization Data of Substrates $\quad$ S8-S9

$\begin{array}{ll}\text { Characterization Data of Products } & \text { S9-S18 }\end{array}$

${ }^{1} \mathrm{H}$ and ${ }^{13} \mathrm{C}$ NMR Spectra of Substrates S19-S26

${ }^{1} \mathrm{H}$ and ${ }^{13} \mathrm{C}$ NMR Spectra of Products $\quad$ S27-S69 
Experimental section: All reactions were carried out in oven or flame-dried glassware unless otherwise noted. Reagents which were commercially available, were purchased from Sigma Aldrich, Alfa Aesar, Acros, and Oakwood Products unless otherwise noted. Known compounds were prepared according to literature procedure. Anhydrous acetonitrile from Sigma-Aldrich was distilled over calcium hydride $\left(\mathrm{CaH}_{2}\right)$ under nitrogen atmosphere. Acetic acid was purchased from Fischer Scientific. Column chromatography was performed using silica gel $60 \AA$ (32-63 mesh) purchased from Silicycle Inc. Analytical thin layer chromatography (TLC) was performed on $0.25 \mathrm{~mm}$ E. Merck precoated silica gel 60 (particle size $0.040-0.063 \mathrm{~mm}$ ). Yield was calculated on basis of chromatographically and spectroscopically pure isolated compound. ${ }^{1} \mathrm{H} \mathrm{NMR}$ and ${ }^{13} \mathrm{C}$ NMR spectra were recorded on a Bruker AV-500 spectrometer at $298 \mathrm{~K}$, unless otherwise stated. ${ }^{1} \mathrm{H}$ NMR chemical shifts $(\delta)$ were reported in parts per million $(\mathrm{ppm})$ downfield of TMS and were referenced relative to the residual proteated solvent peak $\left(\mathrm{CDCl}_{3}(7.26 \mathrm{ppm})\right) \cdot{ }^{13} \mathrm{C}$ chemical shifts $(\delta)$ were reported in parts per million downfield of TMS and are referenced to the carbon resonance of the solvent $\left(\mathrm{CDCl}_{3}, \delta 77.2 \mathrm{ppm}\right)$. Multiplicities in ${ }^{1} \mathrm{H}$ NMR were abbreviated by $\mathrm{s}$ (singlet), d (doublet), t (triplet), q (quartet), quin (quintet), sext (sextet), sept (septet) or $\mathrm{m}$ (multiplet). ${ }^{1} \mathrm{H}$ NMR signals that fall within a ca. $0.3 \mathrm{ppm}$ range are generally reported as a multiplet, with a range of chemical shift values corresponding to the peak or center of the peak. Coupling constants, $J$, are reported in $\mathrm{Hz}$ (Hertz). Electrospray ionization (ESI) mass spectra were recorded on a Waters Micromass Q-Tof Ultima (Waters Corporation, Milford, MA, USA) at the University of Illinois at Urbana-Champaign. Electron impact (EI) mass spectra and Chemical Ionization $(\mathrm{Cl})$ mass spectra were obtained using a Micromass 70-VSE (Waters Corporation, Milford, MA, USA) at the University of Illinois at Urbana-Champaign. 


\section{General Procedure for Synthesis of symmetrical bis-1,3-diyne}

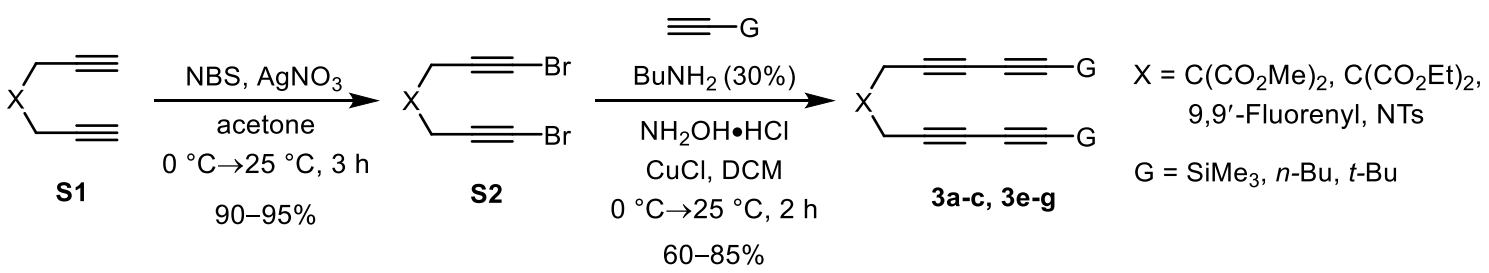

To a stirred solution of diyne $(10.0 \mathrm{mmol})$ in acetone at $0{ }^{\circ} \mathrm{C}, \mathrm{N}$-bromosuccinimide $(25.0 \mathrm{mmol})$ and $\mathrm{AgNO}_{3}(1.0 \mathrm{mmol})$ were added sequentially under $\mathrm{N}_{2}$ atmosphere in the dark. ${ }^{1}$ After addition, the ice bath was removed and stirring was continued for additional $3 \mathrm{~h}$. Upon complete consumption of alkyne, the reaction mixture was concentrated under reduced pressure and filtered through silica gel. Purification by flash column chromatography (SiO, EtOAc-hexanes, 1:5 to $1: 3)$ provided dibromide (S2) in excellent yield.

To a mixture of $30 \%$ aq. $n-\mathrm{BuNH}_{2}(10 \mathrm{~mL}, 2 \mathrm{~mL}$ per $1.0 \mathrm{mmol}$ of terminal alkyne) and $\mathrm{CuCl}(1.5$ $\mathrm{mmol}$ ) in a two-necked round-bottom flask, a solution of terminal alkyne $(5.0 \mathrm{mmol})$ in $\mathrm{CH}_{2} \mathrm{Cl}_{2}$ was added slowly at $0{ }^{\circ} \mathrm{C}$ under $\mathrm{N}_{2}$ atmosphere. ${ }^{2}$ Then $\mathbf{S 2}(2.0 \mathrm{mmol})$ diluted in $\mathrm{CH}_{2} \mathrm{Cl}_{2}$ was added dropwise into the reaction mixture over 30-45 $\mathrm{min}$. After $5 \mathrm{~min}$, the ice bath was removed and stirring was continued for additional $1-2 \mathrm{~h}$ at room temperature (TLC monitoring). A pinch of $\mathrm{NH}_{2} \mathrm{OH} \cdot \mathrm{HCl}$ was added several times into the reaction mixture when the solution becomes blue. The reaction was quenched by a sat. solution of $\mathrm{NH}_{4} \mathrm{Cl}$ and extracted with $\mathrm{CH}_{2} \mathrm{Cl}_{2}$. The combined organic extracts were washed with water and brine sequentially and dried over anhydrous $\mathrm{Na}_{2} \mathrm{SO}_{4}$ and concentrated under reduced pressure. The crude material was purified by flash column chromatography (SiO, EtOAc-hexanes, 1:20 to 1:10) to isolate pure symmetrical bis-1,3diyne in moderate to good yield.

\section{Procedure for Synthesis of a Triynes with a Phenylamide Linker (3d)}

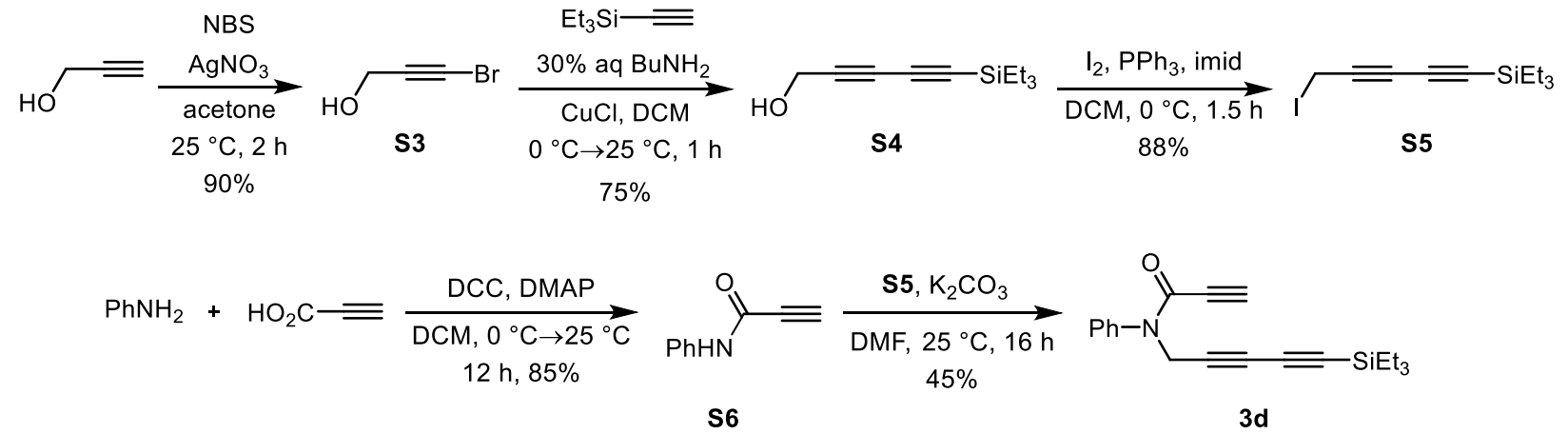

To a stirred solution of 2-propyn-1-ol $(5.0 \mathrm{~g}, 89.1 \mathrm{mmol})$ in acetone at $0{ }^{\circ} \mathrm{C}, \mathrm{N}$-bromosuccinimide $(19.8 \mathrm{~g}, 111.5 \mathrm{mmol})$ and $\mathrm{AgNO}_{3}(1.5 \mathrm{~g}, 8.9 \mathrm{mmol})$ were added sequentially under $\mathrm{N}_{2}$ atmosphere in the dark. After addition, the ice bath was removed and stirring was continued for additional 2 $\mathrm{h}$ at room temperature. Upon complete consumption of alkyne, the reaction mixture was concentrated under reduced pressure and filtered through silica gel. Purification by flash column 
chromatography (SiO, EtOAc-hexanes, 1:5) provided pure bromide $\mathbf{S 3}$ in excellent yield $(7.9 \mathrm{~g}$, $90 \%)$.

To a mixture of $30 \%$ aq. $n-\mathrm{BuNH}_{2}(22.2 \mathrm{~mL})$ and $\mathrm{CuCl}(331 \mathrm{mg}, 3.4 \mathrm{mmol})$ in a two-necked roundbottom flask, a solution of triethyl(ethynyl)silane $(1.6 \mathrm{~g}, 11.2 \mathrm{mmol})$ in $\mathrm{CH}_{2} \mathrm{Cl}_{2}$ was added slowly at $0{ }^{\circ} \mathrm{C}$ under $\mathrm{N}_{2}$ atmosphere. Then $\mathbf{S 3}(1.0 \mathrm{~g}, 7.4 \mathrm{mmol})$ diluted in $\mathrm{CH}_{2} \mathrm{Cl}_{2}$ was added dropwise into the reaction mixture over $30 \mathrm{~min}$. After $5 \mathrm{~min}$, the ice bath was removed and stirring was continued for additional $1 \mathrm{~h}$. A pinch of $\mathrm{NH}_{2} \mathrm{OH} \cdot \mathrm{HCl}$ was added several times into the reaction mixture when the solution becomes blue. The reaction was quenched by a sat. solution $\mathrm{NH}_{4} \mathrm{Cl}$ and extracted with $\mathrm{CH}_{2} \mathrm{Cl}_{2}$. The combined organic extracts were washed with water and brine sequentially and dried over anhydrous $\mathrm{Na}_{2} \mathrm{SO}_{4}$ and concentrated under reduced pressure. The crude material was purified by flash column chromatography (SiO, EtOAc-hexanes, 1:10) to isolate pure 2,4-diyne-1ol S4 (1.1 g, 75\%).

To a stirred solution of 2,4-diyne-1ol $\mathbf{S 4}(0.8 \mathrm{~g}, 4.1 \mathrm{mmol})$ in $\mathrm{CH}_{2} \mathrm{Cl}_{2}$ at $0{ }^{\circ} \mathrm{C}, \mathrm{PPh}_{3}(1.2 \mathrm{~g}, 4.5 \mathrm{mmol})$, $\mathrm{I}_{2}(1.2 \mathrm{~g}, 4.5 \mathrm{mmol})$, and imidazole $(308 \mathrm{mg}, 4.5 \mathrm{mmol})$ were added sequentially under $\mathrm{N}_{2}$ atmosphere and continued stirring at same temperature. ${ }^{3}$ After $1.5 \mathrm{~h}$, the reaction mixture was quenched by a sat. solution of $\mathrm{Na}_{2} \mathrm{~S}_{2} \mathrm{O}_{3}$ and extracted with $\mathrm{CH}_{2} \mathrm{Cl}_{2}$. The combined organic extracts were washed with water and brine sequentially and dried over anhydrous $\mathrm{Na}_{2} \mathrm{SO}_{4}$ and concentrated under reduced pressure. The crude material was purified by flash column chromatography (SiO, EtOAc-hexanes, 1:20) to isolate pure propagyl iodide S5 (1.1 g, 88\%).

To a stirred solution of aniline $(1.0 \mathrm{~g}, 10.7 \mathrm{mmol})$ and propiolic acid $(828 \mathrm{mg}, 11.8 \mathrm{mmol})$ in $\mathrm{Et}_{2} \mathrm{O}$ at $0{ }^{\circ} \mathrm{C}, \mathrm{DCC}(2.7 \mathrm{~g}, 12.9 \mathrm{mmol})$ and DMAP $(131 \mathrm{mg}, 1.1 \mathrm{mmol})$ were added sequentially under $\mathrm{N}_{2}$ atmosphere. ${ }^{4}$ After addition, the ice bath was removed and stirring was continued for $12 \mathrm{~h}$ at 25 ${ }^{\circ} \mathrm{C}$. Upon complete consumption of aniline, the reaction mixture was concentrated under reduced pressure and purified by flash column chromatography (SiO, EtOAc-hexanes, 1:3) to isolate pure phenylamide $\mathbf{S 6}$ (1.3 g, 85\%).

To a stirred solution of phenylamide $\mathbf{S 6}(286 \mathrm{mg}, 2.0 \mathrm{mmol})$, and propagyl iodide $\mathbf{S 5}$ (500 mg, 1.6 $\mathrm{mmol}$ ) in DMF at $25^{\circ} \mathrm{C}, \mathrm{K}_{2} \mathrm{CO}_{3}\left(452 \mathrm{mg}, 3.3 \mathrm{mmol}\right.$ ) was added under $\mathrm{N}_{2}$ atmosphere. ${ }^{5}$ After $16 \mathrm{~h}$, the reaction mixture was diluted by water and extracted with EtOAc (X3). The combined organic extracts were washed with water and brine sequentially and dried over anhydrous $\mathrm{Na}_{2} \mathrm{SO}_{4}$ and concentrated under reduced pressure. The crude material was purified by flash column chromatography (SiO, EtOAc-hexanes, 1:3) to isolate pure triyne 3d in moderate yield (236 mg, $45 \%)$. 


\section{Procedure for Synthesis of a Triyne with a Benzoyl Linker (3h)}

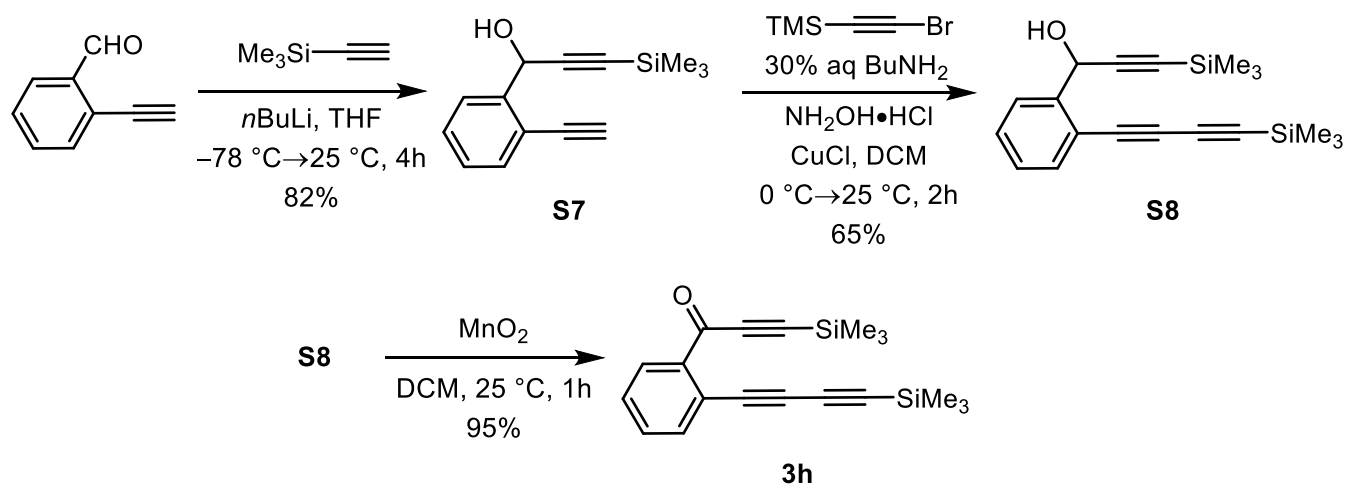

To a stirred solution of ethynyltrimethylsilane $(588 \mathrm{mg}, 6.0 \mathrm{mmol})$ in dry THF was added BuLi ( 2.2 $\mathrm{mL}, 2.5 \mathrm{M}$ in hexane, $5.5 \mathrm{mmol}$ ) slowly at $-78{ }^{\circ} \mathrm{C}$ under $\mathrm{N}_{2}$ atmosphere. After $1 \mathrm{~h}, 2-$ ethynylbenzaldehyde $(650 \mathrm{mg}, 5.0 \mathrm{mmol}$ ) diluted in THF was added dropwise at same temperature. After $30 \mathrm{~min}$, the reaction mixture was gradually warmed up to room temperature and the reaction mixture was further stirred for 30 min before it was quenched with sat. $\mathrm{NH}_{4} \mathrm{Cl}$ and extracted with EtOAc (X3). The combined organic extracts were washed with water and brine sequentially and dried over anhydrous $\mathrm{Na}_{2} \mathrm{SO}_{4}$ and concentrated under reduced pressure. The crude material was purified by flash column chromatography (SiO, EtOAc-hexanes, 1:10) to isolate pure diyne $\mathbf{S 7}$ (935 $\mathrm{mg}, 82 \%$ ).

To a mixture of $30 \%$ aq. $n$-BuNH $2(4.6 \mathrm{~mL})$ and $\mathrm{CuCl}(67 \mathrm{mg}, 0.7 \mathrm{mmol})$ in a two-necked roundbottom flask, a solution of $\mathbf{S 7}(520 \mathrm{mg}, 2.3 \mathrm{mmol})$ in $\mathrm{CH}_{2} \mathrm{Cl}_{2}$ was added slowly at $0{ }^{\circ} \mathrm{C}$ under $\mathrm{N}_{2}$ atmosphere. Then (bromoethynyl)trimethylsilane $\left(605 \mathrm{mg}, 3.4 \mathrm{mmol}\right.$ ) diluted in $\mathrm{CH}_{2} \mathrm{Cl}_{2}$ was added dropwise into the reaction mixture over $30 \mathrm{~min}$. After $5 \mathrm{~min}$, the ice bath was removed and stirring was continued for additional $1 \mathrm{~h}$ at room temperature. A pinch of $\mathrm{NH}_{2} \mathrm{OH} \cdot \mathrm{HCl}$ was added several times into the reaction mixture when the solution becomes blue. The reaction was quenched by a sat. solution of $\mathrm{NH}_{4} \mathrm{Cl}$ and extracted with $\mathrm{CH}_{2} \mathrm{Cl}_{2}$. The combined organic extracts were washed with water and brine sequentially and dried over anhydrous $\mathrm{Na}_{2} \mathrm{SO}_{4}$ and concentrated under reduced pressure. The crude material was purified by flash column chromatography (SiO, EtOAc-hexanes, 1:15) to isolate pure triyne $\mathbf{S 8 ~ ( 4 8 0 ~} \mathbf{m g}, 65 \%$ ).

To a stirred solution of $\mathbf{S 8}(480 \mathrm{mg}, 1.5 \mathrm{mmol})$ in $\mathrm{CH}_{2} \mathrm{Cl}_{2}, \mathrm{MnO}_{2}(645 \mathrm{mg}, 7.4 \mathrm{mmol})$ was added in one portion under $\mathrm{N}_{2}$ atmosphere at room temperature. After $2 \mathrm{~h}$, the reaction mixture was filtered through celite and concentrated under reduced pressure. The crude $3 \mathrm{~h}$ ( $453 \mathrm{mg}, 95 \%)$ was subjected to the next reaction without further purification. 


\section{General Procedure for the Synthesis of Aromatic Isocyanide ${ }^{6}$}

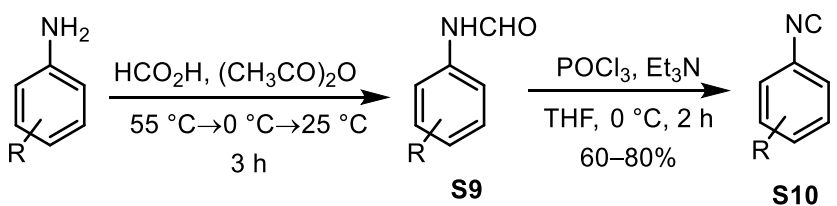

A mixture of $\mathrm{HCO}_{2} \mathrm{H}(4.0 \mathrm{mmol})$ and $\left(\mathrm{CH}_{3} \mathrm{CO}\right)_{2} \mathrm{O}(4.0 \mathrm{mmol})$ were stirred for $2 \mathrm{~h}$ at $55^{\circ} \mathrm{C}$. After cooling down the reaction mixture at $0{ }^{\circ} \mathrm{C}$, aniline $(2.0 \mathrm{mmol})$ was added slowly and continued stirring for additional $2 \mathrm{~h}$ at room temperature. After complete consumption of aniline, all the volatiles were removed from the reaction mixture under reduced pressure and the crude formamide $\mathbf{S 9}$ was subjected to the next reaction without further purification.

To a stirred solution of formamide in dry $\mathrm{THF}$ at $0{ }^{\circ} \mathrm{C}$ were added $\mathrm{Et}_{3} \mathrm{~N}(10.0 \mathrm{mmol})$ and $\mathrm{POCl}_{3}(2.4$ $\mathrm{mmol}$ ) dropwise sequentially. After additional stirring for $2 \mathrm{~h}$ at $0{ }^{\circ} \mathrm{C}$, the reaction was quenched with water and extracted with EtOAc (X2). The combined organic layers were washed with water and brine sequentially and dried over anhydrous $\mathrm{Na}_{2} \mathrm{SO}_{4}$ and concentrated under reduced pressure. The crude material was purified by flash column chromatography (SiO, EtOAc-hexanes, 1:20 to $1: 10$ ) to isolate pure isocyanide $\mathbf{S 1 0 .}$

\section{Stoichiometry Screening for Three-component Coupling between Aryne, Isocyanide, and Carboxylic Acid}

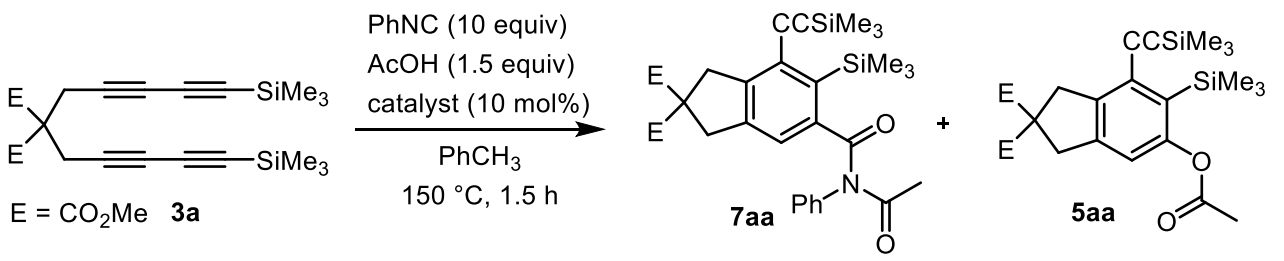

\begin{tabular}{cccccc}
\hline entry & PhNC & $\mathrm{AcOH}$ & catalyst & 7aa:5aa & 7aa $(\%)^{a}$ \\
\hline 1 & 1.5 equiv & 1.5 equiv & $\mathrm{AgSbF}_{6}$ & $0.3: 1.0$ & $10 \%$ \\
2 & 5 equiv & 1.5 equiv & $\mathrm{AgSbF}_{6}$ & $1.5: 1.0$ & $38 \%$ \\
3 & 10 equiv & 1.5 equiv & $\mathrm{AgSbF}_{6}$ & $5.9: 1.0$ & $68 \%$ \\
4 & 10 equiv & 1.1 equiv & $\mathrm{AgSbF}_{6}$ & $6.7: 1.0$ & $49 \%$ \\
5 & 10 equiv & 1.5 equiv & - & $26.2: 1.0$ & $84 \%$ \\
6 & 5 equiv & 1.5 equiv & - & $10.4: 1.0$ & $72 \%$ \\
7 & 1.5 equiv & 1.5 equiv & - & $3.0: 1.0$ & $57 \%$ \\
\hline
\end{tabular}

${ }^{a}$ NMR yield using dibromomethane as internal standard.

To maximize arylimide product from three-component coupling between aryne, isocyanide, and carboxylic acid, reactions with various stoichiometry of isocyanide and carboxylic acid were 
screened. In a typical procedure, malonate ester-tethered tetrayne $3 a(0.1 \mathrm{mmol})$, phenyl isocyanide, and acetic acid were taken in a thick-walled $25 \mathrm{~mL}$ Schlenk tube in $3 \mathrm{~mL}$ dry toluene. After flushing with nitrogen several times, the Schlenk tube was placed in a heated oil bath (150 ${ }^{\circ} \mathrm{C}$ ) for $1.5 \mathrm{~h}$. Upon completion, the reaction mixture was concentrated under reduced pressure, and NMR yield was calculated using dibromomethane as an internal standard. These results indicate that an excess amount of PhNC (10 equiv) is necessary for improving the selectivity between imide 7aa (three-component coupling product) and ester (5aa) (aryne-carboxylic acid adduct).

\section{Procedure A: Preparation of N-Aryl Imides}

Multiyne substrate $3(0.1 \mathrm{mmol})$, isocyanide $(1.0 \mathrm{mmol})$, carboxylic acid $(0.15 \mathrm{mmol})$, and $\mathrm{AgSbF} 6$ ( $0.01 \mathrm{mmol})$ were taken in a thick-walled $25 \mathrm{~mL}$ Schlenk tube in $3 \mathrm{~mL}$ dry toluene. After flushing several times with nitrogen, the Schlenk tube was placed in a heated oil bath $\left(150{ }^{\circ} \mathrm{C}\right)$ for $1.5 \mathrm{~h}$. Upon completion, the reaction mixture was concentrated under reduced pressure, and the crude material was purified by flash column chromatography (SiO, EtOAc-hexanes, 1:10 to 1:3) to isolate pure $\mathrm{N}$-aryl imide product.

\section{Procedure B: Preparation of N-Aryl Imidates}

Multiyne substrate $3(0.1 \mathrm{mmol})$, isocyanide $(0.2 \mathrm{mmol})$, and alcohol $(0.25 \mathrm{mmol})$ were taken in a thick-walled $25 \mathrm{~mL}$ Schlenk tube in $3 \mathrm{~mL}$ dry toluene. After flushing several times with nitrogen, the Schlenk tube was placed in a heated oil bath $\left(150^{\circ} \mathrm{C}\right)$ for $1.5 \mathrm{~h}$. Upon completion, the reaction mixture was concentrated under reduced pressure, and the crude material was purified by flash column chromatography (SiO, EtOAc-hexanes, 1:10 to 1:3) to isolate pure $\mathrm{N}$-aryl imidate product.

\section{Procedure C: Preparation of N-Aryl Amidines}

Multiyne substrate $3(0.1 \mathrm{mmol})$, isocyanide $(0.2 \mathrm{mmol})$, and sulfonamide $(0.15 \mathrm{mmol})$ were taken in a thick-walled $25 \mathrm{~mL}$ Schlenk tube in $3 \mathrm{~mL}$ dry toluene. After flushing several times with nitrogen, the Schlenk tube was placed in a heated oil bath $\left(150^{\circ} \mathrm{C}\right)$ for $1.5 \mathrm{~h}$. Upon completion, the reaction mixture was concentrated under reduced pressure, and the crude material was purified by flash column chromatography (SiO, EtOAc-hexanes, 1:10 to 1:3) to isolate pure N-aryl amidine product.

\section{Procedure D: Preparation of N-Aryl Amides}

Multiyne substrate $3(0.1 \mathrm{mmol})$, isocyanide $(0.2 \mathrm{mmol})$, and $\mathrm{H}_{2} \mathrm{O}(1.0 \mathrm{mmol})$ were taken in a thick-walled $25 \mathrm{~mL}$ Schlenk tube in $3 \mathrm{~mL}$ dry toluene. After flushing several times with nitrogen, the Schlenk tube was placed in a heated oil bath $\left(150^{\circ} \mathrm{C}\right)$ for $1.5 \mathrm{~h}$. Upon completion, the reaction mixture was concentrated under reduced pressure, and the crude material was purified by flash column chromatography (SiO, EtOAc-hexanes, $1: 10$ to 1:3) to isolate pure $\mathrm{N}$-aryl amide product.

\section{Procedure E: Preparation of $\boldsymbol{N}$-aryl imidate (8ac) at $1.0 \mathrm{mmol}$ Scale}

Multiyne 3a (400 mg, $1.0 \mathrm{mmol})$, phenyl isocyanide $(206 \mathrm{mg}, 2.0 \mathrm{mmol}$ ), and phenol (235 mg, 2.5 $\mathrm{mmol}$ ) were taken in a thick-walled $25 \mathrm{~mL}$ Schlenk tube in $12 \mathrm{~mL}$ dry toluene. After flushing with 
nitrogen several times, the Schlenk tube was placed in a heated oil bath $\left(150^{\circ} \mathrm{C}\right)$ for $1.5 \mathrm{~h}$. Upon completion, the reaction mixture was concentrated under reduced pressure, and the crude material was purified by flash column chromatography (SiO, EtOAc-hexanes, 1:10 to 1:3) to isolate pure $N$-aryl imidate 8 ac ( $519 \mathrm{mg}, 87 \%$ yield).

\section{Procedure F: Reaction with Deuterated Pro-nucleophile (AcOH- $\left.d_{4}\right)$}
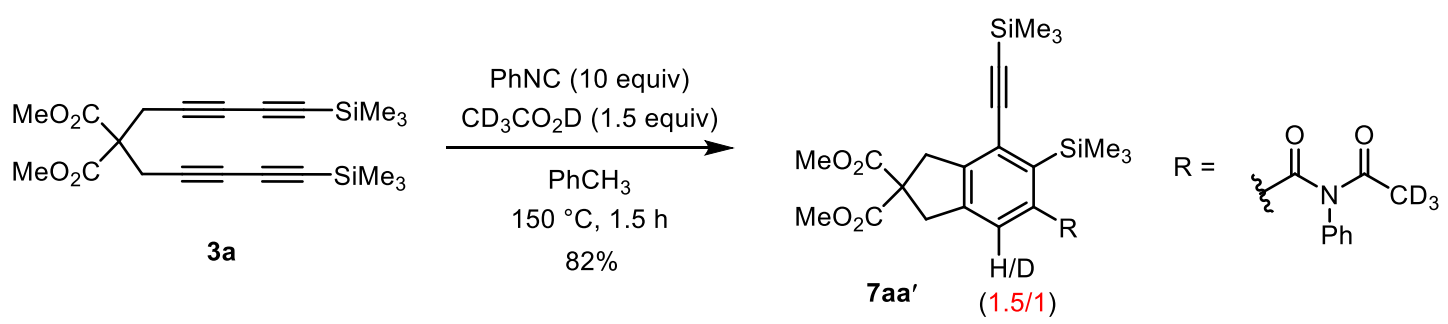

Multiyne $3 a(0.1 \mathrm{mmol})$, phenyl isocyanide $(1.0 \mathrm{mmol})$, and acetic acid- $d_{4}(0.15 \mathrm{mmol})$ were taken in a thick-walled $25 \mathrm{~mL}$ Schlenk tube in $3 \mathrm{~mL}$ dry toluene. After flushing with nitrogen several times, the Schlenk tube was placed in a heated oil bath $\left(150^{\circ} \mathrm{C}\right)$ for $1.5 \mathrm{~h}$. Upon completion, the reaction mixture was concentrated under reduced pressure, and the crude material was purified by flash column chromatography (SiO, EtOAc-hexanes, $1: 10$ to $1: 3$ ) to isolate pure deuterated $N$ aryl imide 7aa' (23.6 mg, 82\% yield). This unexpectedly low deuterium incorporation (40\%) might be the consequence of $\mathrm{H}-\mathrm{D}$ exchange with adventitious $\mathrm{H}_{2} \mathrm{O}$ in the reaction medium. Using freshly distilled solvent did not change the ratio significantly. Adding $4 \AA$ molecular sieve (powder) to the reaction mixture further increased proton incorporation over deuterium, providing only $20 \%$ deuterium-incorporated product.

\section{References:}

[1] Hofmeister, H.; Annen, K.; Laurent, H.; Wiechert, H. Angew. Chem., Int. Ed. 1984, 23, 727.

[2] a) Chodkiewicz, W. Ann. Chim. 1957, 2, 819; b) Cadiot, P.; Chodkiewicz, W. in Chemistry of

Acetylenes (Ed.: H. G. Viehe), Marcel Dekker, New York, 1969, pp. 597-647.

[3] Garegg, P. J.; Samuelsson, B. J. Chem. Soc., Chem. Commun. 1979, 978.

[4] Neises, B.; Steglich, W. Org. Synth. 1985, 63, 183.

[5] Karmakar, R.; Yun, S. Y.; Chen, J.; Xia, Y.; Lee, D. Angew. Chem., Int. Ed. 2015, 54, 6582.

[6] a) Okandeji, B. O.; Greenwald, D. M.; Wroten, J.; Sello, J. K. Bioorg. Med. Chem. 2011, 19, 7679; b) Yan, X.; Zhang, S.; Zhang, P.; Wu, X.; Liu, A.; Guo, G.; Dong, Y.; Li, X. Angew. Chem., Int. Ed. 2018, 57, 8947; c) Škoch, K.; Císařová, I.; Štěpnička, P. Chem. - Eur. J. 2018, 24, 13788. 


\section{Characterization Data for Substrates}

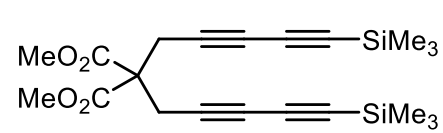

$3 \mathbf{a}$

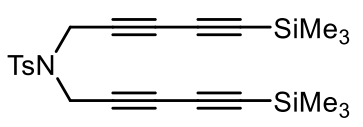

$3 \mathbf{b}$
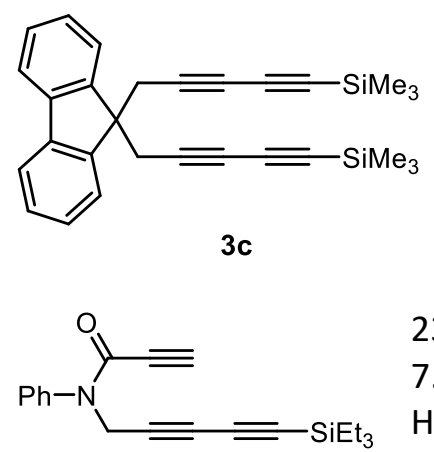

$3 d$
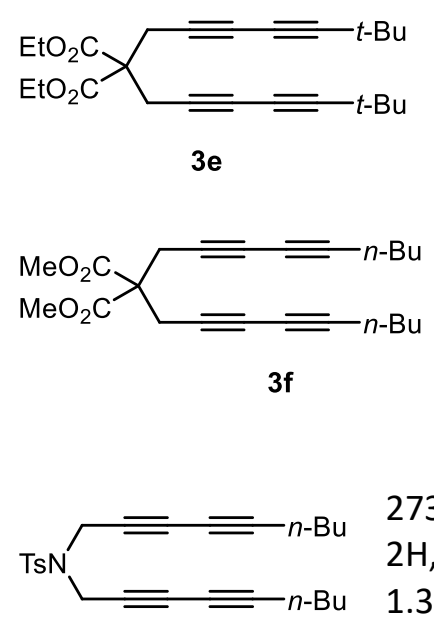

$3 g$

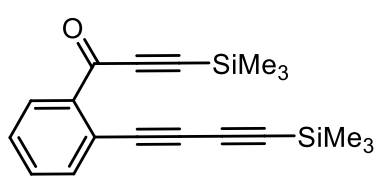

3h
$746 \mathrm{mg}$, white solid. ${ }^{1} \mathrm{H}$ NMR $\left(\mathrm{CDCl}_{3}, 500 \mathrm{MHz}\right): \delta 3.77(\mathrm{~s}, 6 \mathrm{H}), 3.04(\mathrm{~s}, 4 \mathrm{H})$, $0.18(\mathrm{~s}, 18 \mathrm{H}) ;{ }^{13} \mathrm{C} \mathrm{NMR}\left(\mathrm{CDCl}_{3}, 125 \mathrm{MHz}\right): \delta 168.4,87.7,84.8,72.8,68.8,56.4$, 53.3, 23.8, -0.5; HRMS (ESI) calcd for $\mathrm{C}_{21} \mathrm{H}_{29} \mathrm{O}_{4} \mathrm{Si}_{2}[\mathrm{M}+\mathrm{H}]^{+}$401.1604, found 401.1612 .

$460 \mathrm{mg}$, white solid. ${ }^{1} \mathrm{H}$ NMR $\left(\mathrm{CDCl}_{3}, 500 \mathrm{MHz}\right): \delta 7.68(\mathrm{~d}, 2 \mathrm{H}, J=8.2 \mathrm{~Hz}), 7.32$ (d, $2 \mathrm{H}, J=8.2 \mathrm{~Hz}), 4.18(\mathrm{~s}, 4 \mathrm{H}), 2.42(\mathrm{~s}, 3 \mathrm{H}), 0.18(\mathrm{~s}, 18 \mathrm{H}) ;{ }^{13} \mathrm{C} \mathrm{NMR}\left(\mathrm{CDCl}_{3}, 125 \mathrm{MHz}\right)$ : $\delta$ 144.3, 134.5, 129.8, 127.9, 87.0, 86.9, 71.0, 70.1, 37.3, 21.6, -0.5; HRMS (ESI) calcd for $\mathrm{C}_{23} \mathrm{H}_{30} \mathrm{NO}_{2} \mathrm{SSi}_{2}[\mathrm{M}+\mathrm{H}]^{+} 440.1536$, found 440.1531 .

$378 \mathrm{mg}$, white solid. ${ }^{1} \mathrm{H}$ NMR $\left(\mathrm{CDCl}_{3}, 500 \mathrm{MHz}\right): \delta 7.77-7.67(\mathrm{~m}, 4 \mathrm{H}), 7.40(\mathrm{t}$, $2 \mathrm{H}, J=7.2 \mathrm{~Hz}), 7.35(\mathrm{t}, 2 \mathrm{H}, J=7.2 \mathrm{~Hz}), 2.89(\mathrm{~s}, 4 \mathrm{H}), 0.21(\mathrm{~s}, 18 \mathrm{H}) ;{ }^{13} \mathrm{C}$ NMR $\left(\mathrm{CDCl}_{3}, 125 \mathrm{MHz}\right): \delta 147.8,139.7,128.3,127.6,123.9,120.1,88.2,84.2,76.1$, 68.0, 50.4, 28.4, -0.3; HRMS (EI) calcd for $\mathrm{C}_{29} \mathrm{H}_{30} \mathrm{Si}_{2}$ [M] 434.18861, found 434.18865.

$235 \mathrm{mg}$, light yellow oil. ${ }^{1} \mathbf{H}$ NMR $\left(\mathrm{CDCl}_{3}, 500 \mathrm{MHz}\right): \delta 7.46-7.37(\mathrm{~m}, 3 \mathrm{H}), 7.36-$ $7.31(\mathrm{~m}, 2 \mathrm{H}), 4.56(\mathrm{~s}, 2 \mathrm{H}), 2.83(\mathrm{~s}, 1 \mathrm{H}), 0.97(\mathrm{t}, 9 \mathrm{H}, J=7.9 \mathrm{~Hz}), 0.60(\mathrm{q}, 6 \mathrm{H}, J=7.9$ $\mathrm{Hz}) ;{ }^{13} \mathrm{C} \mathrm{NMR}\left(\mathrm{CDCl}_{3}, 125 \mathrm{MHz}\right): \delta$ 152.5, 140.3, 129.5, 129.0, 128.2, 88.3, 84.7, 80.6, 75.6, 71.4, 69.5, 38.7, 7.3, 4.1; HRMS (ESI) calcd for $\mathrm{C}_{20} \mathrm{H}_{24} \mathrm{NOSi}[\mathrm{M}+\mathrm{H}]^{+}$ 322.1627, found 322.1637.

$281 \mathrm{mg}$, white solid. ${ }^{1} \mathrm{H}$ NMR $\left(\mathrm{CDCl}_{3}, 500 \mathrm{MHz}\right): \delta 4.22(\mathrm{q}, 4 \mathrm{H}, J=7.1 \mathrm{~Hz}), 3.04$ $(\mathrm{s}, 4 \mathrm{H}), 1.25(\mathrm{t}, 6 \mathrm{H}, J=7.1 \mathrm{~Hz}), 1.20(\mathrm{~s}, 18 \mathrm{H}) ;{ }^{13} \mathrm{C} \mathrm{NMR}\left(\mathrm{CDCl}_{3}, 125 \mathrm{MHz}\right): \delta$ $168.4,86.2,71.8,68.5,63.6,62.2,56.6,30.5,27.9,23.7,14.0$; HRMS (ESI) calcd for $\mathrm{C}_{25} \mathrm{H}_{33} \mathrm{O}_{4}[\mathrm{M}+\mathrm{H}]^{+} 397.2379$, found 397.2377 .

$246 \mathrm{mg}$, white solid. ${ }^{1} \mathbf{H}$ NMR $\left(\mathrm{CDCl}_{3}, 500 \mathrm{MHz}\right): \delta 3.73(\mathrm{~s}, 6 \mathrm{H}), 3.02(\mathrm{~s}, 4 \mathrm{H})$, $2.21(\mathrm{t}, 4 \mathrm{H}, J=6.6 \mathrm{~Hz}), 1.52-1.30(\mathrm{~m}, 8 \mathrm{H}), 0.88(\mathrm{t}, 6 \mathrm{H}, J=7.2 \mathrm{~Hz}) ;{ }^{13} \mathrm{C} \mathbf{N M R}$ ( $\left.\mathrm{CDCl}_{3}, 125 \mathrm{MHz}\right): \delta 168.7,78.9,70.4,68.7,64.8,56.7,53.2,30.2,23.7,21.9$, 18.8, 13.5; HRMS (ESI) calcd for $\mathrm{C}_{23} \mathrm{H}_{29} \mathrm{O}_{4}[\mathrm{M}+\mathrm{H}]^{+} 369.2066$, found 369.2076.

$273 \mathrm{mg}$, colorless oil. ${ }^{1} \mathrm{H}$ NMR $\left(\mathrm{CDCl}_{3}, 500 \mathrm{MHz}\right): \delta 7.64(\mathrm{~d}, 2 \mathrm{H}, J=8.1 \mathrm{~Hz}), 7.27(\mathrm{~d}$, $2 \mathrm{H}, J=8.0 \mathrm{~Hz}), 4.12(\mathrm{~s}, 4 \mathrm{H}), 2.37(\mathrm{~s}, 3 \mathrm{H}), 2.19(\mathrm{t}, 4 \mathrm{H}, J=6.9 \mathrm{~Hz}), 1.47-1.39(\mathrm{~m}, 4 \mathrm{H})$, 1.39-1.29 (m, 4H), $0.86(\mathrm{t}, 6 \mathrm{H}, J=7.3 \mathrm{~Hz}) ;{ }^{13} \mathrm{C}$ NMR $\left(\mathrm{CDCl}_{3}, 125 \mathrm{MHz}\right): \delta 144.1$, 134.7, 129.6, 127.8, 80.9, 71.0, 67.8, 64.3, 37.2, 30.1, 21.9, 18.8, 13.5; HRMS (ESI) calcd for $\mathrm{C}_{25} \mathrm{H}_{30} \mathrm{NO}_{2} \mathrm{~S}[\mathrm{M}+\mathrm{H}]^{+} 408.1997$, found 408.1993 .

$453 \mathrm{mg}$, colorless oil. ${ }^{1} \mathrm{H}$ NMR ( $\left.\mathrm{CDCl}_{3}, 500 \mathrm{MHz}\right): \delta 8.06(\mathrm{~d}, 1 \mathrm{H}, J=7.6 \mathrm{~Hz}), 7.60$ (d, $1 \mathrm{H}, J=7.5 \mathrm{~Hz}), 7.50(\mathrm{t}, 1 \mathrm{H}, J=7.3 \mathrm{~Hz}), 7.46(\mathrm{~d}, 1 \mathrm{H}, J=7.5 \mathrm{~Hz}), 0.30(\mathrm{~s}, 9 \mathrm{H})$, 0.22 (s, 9H); ${ }^{13} \mathrm{C}$ NMR $\left(\mathrm{CDCl}_{3}, 125 \mathrm{MHz}\right): \delta$ 176.4, 139.2, 135.8, 132.6, 131.6, 128.9, 121.4, 101.8, 101.4, 93.0, 88.1, 80.3, 74.5, -0.4, -0.7; HRMS (ESI) calcd for $\mathrm{C}_{19} \mathrm{H}_{23} \mathrm{OSi}_{2}[\mathrm{M}+\mathrm{H}]^{+} 323.1287$, found 323.1288 . 


\section{Characterization Data for Products}

5aa: This product was isolated from stoichiometry screening of $\mathrm{PhNC}$ and $\mathrm{AcOH}$ and isolated in $18 \%$ yield<smiles>CC(=O)Oc1cc2c(c(C(C)(C)C)c1C(C)(C)C)CC(C(C)(C)C)(C(C)(C)C)C2</smiles>
(12 $\mathrm{mg}$, entry 7) as colorless oil after stirring the reaction mixture for $1.5 \mathrm{~h}$.

${ }^{1} \mathrm{H} \mathrm{NMR}\left(\mathrm{CDCl}_{3}, 500 \mathrm{MHz}\right): \delta 6.79(\mathrm{~s}, 1 \mathrm{H}), 3.76(\mathrm{~s}, 6 \mathrm{H}), 3.64(\mathrm{~s}, 2 \mathrm{H}), 3.61(\mathrm{~s}, 2 \mathrm{H})$, $2.26(\mathrm{~s}, 3 \mathrm{H}), 0.38(\mathrm{~s}, 9 \mathrm{H}), 0.24(\mathrm{~s}, 9 \mathrm{H}) . ;{ }^{13} \mathrm{C} \mathrm{NMR}\left(\mathrm{CDCl}_{3}, 125 \mathrm{MHz}\right): \delta$ 171.9, 170.0, 154.6, 142.0, 141.9, 131.3, 125.7, 118.7, 103.7, 103.3, 59.4, 53.1, 40.9, 40.6, 21.6, 1.0, -0.3. HRMS (ESI) calcd for $\mathrm{C}_{23} \mathrm{H}_{33} \mathrm{O}_{6} \mathrm{Si}_{2}[\mathrm{M}+\mathrm{H}]^{+} 461.1816$ found 461.1810.

7aa: This product was synthesized using procedure $\mathbf{A}$ and isolated in (62\% yield, $35.4 \mathrm{mg}$ ) as orange-yellow<smiles></smiles>
oil after stirring the reaction mixture for $1.5 \mathrm{~h} .{ }^{1} \mathbf{H}$ NMR $\left(\mathrm{CDCl}_{3}, 500 \mathrm{MHz}\right): \delta$ 7.45 (t, $2 \mathrm{H}, J=7.8 \mathrm{~Hz}), 7.39(\mathrm{t}, 1 \mathrm{H}, J=7.2 \mathrm{~Hz}), 7.22(\mathrm{~d}, 2 \mathrm{H}, J=7.3 \mathrm{~Hz}), 6.99$ (s, $1 \mathrm{H}), 3.76(\mathrm{~s}, 6 \mathrm{H}), 3.67(\mathrm{~s}, 2 \mathrm{H}), 3.59(\mathrm{~s}, 2 \mathrm{H}), 2.15(\mathrm{~s}, 3 \mathrm{H}), 0.42(\mathrm{~s}, 9 \mathrm{H}), 0.25(\mathrm{~s}$, $9 \mathrm{H}) ;{ }^{13} \mathrm{C}$ NMR $\left(\mathrm{CDCl}_{3}, 125 \mathrm{MHz}\right): \delta 173.2,172.4,171.9,144.6,143.6,139.6$, $139.4,138.8,129.8,128.9,128.8,126.4,120.4,104.0,103.9,59.2,53.1,41.0$, 41.0, 26.2, 0.9, -0.3; HRMS (ESI) calcd for $\mathrm{C}_{30} \mathrm{H}_{38} \mathrm{NO}_{6} \mathrm{Si}_{2}[\mathrm{M}+\mathrm{H}]^{+} 564.2238$ found 564.2239 .

7ab: This product was synthesized using procedure $\mathbf{A}$ and isolated in (57\% yield, $29.7 \mathrm{mg}$ ) as orangeyellow oil after stirring the reaction mixture for $1.5 \mathrm{~h} .{ }^{1} \mathrm{H} \mathbf{N M R}\left(\mathrm{CDCl}_{3}, 500 \mathrm{MHz}\right): \delta 7.48(\mathrm{t}, 2 \mathrm{H}, J=7.8 \mathrm{~Hz})$,<smiles>CCCC(=O)N(C(=O)c1cc2c(c(C(C)(C)C)c1C)CC(C(C)=O)(C(C)=O)C2)C(=O)C(C)(C)C</smiles>
$7.40(\mathrm{t}, 1 \mathrm{H}, J=7.3 \mathrm{~Hz}), 7.23(\mathrm{~d}, 2 \mathrm{H}, J=7.3 \mathrm{~Hz}), 6.98(\mathrm{~s}, 1 \mathrm{H}), 3.76(\mathrm{~s}, 6 \mathrm{H}), 3.68$ $(\mathrm{s}, 2 \mathrm{H}), 3.59(\mathrm{~s}, 2 \mathrm{H}), 2.29(\mathrm{t}, 2 \mathrm{H}, J=7.2 \mathrm{~Hz}), 1.60-1.52(\mathrm{~m}, 2 \mathrm{H}), 0.83(\mathrm{t}, 3 \mathrm{H}, J=$ $7.4 \mathrm{~Hz}), 0.42$ (s, 9H), 0.25 (s, 9H); ${ }^{13} \mathrm{C} \mathrm{NMR}\left(\mathrm{CDCl}_{3}, 125 \mathrm{MHz}\right): \delta 175.2,173.4$, $171.9,144.5,143.9,139.7,139.4,138.5,129.8,129.0,128.9,128.8,126.4$, 120.1, 104.0, 103.8, 59.3, 53.1, 41.0, 39.5, 18.0, 13.5, 0.9, -0.3; HRMS (ESI) calcd for $\mathrm{C}_{32} \mathrm{H}_{42} \mathrm{NO}_{6} \mathrm{Si}_{2}[\mathrm{M}+\mathrm{H}]^{+} 592.2551$ found 592.2554 .

7ac: This product was synthesized using procedure $\mathbf{A}$ and isolated in (63\% yield, $37.3 \mathrm{mg}$ ) as orange-red solid after stirring the reaction mixture for $1.5 \mathrm{~h} .{ }^{1} \mathrm{H} \mathrm{NMR}\left(\mathrm{CDCl}_{3}, 500 \mathrm{MHz}\right): \delta 7.47(\mathrm{t}, 2 \mathrm{H}, J=7.8 \mathrm{~Hz}), 7.40$<smiles>CC(=O)N(C(=O)c1cc2c(c(C(C)(C)C)c1C(C)(C)C)CC(C(C)=O)(C(C)(C)C)C2)c1ccccc1</smiles>
$(\mathrm{t}, 1 \mathrm{H}, J=7.4 \mathrm{~Hz}), 7.23(\mathrm{~d}, 2 \mathrm{H}, J=7.2 \mathrm{~Hz}), 6.97(\mathrm{~s}, 1 \mathrm{H}), 3.76(\mathrm{~s}, 6 \mathrm{H}), 3.68(\mathrm{~s}$, $2 \mathrm{H}), 3.59(\mathrm{~s}, 2 \mathrm{H}), 2.30(\mathrm{t}, 2 \mathrm{H}, J=7.4 \mathrm{~Hz}), 1.38-1.14(\mathrm{~m}, 30 \mathrm{H}), 0.87(\mathrm{t}, 3 \mathrm{H}, J$ $=6.9 \mathrm{~Hz}), 0.42(\mathrm{~s}, 9 \mathrm{H}), 0.25(\mathrm{~s}, 9 \mathrm{H}) ;{ }^{13} \mathrm{C}$ NMR $\left(\mathrm{CDCl}_{3}, 125 \mathrm{MHz}\right): \delta 175.4$, $173.4,171.9,144.5,143.9,139.6,139.5,138.5,129.8,128.8,126.4$, 120.1, 104.0, 103.8, 59.2, 53.1, 41.0, 41.0, 37.6, 31.9, 29.6, 29.4, 29.3, 29.0, 24.6, 22.7, 14.1, 0.9, -0.3; HRMS (ESI) calcd for $\mathrm{C}_{46} \mathrm{H}_{70} \mathrm{NO}_{6} \mathrm{Si}_{2}[\mathrm{M}+\mathrm{H}]^{+}$ 788.4742 found 788.4731 .

7ad: This product was synthesized using procedure $A$ and isolated in ( $50 \%$ yield, $32.4 \mathrm{mg}$ ) as orange-yellow

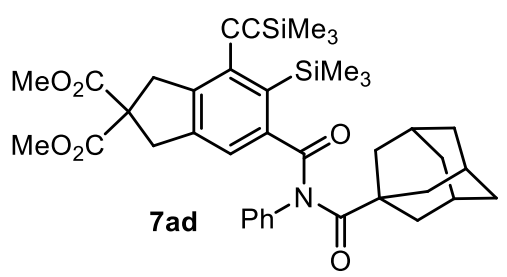
oil after stirring the reaction mixture for $1.5 \mathrm{~h} .{ }^{1} \mathbf{H}$ NMR $\left(\mathrm{CDCl}_{3}, 500\right.$ $\mathrm{MHz}): \delta 7.42(\mathrm{t}, 2 \mathrm{H}, J=7.8 \mathrm{~Hz}), 7.36(\mathrm{t}, 1 \mathrm{H}, J=7.3 \mathrm{~Hz}), 7.18(\mathrm{~d}, 2 \mathrm{H}, J=$ $7.4 \mathrm{~Hz}), 6.97(\mathrm{~s}, 1 \mathrm{H}), 3.76(\mathrm{~s}, 6 \mathrm{H}), 3.67(\mathrm{~s}, 2 \mathrm{H}), 3.58(\mathrm{~s}, 2 \mathrm{H}), 2.06-1.48$ $(\mathrm{m}, 15 \mathrm{H}), 0.40(\mathrm{~s}, 9 \mathrm{H}), 0.25(\mathrm{~s}, 9 \mathrm{H}) ;{ }^{13} \mathrm{C} \mathrm{NMR}\left(\mathrm{CDCl}_{3}, 125 \mathrm{MHz}\right): \delta 184.1$, $173.5,171.9,144.6,143.0,141.0,139.4,137.6,129.3,128.3,126.5$, 
120.6, 104.1, 103.8, 59.3, 53.1, 45.9, 41.0, 40.1, 36.2, 28.0, 1.0, -0.3; HRMS (ESI) calcd for $\mathrm{C}_{39} \mathrm{H}_{50} \mathrm{NO}_{6} \mathrm{Si}_{2}$ $[\mathrm{M}+\mathrm{H}]^{+} 684.3177$ found 684.3174 .

7ae: This product was synthesized using procedure $\mathbf{A}$ and isolated in (48\% yield, $26.5 \mathrm{mg}$ ) as white solid after stirring the reaction mixture for $1.5 \mathrm{~h} .{ }^{1} \mathrm{H}$ NMR $\left(\mathrm{CDCl}_{3}, 500 \mathrm{MHz}\right): \delta 7.65(\mathrm{~d}, 2 \mathrm{H}, J=8.0 \mathrm{~Hz}), 7.38(\mathrm{t}$,<smiles>CC(=O)C1(C(C)=O)Cc2cc(C(=O)N(C(=O)c3ccccc3)c3ccccc3)c(C(C)(C)C)c(C(C)(C)C)c2C1</smiles>
$1 \mathrm{H}, J=6.9 \mathrm{~Hz}), 7.34(\mathrm{t}, 2 \mathrm{H}, J=7.8 \mathrm{~Hz}), 7.28(\mathrm{~d}, 2 \mathrm{H}, J=7.5 \mathrm{~Hz}), 7.24(\mathrm{t}, 1 \mathrm{H}, J=$ $7.1 \mathrm{~Hz}), 7.20(\mathrm{~s}, 1 \mathrm{H}), 7.18(\mathrm{~d}, 2 \mathrm{H}, J=8.2 \mathrm{~Hz}), 3.75(\mathrm{~s}, 6 \mathrm{H}), 3.67(\mathrm{~s}, 2 \mathrm{H}), 3.55(\mathrm{~s}$, $2 \mathrm{H}), 0.45(\mathrm{~s}, 9 \mathrm{H}), 0.26(\mathrm{~s}, 9 \mathrm{H}) ;{ }^{13} \mathrm{C}$ NMR $\left(\mathrm{CDCl}_{3}, 125 \mathrm{MHz}\right): \delta 173.8,172.0$, $171.9,145.1,143.4,141.4,139.8,139.2,133.7,132.5,130.0,129.5,128.4$, 127.9, 127.8, 126.8, 120.1, 104.0, 103.9, 59.2, 53.1, 41.0, 1.1, -0.3; HRMS (ESI) calcd for $\mathrm{C}_{35} \mathrm{H}_{40} \mathrm{NO}_{6} \mathrm{Si}_{2}[\mathrm{M}+\mathrm{H}]^{+} 626.2394$ found 626.2399 .

7af: This product was synthesized using procedure $\mathbf{A}$ and isolated in (46\% yield, $22.8 \mathrm{mg}$ ) as orange-yellow<smiles>CCCCCC(C)(C)OC(C)=O</smiles>
oil after stirring the reaction mixture for $1.5 \mathrm{~h} .{ }^{1} \mathrm{H} \mathrm{NMR}\left(\mathrm{CDCl}_{3}, 500 \mathrm{MHz}\right): \delta 7.12$ $(\mathrm{d}, 2 \mathrm{H}, J=8.9 \mathrm{~Hz}), 6.96(\mathrm{~s}, 1 \mathrm{H}), 6.94(\mathrm{~d}, 2 \mathrm{H}, J=8.9 \mathrm{~Hz}), 3.82(\mathrm{~s}, 3 \mathrm{H}), 3.76(\mathrm{~s}, 6 \mathrm{H})$, $3.66(\mathrm{~s}, 2 \mathrm{H}), 3.58(\mathrm{~s}, 2 \mathrm{H}), 2.17(\mathrm{~s}, 3 \mathrm{H}), 0.42(\mathrm{~s}, 9 \mathrm{H}), 0.25(\mathrm{~s}, 9 \mathrm{H}) ;{ }^{13} \mathrm{C} \mathrm{NMR}\left(\mathrm{CDCl}_{3}\right.$, $125 \mathrm{MHz}$ ): $\delta$ 173.4, 172.8, 171.9, 159.6, 144.5, 143.6, 139.6, 139.3, 131.3, 129.7, $126.3,120.4,115.0,104.0,103.8,59.2,55.5,53.1,41.0,26.2,0.9,-0.3$; HRMS (ESI) calcd for $\mathrm{C}_{31} \mathrm{H}_{40} \mathrm{NO}_{7} \mathrm{Si}_{2}[\mathrm{M}+\mathrm{H}]^{+} 594.2343$ found 594.2353 .

7ag: This product was synthesized using procedure $\mathbf{A}$ and isolated in (75\% yield, $38.0 \mathrm{mg}$ ) as light brown<smiles>CC(=O)N(C(C)=O)c1ccc(Br)cc1C(=O)N(CC(C)(C)C)c1ccc2c(c1C(C)(C)C)CC(C)(C(C)=O)C2</smiles>
solid after stirring the reaction mixture for $1.5 \mathrm{~h} .{ }^{1} \mathrm{H} \mathrm{NMR}\left(\mathrm{CDCl}_{3}, 500 \mathrm{MHz}\right): \delta$ $8.56(\mathrm{~d}, 2 \mathrm{H}, J=8.6 \mathrm{~Hz}), 7.08(\mathrm{~d}, 2 \mathrm{H}, J=8.6 \mathrm{~Hz}), 6.95(\mathrm{~s}, 1 \mathrm{H}), 3.76(\mathrm{~s}, 6 \mathrm{H}), 3.66(\mathrm{~s}$, $2 \mathrm{H}), 3.58(\mathrm{~s}, 2 \mathrm{H}), 2.20(\mathrm{~s}, 3 \mathrm{H}), 0.41(\mathrm{~s}, 9 \mathrm{H}), 0.25(\mathrm{~s}, 9 \mathrm{H}) ;{ }^{13} \mathrm{C}$ NMR $\left(\mathrm{CDCl}_{3}, 125\right.$ $\mathrm{MHz}): \delta 172.9,172.1,171.8,144.9,143.0,139.8,139.4,137.8,133.0,130.5$, $126.5,122.9,120.5,104.2,103.8,59.2,53.2,41.0,40.9,26.3,0.9,-0.3$; HRMS (ESI) calcd for $\mathrm{C}_{30} \mathrm{H}_{37} \mathrm{NO}_{6} \mathrm{Si}{ }_{2} \mathrm{Br}[\mathrm{M}+\mathrm{H}]^{+} 642.1343$ found 642.1342 .

7ah: This product was synthesized using procedure $\mathbf{A}$ and isolated in (63\% yield, $27.0 \mathrm{mg}$ ) as white solid<smiles></smiles>
after stirring the reaction mixture for $1.5 \mathrm{~h} .{ }^{1} \mathbf{H} \mathbf{N M R}\left(\mathrm{CDCl}_{3}, 500 \mathrm{MHz}\right): \delta 8.10$ $(\mathrm{d}, 2 \mathrm{H}, J=8.4 \mathrm{~Hz}), 7.28(\mathrm{~d}, 2 \mathrm{H}, J=8.4 \mathrm{~Hz}), 6.96(\mathrm{~s}, 1 \mathrm{H}), 3.93(\mathrm{~s}, 3 \mathrm{H}), 3.76(\mathrm{~s}, 6 \mathrm{H})$, $3.65(\mathrm{~s}, 2 \mathrm{H}), 3.56(\mathrm{~s}, 2 \mathrm{H}), 2.23(\mathrm{~s}, 3 \mathrm{H}), 0.43(\mathrm{~s}, 9 \mathrm{H}), 0.25(\mathrm{~s}, 9 \mathrm{H}) ;{ }^{13} \mathrm{C} \mathrm{NMR}\left(\mathrm{CDCl}_{3}\right.$, $125 \mathrm{MHz}$ ): $\delta$ 172.8, 172.1, 171.8, 166.0, 145.0, 142.8, 139.8, 139.5, 131.0, 130.5, 129.0, 126.5, 120.7, 104.3, 103.8, 59.2, 53.1, 52.4, 41.0, 40.9, 26.4, 1.0, -0.3; HRMS (ESI) calcd for $\mathrm{C}_{32} \mathrm{H}_{40} \mathrm{NO}_{8} \mathrm{Si}_{2}[\mathrm{M}+\mathrm{H}]^{+} 622.2292$ found 622.2294 . 
7ai: This product was synthesized using procedure $A$ and isolated in ( $60 \%$ yield, $28.3 \mathrm{mg}$ ) as orange-yellow<smiles>CC(=O)N(C)C(=O)c1cc([N+](=O)[O-])ccc1N(C(C)=O)C(C)=O</smiles>
solid after stirring the reaction mixture for $1.5 \mathrm{~h} .{ }^{1} \mathrm{H} \mathrm{NMR} \delta\left(\mathrm{CDCl}_{3}, 500 \mathrm{MHz}\right): \delta$ $8.27(\mathrm{~d}, 2 \mathrm{H}, J=8.8 \mathrm{~Hz}), 7.36(\mathrm{~d}, 2 \mathrm{H}, J=8.8 \mathrm{~Hz}), 6.94(\mathrm{~s}, 1 \mathrm{H}), 3.76(\mathrm{~s}, 6 \mathrm{H}), 3.64(\mathrm{~s}$, $2 \mathrm{H}), 3.54(\mathrm{~s}, 2 \mathrm{H}), 2.32(\mathrm{~s}, 3 \mathrm{H}), 0.44(\mathrm{~s}, 9 \mathrm{H}), 0.26(\mathrm{~s}, 9 \mathrm{H}) ;{ }^{13} \mathrm{C} \mathrm{NMR}\left(\mathrm{CDCl}_{3}, 125\right.$ $\mathrm{MHz}): \delta 172.4,171.9,171.7,147.5,145.5,144.6,142.1,140.1,139.5,130.1$, $126.8,124.8,121.0,104.8,103.5,59.1,53.2,41.0,40.8,26.7,1.0,-0.3$; HRMS (ESI) calcd for $\mathrm{C}_{30} \mathrm{H}_{37} \mathrm{~N}_{2} \mathrm{O}_{8} \mathrm{Si}_{2}[\mathrm{M}+\mathrm{H}]^{+} 609.2088$ found 609.2081.

7aj: This product was synthesized using procedure $\mathbf{A}$ and isolated in ( $80 \%$ yield, $39.4 \mathrm{mg}$ ) as orange solid after stirring the reaction mixture for $1.5 \mathrm{~h} .{ }^{1} \mathrm{H}$ NMR $\left(\mathrm{CDCl}_{3}, 500 \mathrm{MHz}\right): \delta 8.74$<smiles>CC(=O)N(C(C)=O)c1cnc2ccccc2c1</smiles>

7aj (d, $1 \mathrm{H}, J=2.4 \mathrm{~Hz}$ ), $8.13(\mathrm{~d}, 1 \mathrm{H}, J=8.4 \mathrm{~Hz}), 7.97(\mathrm{~d}, 1 \mathrm{H}, J=2.2 \mathrm{~Hz}), 7.80(\mathrm{~d}, 1 \mathrm{H}, J$ $=8.3 \mathrm{~Hz}), 7.77(\mathrm{td}, 2 \mathrm{H}, J=7.7 \mathrm{~Hz}, J=1.2 \mathrm{~Hz}), 7.61(\mathrm{t}, 1 \mathrm{H}, J=7.8 \mathrm{~Hz}), 7.04(\mathrm{~s}, 1 \mathrm{H})$, $3.73(\mathrm{~s}, 6 \mathrm{H}), 3.62(\mathrm{~s}, 2 \mathrm{H}), 3.56(\mathrm{~s}, 2 \mathrm{H}), 2.34(\mathrm{~s}, 3 \mathrm{H}), 0.47(\mathrm{~s}, 9 \mathrm{H}), 0.24(\mathrm{~s}, 9 \mathrm{H}) ;{ }^{13} \mathrm{C}$ NMR $\left(\mathrm{CDCl}_{3}, 125 \mathrm{MHz}\right): \delta$ 173.0, 172.4, 171.7, 150.3, 147.3, 145.3, 142.5, $140.0,139.5,135.3,132.3,130.6,129.5,127.9,127.7,127.6,126.7,120.8$, 104.5, 103.6, 59.1, 53.1, 41.0, 40.9, 26.7, 1.0, -0.3; HRMS (ESI) calcd for $\mathrm{C}_{33} \mathrm{H}_{39} \mathrm{~N}_{2} \mathrm{O}_{6} \mathrm{Si}_{2}[\mathrm{M}+\mathrm{H}]^{+} 615.2347$ found 615.2352 .

7ak: This product was synthesized using procedure $A$ and isolated in (64\% yield, $31.8 \mathrm{mg}$ ) as yellow-green solid after stirring the reaction mixture for $1.5 \mathrm{~h} .{ }^{1} \mathrm{H}$ NMR $\left(\mathrm{CDCl}_{3}, 500 \mathrm{MHz}\right): \delta 7.70(\mathrm{~d}, 1 \mathrm{H}, J=15.5 \mathrm{~Hz})$,<smiles>COC(=O)C1(C(C)(C)C)Cc2cc(C(=O)N(C(=O)/C=C/c3ccccc3)c3ccccc3)c(C(C)(C)C)c(C(C)(C)C)c2C1</smiles>
$7.52(\mathrm{t}, 2 \mathrm{H}, \mathrm{J}=7.8 \mathrm{~Hz}), 7.49-7.43(\mathrm{~m}, 1 \mathrm{H}), 7.38-7.27(\mathrm{~m}, 7 \mathrm{H}), 7.09(\mathrm{~s}, 1 \mathrm{H})$, $6.37(\mathrm{~d}, 1 \mathrm{H}, J=15.5 \mathrm{~Hz}), 3.75(\mathrm{~s}, 6 \mathrm{H}), 3.70(\mathrm{~s}, 2 \mathrm{H}), 3.61(\mathrm{~s}, 2 \mathrm{H}), 0.45(\mathrm{~s}$, 9H), $0.27(\mathrm{~s}, 9 \mathrm{H}) ;{ }^{13} \mathrm{C} \mathrm{NMR}\left(\mathrm{CDCl}_{3}, 125 \mathrm{MHz}\right): \delta 173.7,171.9,167.6,146.0$, $144.7,143.9,140.1,139.8,138.0,134.2,130.7,129.9,128.9,128.7$, $128.4,126.5,120.1,119.1,104.1,103.7,59.2,53.1,41.1,41.0,0.9,-0.3$; HRMS (ESI) calcd for $\mathrm{C}_{37} \mathrm{H}_{42} \mathrm{NO}_{6} \mathrm{Si}_{2}[\mathrm{M}+\mathrm{H}]^{+} 652.2551$ found 652.2554 .

7ba: This product was synthesized using procedure $A$ and isolated in ( $52 \%$ yield, $18.0 \mathrm{mg}$ ) as orange-<smiles>CC(=O)c1c2c(cc(N(C(C)=O)C(C)=O)c1C(=O)N(c1ccccc1)c1ccccc1)CN([Te])C2</smilesyellow solid after stirring the reaction mixture for $30 \mathrm{~min} .{ }^{1}{ }^{\mathrm{H}} \mathbf{~ N M R}\left(\mathrm{CDCl}_{3}, 500 \mathrm{MHz}\right)$ : $\delta 7.76(\mathrm{~d}, 2 \mathrm{H}, J=8.1 \mathrm{~Hz}), 7.48(\mathrm{t}, 2 \mathrm{H}, J=7.6 \mathrm{~Hz}), 7.42(\mathrm{t}, 1 \mathrm{H}, J=7.3 \mathrm{~Hz}), 7.33(\mathrm{~d}, 2 \mathrm{H}, J$ $=8.0 \mathrm{~Hz}), 7.22(\mathrm{~d}, 2 \mathrm{H}, J=7.8 \mathrm{~Hz}), 6.94(\mathrm{~s}, 1 \mathrm{H}), 4.63(\mathrm{~s}, 2 \mathrm{H}), 4.61(\mathrm{~s}, 2 \mathrm{H}), 2.42(\mathrm{~s}, 3 \mathrm{H})$, $2.06(\mathrm{~s}, 3 \mathrm{H}), 0.40(\mathrm{~s}, 9 \mathrm{H}), 0.26(\mathrm{~s}, 9 \mathrm{H}) ;{ }^{13} \mathrm{C}$ NMR $\left(\mathrm{CDCl}_{3}, 125 \mathrm{MHz}\right): \delta$ 172.7, 172.3, $144.6,143.9,140.7,140.4,138.5,135.9,133.6,130.0,129.2,128.6,127.5,125.2$, 118.1, 105.1, 102.7, 54.3, 54.2, 25.9, 21.5, 0.7, -0.4; HRMS (ESI) calcd for $\mathrm{C}_{32} \mathrm{H}_{39} \mathrm{~N}_{2} \mathrm{O}_{4} \mathrm{Si}_{2} \mathrm{~S}[\mathrm{M}+\mathrm{H}]^{+} 603.2169$ found 603.2174 .

7ca: This product was synthesized using procedure $A$ and isolated in (62\% yield, $28.4 \mathrm{mg}$ ) yellow-green<smiles>CC(=O)N(C(=O)c1cc2c(c(C(C)(C)C)c1C)CC1(C2)c2ccccc2-c2ccccc21)c1ccccc1</smiles>
solid after stirring the reaction mixture for 5 h. ${ }^{1} \mathbf{H ~ N M R ~}\left(\mathrm{CDCl}_{3}, 500 \mathrm{MHz}\right): \delta 7.74$ $(\mathrm{d}, 2 \mathrm{H}, J=7.6 \mathrm{~Hz}), 7.48-7.40(\mathrm{~m}, 3 \mathrm{H}), 7.37(\mathrm{t}, 2 \mathrm{H}, J=7.5 \mathrm{~Hz}), 7.29-7.21(\mathrm{~m}, 4 \mathrm{H})$, 7.21-7.10 (m, 2H), $7.07(\mathrm{~s}, 1 \mathrm{H}), 3.47(\mathrm{~s}, 2 \mathrm{H}), 3.37(\mathrm{~s}, 2 \mathrm{H}), 2.30(\mathrm{~s}, 3 \mathrm{H}), 0.50(\mathrm{~s}, 9 \mathrm{H})$, $0.15(\mathrm{~s}, 9 \mathrm{H}) ;{ }^{13} \mathrm{C}$ NMR $\left(\mathrm{CDCl}_{3}, 125 \mathrm{MHz}\right): \delta 173.5,172.7,152.2,147.6,143.1$, $142.5,139.5,139.0,138.8,129.7,129.0,128.7,127.7,127.5,126.6,122.5,121.2$, 
119.8, 104.2, 103.6, 56.3, 45.8, 45.5, 26.6, 1.1, -0.4; HRMS (ESI) calcd for $\mathrm{C}_{38} \mathrm{H}_{40} \mathrm{NO}_{2} \mathrm{Si}_{2}[\mathrm{M}+\mathrm{H}]^{+} 598.2598$ found 598.2600 .

7da: This product was synthesized using procedure $A$ and isolated in (43\% yield, $17.8 \mathrm{mg}$ ) as orange-yellow<smiles>CCCc1cc2c(cc1C(=O)N(C(C)=O)c1ccccc1)CN(c1ccccc1)C2=O</smiles>
oil after stirring the reaction mixture for $2 \mathrm{~h} .{ }^{1} \mathrm{H} ~ \mathrm{NMR}\left(\mathrm{CDCl}_{3}, 500 \mathrm{MHz}\right): \delta 8.16(\mathrm{~s}$, $1 \mathrm{H}), 7.83(\mathrm{~d}, 2 \mathrm{H}, J=8.1 \mathrm{~Hz}), 7.54-7.47(\mathrm{~m}, 3 \mathrm{H}), 7.44(\mathrm{t}, 3 \mathrm{H}, J=7.6 \mathrm{~Hz}), 7.28(\mathrm{~d}, 2 \mathrm{H}$, $J=7.8 \mathrm{~Hz}), 7.20(\mathrm{t}, 1 \mathrm{H}, J=7.4 \mathrm{~Hz}), 4.84(\mathrm{~s}, 2 \mathrm{H}), 2.15(\mathrm{~s}, 3 \mathrm{H}), 1.00-0.88(\mathrm{~m}, 15 \mathrm{H}) ;{ }^{13} \mathrm{C}$ NMR $\left(\mathrm{CDCl}_{3}, 125 \mathrm{MHz}\right): \delta 173.3,172.4,166.9,146.6,139.8,139.4,138.8,138.0$, 133.3, 132.0, 130.0, 129.2, 129.1, 128.7, 124.7, 120.2, 119.7, 50.6, 26.0, 7.5, 3.9; HRMS (ESI) calcd for $\mathrm{C}_{29} \mathrm{H}_{33} \mathrm{~N}_{2} \mathrm{O}_{3} \mathrm{Si}[\mathrm{M}+\mathrm{H}]^{+} 485.2260$ found 485.2259 .

7ea: This product was synthesized using procedure $\mathbf{A}$ and isolated in (74\% yield, $26.7 \mathrm{mg}$ ) as orange-red<smiles>CCOC(=O)C1(C(=O)OCC)Cc2c(C(=O)N(C(C)=O)c3ccccc3)cc(C(C)(C)C)c(C(C)(C)C)c2C1</smiles>
oil after stirring the reaction mixture for $2 \mathrm{~h} .{ }^{1} \mathrm{H} \mathrm{NMR}\left(\mathrm{CDCl}_{3}, 500 \mathrm{MHz}\right): \delta 7.55-$ $7.39(\mathrm{~m}, 3 \mathrm{H}), 7.25(\mathrm{~d}, 1 \mathrm{H}, J=7.7 \mathrm{~Hz}), 6.79(\mathrm{~s}, 1 \mathrm{H}), 4.22(\mathrm{q}, 2 \mathrm{H}, J=7.1 \mathrm{~Hz}), 4.22(\mathrm{q}$, $2 \mathrm{H}, J=7.0 \mathrm{~Hz}), 3.64(\mathrm{~s}, 2 \mathrm{H}), 3.59(\mathrm{~s}, 2 \mathrm{H}), 2.02(\mathrm{br}, 3 \mathrm{H}), 1.61(\mathrm{~s}, 9 \mathrm{H}), 1.34(\mathrm{~s}, 9 \mathrm{H})$, $1.26(\mathrm{t}, 6 \mathrm{H}, J=7.1 \mathrm{~Hz}) ;{ }^{13} \mathrm{C} \mathrm{NMR}\left(\mathrm{CDCl}_{3}, 125 \mathrm{MHz}\right): \delta 173.8,171.6,145.8,145.6$, $138.9,136.2,129.8,129.0,128.7,128.5,121.0,119.8,109.9,78.9,61.8,59.4,41.7$, 40.7, 30.7, 30.6, 14.0; HRMS (ESI) calcd for $\mathrm{C}_{34} \mathrm{H}_{42} \mathrm{NO}_{6}[\mathrm{M}+\mathrm{H}]^{+} 560.3012$ found 560.3020 .

8aa: This product was synthesized using procedure $\mathbf{B}$ and isolated in (78\% yield, $30.6 \mathrm{mg}$ ) as yellow oil after stirring the reaction mixture for $1.5 \mathrm{~h} .{ }^{1} \mathrm{H}$ NMR $\left(\mathrm{CDCl}_{3}, 500 \mathrm{MHz}\right): \delta 7.10(\mathrm{t}, 2 \mathrm{H}, J=7.5 \mathrm{~Hz}), 6.88(\mathrm{t}$, $1 \mathrm{H}, J=7.2 \mathrm{~Hz}), 6.79(\mathrm{~s}, 1 \mathrm{H}), 6.69(\mathrm{~d}, 2 \mathrm{H}, J=7.6 \mathrm{~Hz}), 4.32(\mathrm{br}, 2 \mathrm{H}), 3.73(\mathrm{~s}, 6 \mathrm{H}), 3.61(\mathrm{~s}, 2 \mathrm{H}), 3.48(\mathrm{~s}, 2 \mathrm{H})$,

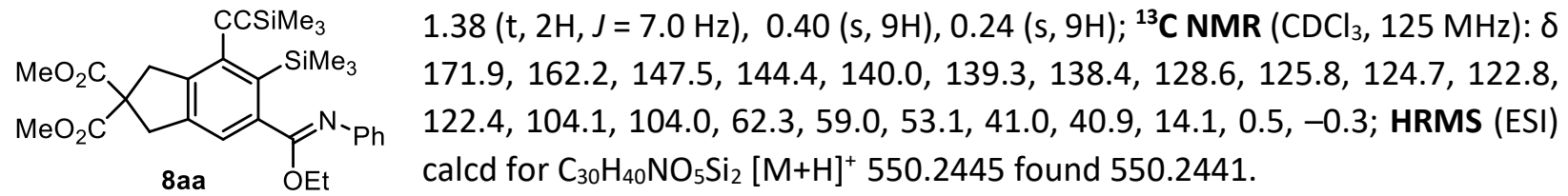

8ab: This product was synthesized using procedure $\mathbf{B}$ and isolated in (61\% yield, $26.1 \mathrm{mg}$ ) as light yellow solid after stirring the reaction mixture for $1.5 \mathrm{~h} .{ }^{1} \mathrm{H} \mathrm{NMR}\left(\mathrm{CDCl}_{3}, 500 \mathrm{MHz}\right): \delta 7.13(\mathrm{t}, 2 \mathrm{H}, J=7.6 \mathrm{~Hz}), 6.95$ $(\mathrm{t}, 1 \mathrm{H}, J=7.0 \mathrm{~Hz}), 6.82(\mathrm{~s}, 1 \mathrm{H}), 6.69(\mathrm{~d}, 2 \mathrm{H}, J=7.6 \mathrm{~Hz}), 4.83(\mathrm{br}, 1 \mathrm{H}), 4.50(\mathrm{br}, 1 \mathrm{H}), 3.75(\mathrm{~s}, 6 \mathrm{H}), 3.64(\mathrm{~s}, 2 \mathrm{H})$,

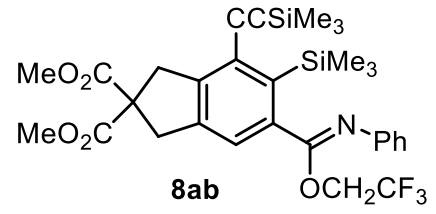
$3.51(\mathrm{~s}, 2 \mathrm{H}), 0.36(\mathrm{~s}, 9 \mathrm{H}), 0.24(\mathrm{~s}, 9 \mathrm{H}) ;{ }^{13} \mathrm{C} \mathrm{NMR}\left(\mathrm{CDCl}_{3}, 125 \mathrm{MHz}\right): \delta 171.9$, $160.1,145.7,145.1,140.3,139.5,136.4,128.8,126.1,124.7,123.8,123.6$ $(q, J=277.1 \mathrm{~Hz}), 122.3,104.4,103.8,62.8(\mathrm{q}, J=35.7 \mathrm{~Hz}), 58.9,53.1,41.0$, 40.9, $-0.3 ;{ }^{19} \mathrm{~F} \mathrm{NMR}\left(375 \mathrm{MHz}, \mathrm{CDCl}_{3}\right): \delta-73.3 \mathrm{~Hz}(\mathrm{t}, J=8.1 \mathrm{~Hz})$; HRMS (ESI) calcd for $\mathrm{C}_{30} \mathrm{H}_{37} \mathrm{NO}_{5} \mathrm{~F}_{3} \mathrm{Si}_{2}[\mathrm{M}+\mathrm{H}]^{+} 604.2162$ found 604.2166 .

8ac: This product was synthesized using procedure $\mathbf{B}$ and isolated in (90\% yield, $39.4 \mathrm{mg}$ ) as yellow solid after stirring the reaction mixture for $1.5 \mathrm{~h} .{ }^{1} \mathrm{H}$ NMR $\left(\mathrm{CDCl}_{3}, 500 \mathrm{MHz}\right): \delta 7.48-7.34(\mathrm{~m}, 4 \mathrm{H}), 7.20(\mathrm{br}, 1 \mathrm{H})$,

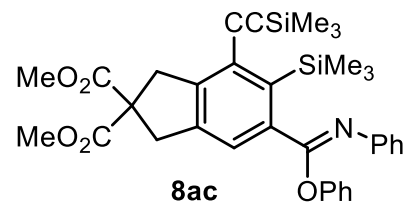
7.13-6.99 (m, 4H), 6.94-6.84 (m, 1H), $6.66(\mathrm{~d}, 2 \mathrm{H}, J=6.6 \mathrm{~Hz}), 3.76(\mathrm{~s}, 6 \mathrm{H}), 3.67$ $(\mathrm{s}, 2 \mathrm{H}), 3.58(\mathrm{~s}, 2 \mathrm{H}), 0.44(\mathrm{~s}, 9 \mathrm{H}), 0.25(\mathrm{~s}, 9 \mathrm{H}) ;{ }^{13} \mathrm{C} \mathrm{NMR}\left(\mathrm{CDCl}_{3}, 125 \mathrm{MHz}\right): \delta$ $171.9,160.9,153.0,146.2,144.8,140.3,139.2,137.7,129.2,128.5,126.0$, $124.9,123.4,122.3,122.0,104.2,104.0,59.1,53.1,41.1,40.9,0.7,-0.3$; HRMS (ESI) calcd for $\mathrm{C}_{34} \mathrm{H}_{40} \mathrm{NO}_{5} \mathrm{Si}_{2}[\mathrm{M}+\mathrm{H}]^{+} 598.2445$ found 598.2466 
8ad: This product was synthesized using procedure $\mathbf{B}$ and isolated in (67\% yield, $29.2 \mathrm{mg}$ ) as light yellow oil after stirring the reaction mixture for $1.5 \mathrm{~h} .{ }^{1} \mathrm{H} \mathrm{NMR}\left(\mathrm{CDCl}_{3}, 500 \mathrm{MHz}\right): \delta 7.10(\mathrm{t}, 2 \mathrm{H}, J=7.3 \mathrm{~Hz}), 6.89(\mathrm{t}$, $1 \mathrm{H}, J=7.0 \mathrm{~Hz}), 6.79(\mathrm{~s}, 1 \mathrm{H}), 6.69(\mathrm{~d}, 2 \mathrm{H}, J=7.4 \mathrm{~Hz}), 6.14-6.01(\mathrm{~m}, 1 \mathrm{H}), 5.40$  (d, $1 \mathrm{H}, J=17.1 \mathrm{~Hz}), 5.26(\mathrm{~d}, 1 \mathrm{H}, J=10.4 \mathrm{~Hz}), 4.80(\mathrm{br}, 2 \mathrm{H}), 3.73(\mathrm{~s}, 6 \mathrm{H}), 3.62(\mathrm{~s}$, $2 \mathrm{H}), 3.48(\mathrm{~s}, 2 \mathrm{H}), 0.39(\mathrm{~s}, 9 \mathrm{H}), 0.24(\mathrm{~s}, 9 \mathrm{H}) ;{ }^{13} \mathrm{C} \mathrm{NMR}\left(\mathrm{CDCl}_{3}, 125 \mathrm{MHz}\right): \delta 171.9$, $161.7,147.2$, 144.5, 140.0, 139.4, 138.0, 132.7, 128.6, 125.9, 124.7, 123.0, $122.4,118.1,104.1,104.0,67.3,59.0,53.1,41.0,40.9,0.5,-0.3$; HRMS (ESI) calcd for $\mathrm{C}_{31} \mathrm{H}_{40} \mathrm{NO}_{5} \mathrm{Si}_{2}[\mathrm{M}+\mathrm{H}]^{+} 562.2445$ found 562.2468 .

8ae: This product was synthesized using procedure $\mathbf{B}$ and isolated in (63\% yield, $25.5 \mathrm{mg}$ ) as orange-yellow<smiles>C/C=C/CO/C(=N/c1ccccc1)c1cc2c(c(C(C)(C)C)c1C(C)(C)C)CC(C(C)=O)(C(C)=O)C2</smiles>
oil after stirring the reaction mixture for $1.5 \mathrm{~h} .{ }^{1} \mathbf{H}$ NMR $\left(\mathrm{CDCl}_{3}, 500 \mathrm{MHz}\right): \delta$ 7.15-7.05 (m, 2H), 6.92-6.85 (m, 1H), $6.80(\mathrm{~s}, 1 \mathrm{H}), 6.69(\mathrm{~d}, 2 \mathrm{H}, \mathrm{J}=7.2 \mathrm{~Hz}), 5.92-$ $5.80(\mathrm{~m}, 1 \mathrm{H}), 5.79-5.67(\mathrm{~m}, 1 \mathrm{H}), 4.72(\mathrm{br}, 2 \mathrm{H}), 3.73(\mathrm{~s}, 6 \mathrm{H}), 3.61(\mathrm{~s}, 2 \mathrm{H}), 3.48(\mathrm{~s}$, $2 \mathrm{H}), 0.39(\mathrm{~s}, 9 \mathrm{H}), 0.24(\mathrm{~s}, 9 \mathrm{H}) ;{ }^{13} \mathrm{C} \mathrm{NMR}\left(\mathrm{CDCl}_{3}, 125 \mathrm{MHz}\right): \delta 171.9,161.8,147.3$, $144.5,140.0,139.3,138.2,131.1,128.6,125.8,125.6,124.7,122.9,122.4$, 104.1, 103.9, 67.4, 59.0, 53.1, 41.0, 40.9, 17.9, 0.5, -0.3; HRMS (ESI) calcd for $\mathrm{C}_{32} \mathrm{H}_{42} \mathrm{NO}_{5} \mathrm{Si}_{2}[\mathrm{M}+\mathrm{H}]^{+} 576.2602$ found 576.2585 .

8af: This product was synthesized using procedure B with some modification and isolated in (72\% yield, $35.4 \mathrm{mg}$ ) as orange yellow oil after stirring the reaction mixture for $1.5 \mathrm{~h}$. Phenyl isocyanide $(0.4 \mathrm{mmol})$ and alcohol $(0.2 \mathrm{mmol})$ was used here to avoid possible ene-reaction between aryne and prenyl alcohol. The purification was performed using neutral alumina column chromatography. ${ }^{1} \mathbf{H}$ NMR $\left(\mathrm{CDCl}_{3}, 500\right.$<smiles>CC(=O)C1(C(C)=O)Cc2cc(/C(=N/c3ccccc3)OC/C=C(/C)Br)c(C(C)(C)C)c(C(C)(C)C)c2C1</smiles>
$\mathrm{MHz}): \delta 7.09(\mathrm{t}, 2 \mathrm{H}, J=7.4 \mathrm{~Hz}), 6.89(\mathrm{t}, 1 \mathrm{H}, J=7.0 \mathrm{~Hz}), 6.81(\mathrm{~s}, 1 \mathrm{H}), 6.70(\mathrm{~d}, 2 \mathrm{H}$, $J=7.5 \mathrm{~Hz}), 5.53-5.43(\mathrm{~m}, 1 \mathrm{H}), 4.80(\mathrm{br}, 2 \mathrm{H}), 3.73(\mathrm{~s}, 6 \mathrm{H}), 3.60(\mathrm{~s}, 2 \mathrm{H}), 3.48(\mathrm{~s}$, $2 \mathrm{H}), 1.78(\mathrm{~s}, 3 \mathrm{H}), 1.76(\mathrm{~s}, 3 \mathrm{H}) 0.38(\mathrm{~s}, 9 \mathrm{H}), 0.23(\mathrm{~s}, 9 \mathrm{H}) ;{ }^{13} \mathrm{C}$ NMR $\left(\mathrm{CDCl}_{3}, 125\right.$ $\mathrm{MHz}): \delta 171.9,162.1,147.3,144.4,139.9,139.3,138.3,128.6,125.8,124.8$, $122.9,122.4,119.1,104.1,103.9,63.6,59.1,53.1,41.0,40.9,25.9,18.2,0.5$, -0.3 ; HRMS (ESI) calcd for $\mathrm{C}_{33} \mathrm{H}_{43} \mathrm{NO}_{5} \mathrm{NaSi}_{2}[\mathrm{M}+\mathrm{Na}]^{+} 612.2578$ found 612.2557 .

8ag: This product was synthesized using procedure $\mathbf{B}$ and isolated in ( $81 \%$ yield, $44.2 \mathrm{mg}$ ) as orange yellow solid after stirring the reaction mixture for $1.5 \mathrm{~h} .{ }^{1} \mathrm{H} \mathrm{NMR}\left(\mathrm{CDCl}_{3}, 500 \mathrm{MHz}\right): \delta 8.00(\mathrm{~d}, 2 \mathrm{H}, J=7.9 \mathrm{~Hz}), 7.49-$

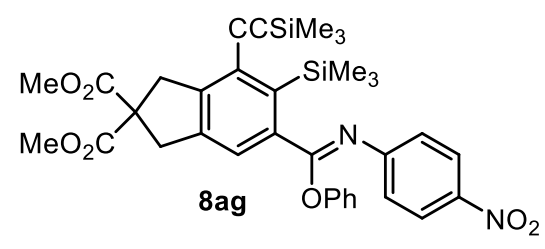
$7.30(\mathrm{~m}, 4 \mathrm{H}), 7.26-7.20(\mathrm{~m}, 1 \mathrm{H}), 7.05(\mathrm{~s}, 1 \mathrm{H}), 6.83-6.69(\mathrm{~m}, 2 \mathrm{H}), 3.77$ $(\mathrm{s}, 6 \mathrm{H}), 3.67(\mathrm{~s}, 2 \mathrm{H}), 3.59(\mathrm{~s}, 2 \mathrm{H}), 0.46(\mathrm{~s}, 9 \mathrm{H}), 0.25(\mathrm{~s}, 9 \mathrm{H}) ;{ }^{13} \mathrm{C}$ NMR $\left(\mathrm{CDCl}_{3}, 125 \mathrm{MHz}\right): \delta 171.8,152.9,152.7,145.6,143.6,140.6,139.1$, $136.6,129.4,126.5,125.6,124.6,122.4,121.7,105.0,103.5,59.0$, $53.2,41.0,40.9,0.9,-0.4$; HRMS (ESI) calcd for $\mathrm{C}_{34} \mathrm{H}_{39} \mathrm{~N}_{2} \mathrm{O}_{7} \mathrm{Si}_{2}[\mathrm{M}+\mathrm{H}]^{+}$ 643.2296 found 643.2307 .

8ah: This product was synthesized using procedure $\mathbf{B}$ and isolated in (70\% yield, $36.6 \mathrm{mg}$ ) as orange yellow solid after stirring the reaction mixture for $1.5 \mathrm{~h} .{ }^{1} \mathrm{H}$ NMR $\left(\mathrm{CDCl}_{3}, 500 \mathrm{MHz}\right): \delta 7.46-7.33(\mathrm{~m}, 4 \mathrm{H}), 7.17(\mathrm{br}$,<smiles>COC(=O)C1(C(C)(C)C)Cc2cc(C(=Nc3ccc(N(C)C)cc3)c3ccccc3)c(C(C)(C)C)c(C(C)(C)C)c2C1</smiles>
$1 \mathrm{H}), 7.05(\mathrm{~s}, 1 \mathrm{H}), 6.57(\mathrm{~d}, 2 \mathrm{H}, J=7.9 \mathrm{~Hz}), 6.50(\mathrm{~d}, 2 \mathrm{H}, J=8.0 \mathrm{~Hz}), 3.77$ $(\mathrm{s}, 6 \mathrm{H}), 3.70(\mathrm{~s}, 2 \mathrm{H}), 3.60(\mathrm{~s}, 2 \mathrm{H}), 2.83(\mathrm{~s}, 6 \mathrm{H}), 0.42(\mathrm{~s}, 9 \mathrm{H}), 0.26(\mathrm{~s}$, $9 \mathrm{H}) ;{ }^{13} \mathrm{C}$ NMR $\left(\mathrm{CDCl}_{3}, 125 \mathrm{MHz}\right): 172.0,159.2,153.2,147.4,144.6$, $140.5,139.3,138.7,135.6,129.0,126.0,124.8,124.6,123.8,122.0$, 
113.1, 104.2, 104.0, 59.0, 53.1, 41.1, 41.0, 0.6, -0.3; HRMS (ESI) calcd for $\mathrm{C}_{36} \mathrm{H}_{45} \mathrm{~N}_{2} \mathrm{O}_{5} \mathrm{Si}_{2}[\mathrm{M}+\mathrm{H}]^{+} 641.2867$ found 641.2869 .

8ai: This product was synthesized using procedure $\mathbf{B}$ and isolated in (58\% yield, $22.7 \mathrm{mg}$ ) as white solid after stirring the reaction mixture for $1.5 \mathrm{~h} .{ }^{1} \mathrm{H}$ NMR $\left(\mathrm{CDCl}_{3}, 500 \mathrm{MHz}\right): \delta 8.38(\mathrm{~s}, 1 \mathrm{H}), 7.99-7.88(\mathrm{~m}, 1 \mathrm{H})$, 7.63-7.51 (m, 2H), 7.50-7.38 (m, 6H), $7.34(\mathrm{~s}, 1 \mathrm{H}), 7.07(\mathrm{~s}, 1 \mathrm{H}), 3.73(\mathrm{~s}, 6 \mathrm{H}), 3.64(\mathrm{~s}, 2 \mathrm{H}), 3.55(\mathrm{~s}, 2 \mathrm{H}), 0.48$

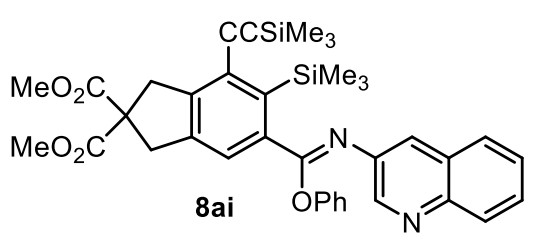

(s, 9H), $0.23(\mathrm{~s}, 9 \mathrm{H}) ;{ }^{13} \mathrm{C} \mathrm{NMR}\left(\mathrm{CDCl}_{3}, 125 \mathrm{MHz}\right): \delta$ 171.8, 163.4, 152.8, 147.2 , 145.4, 144.9, 140.8, 139.8, 139.2, 137.0, 129.4, 129.0, 128.2, $127.9,127.3,126.7,126.5,125.8,125.4,124.6,121.9,104.7,103.7$, 59.1, 53.1, 41.0, 40.9, 0.8, -0.4; HRMS (ESI) calcd for $\mathrm{C}_{37} \mathrm{H}_{41} \mathrm{~N}_{2} \mathrm{O}_{5} \mathrm{Si}_{2}$ $[\mathrm{M}+\mathrm{H}]^{+} 649.2554$ found 649.2586 .

8ca: This product was synthesized using procedure $\mathbf{B}$ and isolated in (73\% yield, $34.6 \mathrm{mg}$ ) as yellow solid after stirring the reaction mixture for $5 \mathrm{~h} .{ }^{1} \mathrm{H}$ NMR $\left(\mathrm{CDCl}_{3}, 500 \mathrm{MHz}\right): \delta 7.75(\mathrm{~d}, 2 \mathrm{H}, J=7.4 \mathrm{~Hz}), 7.52-7.42$<smiles>CC(C)(C)c1c(/C(=N/c2ccccc2)c2ccccc2)cc2c(c1C(C)(C)C)CC1(C2)c2ccccc2-c2ccccc21</smiles>
$(\mathrm{m}, 3 \mathrm{H}), 7.39(\mathrm{~d}, 3 \mathrm{H}, J=8.6 \mathrm{~Hz}), 7.29-7.20(\mathrm{~m}, 5 \mathrm{H}), 7.19-7.10(\mathrm{~m}, 3 \mathrm{H}), 7.01-$ $7.93(\mathrm{~m}, 1 \mathrm{H}), 6.74(\mathrm{~d}, 2 \mathrm{H}, \mathrm{J}=7.2 \mathrm{~Hz}), 3.49(\mathrm{~s}, 2 \mathrm{H}), 3.40(\mathrm{~s}, 2 \mathrm{H}), 0.52(\mathrm{~s}, 9 \mathrm{H}), 0.15$ (s, 9H); ${ }^{13} \mathrm{C} \mathrm{NMR}\left(\mathrm{CDCl}_{3}, 125 \mathrm{MHz}\right): \delta 161.6,153.1,152.0,147.8,146.6,143.2$, $139.6,138.7,137.3,129.3,128.4,127.7,127.5,126.2,125.4,125.1,123.3$, $122.5,122.4,122.0,119.9,104.3,103.8,56.5,45.7,45.4,0.9,-0.4$; HRMS (ESI) calcd for $\mathrm{C}_{42} \mathrm{H}_{42} \mathrm{NOSi}_{2}[\mathrm{M}+\mathrm{H}]^{+} 632.2805$ found 632.2789 .

8ha: This product was synthesized using procedure B and isolated in ( $82 \%$ yield, $29.4 \mathrm{mg}$ ) as yellow solid<smiles>Cc1c(C(=Nc2ccccc2)Oc2ccccc2)cc2c(c1C(C)(C)C)C(=O)c1ccccc1-2</smiles>
after stirring the reaction mixture for $2 \mathrm{~h}$. NMR at higher temperature $(333 \mathrm{~K}) ;{ }^{1} \mathbf{H}$ NMR $\left(\mathrm{CDCl}_{3}, 500 \mathrm{MHz}\right): \delta 7.60(\mathrm{~d}, 2 \mathrm{H}, J=6.4 \mathrm{~Hz}), 7.42(\mathrm{t}, 1 \mathrm{H}, J=7.2 \mathrm{~Hz}), 7.39-$ $7.24(\mathrm{~m}, 4 \mathrm{H}), 7.22-7.01(\mathrm{~m}, 9 \mathrm{H}), 6.96(\mathrm{t}, 1 \mathrm{H}, \mathrm{J}=7.0 \mathrm{~Hz}), 6.90(\mathrm{br}, 2 \mathrm{H}), 0.47(\mathrm{~s}, 9 \mathrm{H})$, 0.42 (s, 9H); $\left.{ }^{13} \mathrm{C} \mathrm{NMR} \mathrm{(CDCl}, 125 \mathrm{MHz}\right): \delta$ 195.5, 154.6, 153.8 (br), 145.9 (br), 143.3, 143.2, 141.0, 134.5, 134.0, 129.3, 129.2, 128.5, 124.0, 122.5, 121.3, 120.1, 4.1, 3.0; HRMS (ESI) calcd for $\mathrm{C}_{32} \mathrm{H}_{34} \mathrm{NO}_{2} \mathrm{Si}_{2}[\mathrm{M}+\mathrm{H}]^{+} 520.2128$ found 520.2136 .

8fa: This product was synthesized using procedure $\mathbf{B}$ and isolated in (68\% yield, $35.7 \mathrm{mg}$ ) as yellow solid after stirring the reaction mixture for $2 \mathrm{~h} .{ }^{1} \mathrm{H}$ NMR $\left(\mathrm{CDCl}_{3}, 500 \mathrm{MHz}\right): \delta 7.51-7.36(\mathrm{~m}, 2 \mathrm{H}), 7.35-7.26(\mathrm{~m}$, $2 \mathrm{H}), 7.24-7.17(\mathrm{~m}, 1 \mathrm{H}), 7.13-6.99(\mathrm{~m}, 2 \mathrm{H}), 6.93(\mathrm{~s}, 1 \mathrm{H}), 6.90-6.80(\mathrm{~m}, 1 \mathrm{H}), 6.70-6.57(\mathrm{~m}, 2 \mathrm{H}), 3.74(\mathrm{~s}, 6 \mathrm{H})$,

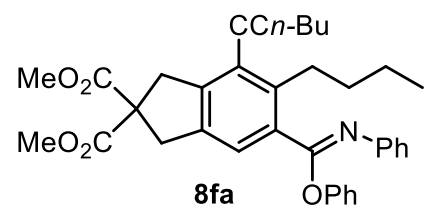
$3.62(\mathrm{~s}, 2 \mathrm{H}), 3.50(\mathrm{~s}, 2 \mathrm{H}), 2.83(\mathrm{br}, 2 \mathrm{H}), 2.47(\mathrm{t}, 2 \mathrm{H}, \mathrm{J}=6.4 \mathrm{~Hz}), 1.76-1.40(\mathrm{~m}$, $8 \mathrm{H}), 0.96(\mathrm{t}, 6 \mathrm{H}, \mathrm{J}=7.0 \mathrm{~Hz}) ;{ }^{13} \mathrm{C} \mathrm{NMR}\left(\mathrm{CDCl}_{3}, 125 \mathrm{MHz}\right): \delta 171.9,160.8,153.0$, $146.7,144.4,141.4,136.8,130.8,129.4,128.5,125.1,123.6,123.2,122.0$, 121.7, 120.9, 98.7, 59.6, 53.0, 41.0, 40.6, 32.6, 32.4, 30.9, 23.5, 22.0, 19.4, 14.0, 13.6; HRMS (ESI) calcd for $\mathrm{C}_{36} \mathrm{H}_{40} \mathrm{NO}_{5}[\mathrm{M}+\mathrm{H}]^{+} 566.2906$ found 566.2926 .

8ga: This product was synthesized using procedure $\mathbf{B}$ and isolated in (53\% yield, $24.2 \mathrm{mg}$ ) as orange solid after stirring the reaction mixture for $30 \mathrm{~min} .{ }^{1} \mathrm{H} ~ \mathrm{NMR}\left(\mathrm{CDCl}_{3}, 500 \mathrm{MHz}\right): \delta 7.75(\mathrm{~d}, 2 \mathrm{H}, J=7.2 \mathrm{~Hz}), 7.50-$<smiles>CCCCOC(=O)c1c2c(cc(/C(=N\c3ccccc3)Oc3ccccc3)c1CCCC)CN([AsH3])C2</smiles>
$7.35(\mathrm{~m}, 2 \mathrm{H}), 7.31(\mathrm{~d}, 2 \mathrm{H}, \mathrm{J}=7.6 \mathrm{~Hz}), 7.29-7.16(\mathrm{~m}, 3 \mathrm{H}), 7.13-6.97(\mathrm{~m}, 2 \mathrm{H}), 6.96-$ $6.78(\mathrm{~m}, 2 \mathrm{H}), 6.68-6.51(\mathrm{~m}, 2 \mathrm{H}), 4.58(\mathrm{~s}, 2 \mathrm{H}), 4.51(\mathrm{~s}, 2 \mathrm{H}), 2.91-2.75(\mathrm{~m}, 2 \mathrm{H}), 2.46$ $(\mathrm{d}, 2 \mathrm{H}, J=6.8 \mathrm{~Hz}), 2.41(\mathrm{~s}, 3 \mathrm{H}), 1.75-1.34(\mathrm{~m}, 8 \mathrm{H}), 0.97(\mathrm{t}, 3 \mathrm{H}, J=7.3 \mathrm{~Hz}), 0.95(\mathrm{t}$, $3 \mathrm{H}, J=7.4 \mathrm{~Hz}) ;{ }^{13} \mathrm{C}$ NMR $\left(\mathrm{CDCl}_{3}, 125 \mathrm{MHz}\right): \delta 160.0,152.8,146.5,143.7,142.3$, 
140.6, 133.8, 133.2, 131.7, 129.9, 129.4, 128.6, 127.5, 125.3, 123.3, 121.9, 121.5, 119.9, 99.9, 75.8, 54.2, 54.0, 32.5, 32.3, 30.7, 23.4, 22.0, 21.5, 19.3, 13.9, 13.6; HRMS (ESI) calcd for $\mathrm{C}_{38} \mathrm{H}_{41} \mathrm{~N}_{2} \mathrm{O}_{3} \mathrm{~S}[\mathrm{M}+\mathrm{H}]^{+} 605.2838$ found 605.2823 .

9aa: This product was synthesized using procedure $\mathbf{C}$ and isolated in (58\% yield, $29.4 \mathrm{mg}$ ) as red yellow solid in an inseparable mixture of two tautomers (1:1) after stirring the reaction mixture for $1.5 \mathrm{~h} .{ }^{1} \mathbf{H ~ N M R}$ $\left(\mathrm{CDCl}_{3}, 500 \mathrm{MHz}\right): \delta 10.26(\mathrm{br}, 1 \mathrm{H}), 7.88(\mathrm{~d}, 2 \mathrm{H}, J=7.0 \mathrm{~Hz}), 7.67(\mathrm{~d}, 2 \mathrm{H}, J=6.8 \mathrm{~Hz}), 7.56(\mathrm{~d}, 2 \mathrm{H}, J=7.8 \mathrm{~Hz})$, $7.33(\mathrm{t}, 2 \mathrm{H}, J=7.0 \mathrm{~Hz}), 7.29(\mathrm{~d}, 2 \mathrm{H}, J=7.3 \mathrm{~Hz}), 7.23(\mathrm{t}, 2 \mathrm{H}, J=7.4 \mathrm{~Hz}), 7.20-7.11(\mathrm{~m}, 3 \mathrm{H}), 7.07(\mathrm{t}, 1 \mathrm{H}, J=6.6$ $\mathrm{Hz}), 7.01(\mathrm{~s}, 1 \mathrm{H}), 6.93(\mathrm{~s}, 1 \mathrm{H}), 6.80(\mathrm{~s}, 1 \mathrm{H}), 6.66(\mathrm{~d}, 2 \mathrm{H}, J=7.7 \mathrm{~Hz}), 3.83(\mathrm{~s}, 3 \mathrm{H}), 3.79-3.61(\mathrm{~m}, 13 \mathrm{H}), 3.58$

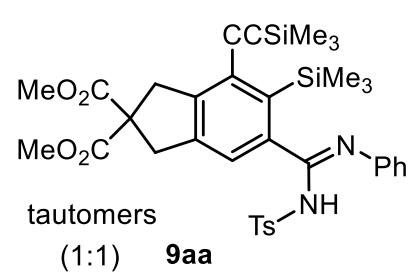
$(\mathrm{s}, 2 \mathrm{H}), 3.52(\mathrm{~s}, 2 \mathrm{H}), 2.41(\mathrm{~s}, 6 \mathrm{H}), 0.42(\mathrm{~s}, 9 \mathrm{H}), 0.27(\mathrm{~s}, 9 \mathrm{H}), 0.26(\mathrm{~s}, 9 \mathrm{H}), 0.22(\mathrm{~s}$, $9 \mathrm{H}) ;{ }^{13} \mathrm{C}$ NMR $\left(\mathrm{CDCl}_{3}, 125 \mathrm{MHz}\right): \delta 172.4,171.9,171.5,163.8,145.7,145.6$, $143.3,142.4,140.6,140.2,140.1,139.6,139.2,138.8,137.3,136.8,129.4$, $129.2,129.1,126.9,126.3,126.2,125.9,125.5,124.1,123.8,122.8,120.8$, $104.8,104.5,103.7,103.6,59.1,59.0,53.1,41.1,41.0,40.9,0.6,0.5,-0.3,-$ 0.4; HRMS (ESI) calcd for $\mathrm{C}_{35} \mathrm{H}_{43} \mathrm{~N}_{2} \mathrm{O}_{6} \mathrm{Si}_{2} \mathrm{~S}[\mathrm{M}+\mathrm{H}]^{+} 675.2380$ found 675.2360 .

9ab: This product was synthesized using procedure $\mathbf{C}$ and isolated in (70\% yield, $26.7 \mathrm{mg}$ ) as light yellow solid as an inseparable mixture of two tautomers (1:1) after stirring the reaction mixture for $1.5 \mathrm{~h} .{ }^{1} \mathbf{H ~ N M R}$ $\left(\mathrm{CDCl}_{3}, 500 \mathrm{MHz}\right): \delta 9.92(\mathrm{br}, 1 \mathrm{H}), 7.59(\mathrm{~d}, 2 \mathrm{H}, J=7.6 \mathrm{~Hz}), 7.38(\mathrm{t}, 2 \mathrm{H}, J=7.3 \mathrm{~Hz}), 7.29(\mathrm{~s}, 1 \mathrm{H}), 7.21(\mathrm{~d}, 1 \mathrm{H}$, $J=7.2 \mathrm{~Hz}), 7.15(\mathrm{~d}, 2 \mathrm{H}, J=7.3 \mathrm{~Hz}), 7.07(\mathrm{~d}, 1 \mathrm{H}, J=7.2 \mathrm{~Hz}), 7.03(\mathrm{~s}, 1 \mathrm{H}), 6.80(\mathrm{~s}, 1 \mathrm{H}), 6.67(\mathrm{~d}, 2 \mathrm{H}, J=7.8 \mathrm{~Hz})$,

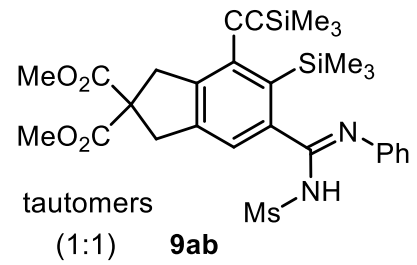
3.85-3.59 (m, 18H), $3.58(\mathrm{~s}, 2 \mathrm{H}), 3.11(\mathrm{~s}, 3 \mathrm{H}), 3.04(\mathrm{~s}, 3 \mathrm{H}), 0.42(\mathrm{~s}, 9 \mathrm{H}), 0.36$ (s, 9H), $0.26(\mathrm{~s}, 9 \mathrm{H}), 0.23(\mathrm{~s}, 9 \mathrm{H}) ;{ }^{13} \mathrm{C} \mathrm{NMR}\left(\mathrm{CDCl}_{3}, 125 \mathrm{MHz}\right): \delta 172.5,171.9$, $171.7,171.4,164.5,164.0,145.9,145.7,140.8,140.3,139.7,139.2,138.8$, $138.3,137.4,136.7,129.2,126.5,126.3,125.9,125.6,124.1,122.8,120.9$, $104.8,104.7,103.7,103.5,59.0,53.2,43.4,42.9,41.1,40.9,0.7,0.5,-0.3,-$ 0.4; HRMS (ESI) calcd for $\mathrm{C}_{29} \mathrm{H}_{39} \mathrm{~N}_{2} \mathrm{O}_{6} \mathrm{Si}_{2} \mathrm{~S}[\mathrm{M}+\mathrm{H}]^{+} 599.2067$ found 599.2076.

9ac: This product was synthesized using procedure $\mathbf{C}$ and isolated in (79\% yield, $35.5 \mathrm{mg}$ ) as light yellow oil after stirring the reaction mixture for $1.5 \mathrm{~h}$. NMR at higher temperature $(333 \mathrm{~K}) ;{ }^{1} \mathbf{H}$ NMR $\left(\mathrm{CDCl}_{3}, 500\right.$ MHz): $\delta 7.55(\mathrm{br}, 2 \mathrm{H}), 7.24(\mathrm{~d}, 2 \mathrm{H}, J=7.8 \mathrm{~Hz}), 6.98(\mathrm{t}, 2 \mathrm{H}, J=7.2 \mathrm{~Hz}), 6.80(\mathrm{t}, 1 \mathrm{H}, J=6.8 \mathrm{~Hz}), 6.49(\mathrm{br}, 1 \mathrm{H})$, $6.39(\mathrm{~d}, 2 \mathrm{H}, J=6.7 \mathrm{~Hz}), 3.99(\mathrm{br}, 1 \mathrm{H}), 3.88-3.67(\mathrm{~m}, 7 \mathrm{H}), 3.63(\mathrm{~s}, 2 \mathrm{H}), 3.50-3.29(\mathrm{~m}, 2 \mathrm{H}), 2.44(\mathrm{~s}, 3 \mathrm{H}), 1.91$

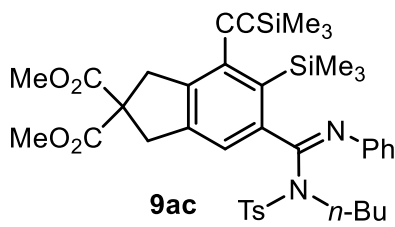

(br, 1H), $1.72(\mathrm{br}, 1 \mathrm{H}), 1.40-1.18(\mathrm{br}, 2 \mathrm{H}), 0.92-0.81(\mathrm{br}, 3 \mathrm{H}), 0.27$ (s, 9H), 0.22 $(\mathrm{s}, 9 \mathrm{H}) ;{ }^{13} \mathrm{C}$ NMR $\left(\mathrm{CDCl}_{3}, 125 \mathrm{MHz}\right): \delta 171.8,171.7,155.2,147.0,144.4,143.5$, 140.9 (br), 138.8, 138.5, 136.9, 128.9, 128.3, 128.1, 125.7, 125.2, 122.8, 122.0, 104.3, 104.2, 59.1, 52.8, 48.4, 41.1, 41.0, 31.8, 29.6, 21.4, 20.1, 13.4, 0.5, -0.5; HRMS (ESI) calcd for $\mathrm{C}_{39} \mathrm{H}_{51} \mathrm{~N}_{2} \mathrm{O}_{6} \mathrm{Si}_{2} \mathrm{~S}[\mathrm{M}+\mathrm{H}]^{+} 731.3006$ found 731.3016 .

9ad: This product was synthesized using procedure $\mathbf{C}$ and isolated in (68\% yield, $29.3 \mathrm{mg}$ ) as orange oil after stirring the reaction mixture for $1.5 \mathrm{~h}$. NMR at higher temperature $(333 \mathrm{~K}) ;{ }^{1} \mathrm{H} \mathrm{NMR}\left(\mathrm{CDCl}_{3}, 500 \mathrm{MHz}\right)$ : $\delta 7.56(\mathrm{br}, 2 \mathrm{H}), 7.23(\mathrm{~d}, 2 \mathrm{H}, J=7.6 \mathrm{~Hz}), 6.98(\mathrm{t}, 1 \mathrm{H}, J=6.9 \mathrm{~Hz}), 6.80(\mathrm{t}, 1 \mathrm{H}, J=6.7 \mathrm{~Hz}), 6.48(\mathrm{br}, 1 \mathrm{H}), 6.39(\mathrm{~d}$,

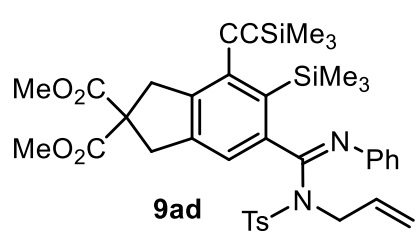

$2 \mathrm{H}, J=6.7 \mathrm{~Hz}), 6.10-5.95(\mathrm{~m}, 1 \mathrm{H}), 5.24-5.04(\mathrm{~m}, 2 \mathrm{H}), 4.73(\mathrm{br}, 1 \mathrm{H}), 4.46(\mathrm{br}$, $1 \mathrm{H}), 3.80(\mathrm{~s}, 3 \mathrm{H}), 3.75(\mathrm{~s}, 3 \mathrm{H}), 3.63(\mathrm{~s}, 2 \mathrm{H}), 3.47-3.28(\mathrm{~m}, 2 \mathrm{H}), 2.44(\mathrm{~s}, 3 \mathrm{H}), 0.27$ (s, 9H), 0.23 (s, 9H); ${ }^{13} \mathrm{C}$ NMR $\left(\mathrm{CDCl}_{3}, 125 \mathrm{MHz}\right): \delta 171.8,171.7,155.0,146.9$, $144.5,143.6,140.9,138.9,138.3,136.6,134.0,128.9,128.5,128.1,125.7$, 
125.4, 123.0, 118.4, 104.4, 104.1, 59.0, 52.8, 50.2, 41.1, 40.9, 21.4, 0.5, -0.5; HRMS (ESI) calcd for $\mathrm{C}_{38} \mathrm{H}_{47} \mathrm{~N}_{2} \mathrm{O}_{6} \mathrm{Si}_{2} \mathrm{~S}[\mathrm{M}+\mathrm{H}]^{+} 715.2693$ found 715.2724 .

9ae: This product was synthesized using procedure $\mathbf{C}$ and isolated in ( $89 \%$ yield, $42.1 \mathrm{mg}$ ) as yellow solid after stirring the reaction mixture for $1.5 \mathrm{~h}$. NMR at higher temperature $(333 \mathrm{~K}) ;{ }^{1} \mathbf{H ~ N M R}\left(\mathrm{CDCl}_{3}, 500 \mathrm{MHz}\right)$ : 6 7.5-7.19 (br, 7H), $7.16(\mathrm{~d}, 2 \mathrm{H}, J=6.9 \mathrm{~Hz}), 6.97(\mathrm{t}, 2 \mathrm{H}, J=7.0 \mathrm{~Hz}), 6.80(\mathrm{t}, 1 \mathrm{H}, J=7.5 \mathrm{~Hz}), 6.37(\mathrm{~d}, 2 \mathrm{H}, J=$ $7.0 \mathrm{~Hz}), 5.64(\mathrm{br}, 1 \mathrm{H}), 4.85(\mathrm{br}, 1 \mathrm{H}), 3.81(\mathrm{~s}, 3 \mathrm{H}), 3.73(\mathrm{~s}, 3 \mathrm{H}), 3.59(\mathrm{~d}, 2 \mathrm{H}, J=10.9 \mathrm{~Hz}), 3.34(\mathrm{~d}, 1 \mathrm{H}, J=17.5$<smiles>CC(=O)C1(C(C)=O)Cc2cc(/C(=N/c3ccccc3)N(C)Cc3ccccc3)c(C(C)(C)C)c(C(C)(C)C)c2C1</smiles>
$\mathrm{Hz}), 3.11(\mathrm{~d}, 1 \mathrm{H}, J=17.4 \mathrm{~Hz}), 2.42(\mathrm{~s}, 3 \mathrm{H}), 0.25(\mathrm{~s}, 9 \mathrm{H}), 0.23(\mathrm{~s}, 9 \mathrm{H}) ;{ }^{13} \mathrm{C}$ NMR $\left(\mathrm{CDCl}_{3}, 125 \mathrm{MHz}\right): \delta 171.8,171.7,155.2,146.7,144.4,143.6,138.6,138.2$, $137.8,136.4,128.9,128.8,128.6,128.1,127.2,125.6,125.5,123.0,121.9$, 104.3, 104.2, 77.7, 77.1, 76.9, 76.6, 58.8, 52.8, 50.7, 41.1, 40.8, 29.6, 21.3, 0.5, -0.5 ; HRMS (ESI) calcd for $\mathrm{C}_{42} \mathrm{H}_{49} \mathrm{~N}_{2} \mathrm{O}_{6} \mathrm{Si}_{2} \mathrm{~S}[\mathrm{M}+\mathrm{H}]^{+} 765.2850$ found 765.2872 .

9af: This product was synthesized using procedure $\mathbf{C}$ and isolated in ( $81 \%$ yield, $44.7 \mathrm{mg}$ ) as light yellow solid after stirring the reaction mixture for $1.5 \mathrm{~h} .{ }^{1} \mathrm{H} \mathrm{NMR}\left(\mathrm{CDCl}_{3}, 500 \mathrm{MHz}\right): \delta 7.71(\mathrm{~d}, 2 \mathrm{H}, J=7.6 \mathrm{~Hz}), 7.31$ $(\mathrm{d}, 2 \mathrm{H}, J=8.0 \mathrm{~Hz}), 7.30-7.20(\mathrm{~m}, 5 \mathrm{H}), 6.99(\mathrm{t}, 2 \mathrm{H}, J=7.6 \mathrm{~Hz}), 6.82(\mathrm{t}, 1 \mathrm{H}, J=7.3 \mathrm{~Hz}), 6.78(\mathrm{~s}, 1 \mathrm{H}), 6.41(\mathrm{~d}$,

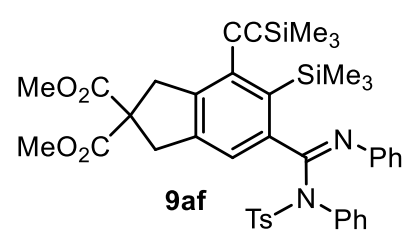
$2 \mathrm{H}, J=7.8 \mathrm{~Hz}), 3.77(\mathrm{~s}, 3 \mathrm{H}), 3.75(\mathrm{~s}, 3 \mathrm{H}), 3.61-3.48(\mathrm{~m}, 2 \mathrm{H}), 3.49(\mathrm{~s}, 2 \mathrm{H}), 2.48$ (S, 3H), $0.20(\mathrm{~s}, 9 \mathrm{H}), 0.18(\mathrm{~s}, 9 \mathrm{H}) ;{ }^{13} \mathrm{C} \mathrm{NMR}\left(\mathrm{CDCl}_{3}, 125 \mathrm{MHz}\right): \delta 171.8,155.3$, $146.5,144.4,144.0,140.1,139.2,137.7,137.3,136.6,131.5,129.6,128.8$, $128.7,128.6,128.2,125.7,125.6,123.2,122.4,104.4,104.0,59.0,53.1,41.0$, 40.8, 21.7, 0.6, -0.4; HRMS (ESI) calcd for $\mathrm{C}_{41} \mathrm{H}_{47} \mathrm{~N}_{2} \mathrm{O}_{6} \mathrm{Si}_{2} \mathrm{~S}[\mathrm{M}+\mathrm{H}]^{+} 751.2693$ found 751.2707 .

9ag: This product was synthesized using procedure $\mathbf{C}$ and isolated in ( $36 \%$ yield, $19.2 \mathrm{mg}$ ) as orange yellow solid after stirring the reaction mixture for $1.5 \mathrm{~h} .{ }^{1} \mathrm{H} \mathrm{NMR}\left(\mathrm{CDCl}_{3}, 500 \mathrm{MHz}\right): \delta 7.40(\mathrm{t}, 2 \mathrm{H}, J=7.5 \mathrm{~Hz}), 7.35$<smiles>CC(=O)OC1(C(C)=O)Cc2cc(/C(=N\c3ccccc3)N(C)C(C)=O)c(C(C)(C)C)c(C(C)(C)C)c2C1</smiles>
$(\mathrm{t}, 1 \mathrm{H}, J=7.1 \mathrm{~Hz}), 7.24-7.15(\mathrm{~m}, 4 \mathrm{H}), 7.04(\mathrm{~d}, 2 \mathrm{H}, \mathrm{J}=7.7 \mathrm{~Hz}), 6.79(\mathrm{~s}, 1 \mathrm{H}), 3.81-$ $3.71(\mathrm{~m}, 8 \mathrm{H}), 3.59(\mathrm{~s}, 2 \mathrm{H}), 2.33(\mathrm{~s}, 3 \mathrm{H}), 1.14(\mathrm{~s}, 9 \mathrm{H}), 0.52(\mathrm{~s}, 9 \mathrm{H}), 0.27(\mathrm{~s}, 9 \mathrm{H})$; ${ }^{13} \mathrm{C} \mathrm{NMR}\left(\mathrm{CDCl}_{3}, 125 \mathrm{MHz}\right): \delta 168.4,151.3,144.7,142.3,140.7,139.6,139.4$, 138.5, 129.1, 128.8, 128.0, 127.8, 126.4, 125.5, 121.2, 104.1, 84.7, 59.2, 53.1, 41.1, 41.0, 27.4, 21.4, 0.9, -0.3; HRMS (ESI) calcd for $\mathrm{C}_{40} \mathrm{H}_{51} \mathrm{~N}_{2} \mathrm{O}_{8} \mathrm{Si}_{2} \mathrm{~S}[\mathrm{M}+\mathrm{H}]^{+}$ 775.2905 found 775.2914 .

9ah: This product was synthesized using procedure $\mathbf{C}$ and isolated in (71\% yield, $32.6 \mathrm{mg}$ ) as yellow solid after stirring the reaction mixture for $1.5 \mathrm{~h} .{ }^{1} \mathrm{H} \mathrm{NMR}\left(\mathrm{CDCl}_{3}, 500 \mathrm{MHz}\right): \delta 8.24(\mathrm{~s}, 1 \mathrm{H}), 7.55(\mathrm{~d}, 2 \mathrm{H}, J=7.8$ 9ah $\mathrm{Hz}), 7.43(\mathrm{~d}, 2 \mathrm{H}, J=7.4 \mathrm{~Hz}), 7.38-7.22(\mathrm{~m}, 10 \mathrm{H}), 6.65(\mathrm{~s}, 1 \mathrm{H}), 3.81(\mathrm{~s}, 3 \mathrm{H}), 3.76$ $(\mathrm{s}, 3 \mathrm{H}), 3.69-3.54(\mathrm{~m} .2 \mathrm{H}), 3.46(\mathrm{~s}, 2 \mathrm{H}), 2.46(\mathrm{~s}, 3 \mathrm{H}), 0.27(\mathrm{~s}, 9 \mathrm{H}), 0.25(\mathrm{~s}, 9 \mathrm{H})$; ${ }^{13} \mathrm{C} \mathrm{NMR}\left(\mathrm{CDCl}_{3}, 125 \mathrm{MHz}\right): \delta$ 172.1, 172.0, 162.3, 159.5, 144.1, 142.2, 139.0, $137.9,137.3,137.2,134.3,131.3,130.5,129.0,128.8,128.4,124.9,123.7$, 104.5, 103.9, 58.8, 53.1, 41.2, 41.0, 21.6, 0.7, -0.3; HRMS (ESI) calcd for $\mathrm{C}_{42} \mathrm{H}_{48} \mathrm{~N}_{3} \mathrm{O}_{6} \mathrm{Si}_{2} \mathrm{~S}[\mathrm{M}+\mathrm{H}]^{+} 778.2802$ found 778.2809. 
10aa: This product was synthesized using procedure $\mathbf{D}$ and isolated in ( $88 \%$ yield, $37.8 \mathrm{mg}$ ) as light yellow<smiles>CC(=O)C1(C(C)(C)C)Cc2cc(C(=O)Nc3ccccc3)c(C(C)(C)C)c(C(C)(C)C)c2C1</smiles>
solid after stirring the reaction mixture for $1.5 \mathrm{~h} .{ }^{1} \mathrm{H} \mathrm{NMR}\left(\mathrm{CDCl}_{3}, 500 \mathrm{MHz}\right)$ : $\delta 7.58(\mathrm{~d}, 2 \mathrm{H}, J=7.6 \mathrm{~Hz}), 7.41-7.32(\mathrm{~m}, 3 \mathrm{H}), 7.23(\mathrm{~s}, 1 \mathrm{H}), 7.14(\mathrm{t}, 1 \mathrm{H}, J=7.2$ $\mathrm{Hz}), 3.78(\mathrm{~s}, 6 \mathrm{H}), 3.69(\mathrm{~s}, 2 \mathrm{H}), 3.61(\mathrm{~s}, 2 \mathrm{H}), 0.40(\mathrm{~s}, 9 \mathrm{H}), 0.27(\mathrm{~s}, 9 \mathrm{H}) ;{ }^{13} \mathrm{C} \mathrm{NMR}$ $\left(\mathrm{CDCl}_{3}, 125 \mathrm{MHz}\right): \delta 171.9,169.4,145.2,143.7,140.6,138.6,138.0,129.2$, 126.4, 124.5, 122.3, 119.5, 104.3, 103.8, 59.2, 53.2, 41.0, 40.9, 0.8, -0.3; HRMS (ESI) calcd for $\mathrm{C}_{28} \mathrm{H}_{36} \mathrm{NO}_{5} \mathrm{Si2}[\mathrm{M}+\mathrm{H}]^{+} 522.2132$ found 522.2129 .

10ab: This product was synthesized using procedure $\mathbf{D}$ and isolated in (69\% yield, $23.6 \mathrm{mg}$ ) as white solid

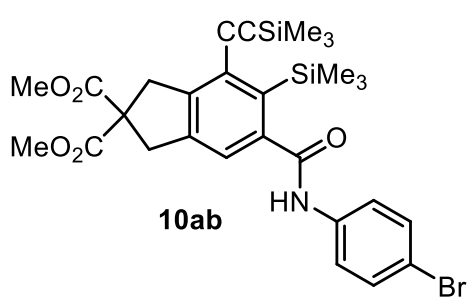
after stirring the reaction mixture for $1.5 \mathrm{~h} .{ }^{1} \mathrm{H}$ NMR $\left(\mathrm{CDCl}_{3}, 500 \mathrm{MHz}\right)$ : ס 7.52-7.44 (m, 4H), $7.37(\mathrm{~s}, 1 \mathrm{H}), 7.21(\mathrm{~s}, 1 \mathrm{H}), 3.78(\mathrm{~s}, 6 \mathrm{H}), 3.69(\mathrm{~s}, 2 \mathrm{H})$, $3.61(\mathrm{~s}, 2 \mathrm{H}), 0.38(\mathrm{~s}, 9 \mathrm{H}), 0.27(\mathrm{~s}, 9 \mathrm{H}) ;{ }^{13} \mathrm{C} \mathrm{NMR}\left(\mathrm{CDCl}_{3}, 125 \mathrm{MHz}\right): \delta$ $171.8,169.3,145.4,143.3,140.7,138.6,137.0,132.2,126.5,122.2$, 121.0, 117.1, 104.5, 103.7, 59.2, 53.2, 41.0, 40.9, 0.7, -0.3; HRMS (ESI) calcd for $\mathrm{C}_{28} \mathrm{H}_{35} \mathrm{NO}_{5} \mathrm{Si} 2 \mathrm{Br}[\mathrm{M}+\mathrm{H}]^{+} 600.1237$ found 600.1230 .

10ac: This product was synthesized using procedure $\mathbf{D}$ and isolated in (60\% yield, $20.4 \mathrm{mg}$ ) as yellow solid<smiles>CC(=O)c1ccc(NC(=O)c2cc3c(c(C(C)(C)C)c2C(C)(C)C)CC(C(C)=O)(C(C)C)C3)cc1</smiles>
after stirring the reaction mixture for $1.5 \mathrm{~h} .{ }^{1} \mathbf{H}$ NMR $\left(\mathrm{CDCl}_{3}, 500\right.$ $\mathrm{MHz}): \delta 8.04(\mathrm{~d}, 2 \mathrm{H}, J=8.4 \mathrm{~Hz}), 7.67(\mathrm{~d}, 2 \mathrm{H}, J=8.6 \mathrm{~Hz}), 7.52(\mathrm{~s}, 1 \mathrm{H})$, $7.24(\mathrm{~S}, 1 \mathrm{H}), 3.91(\mathrm{~s}, 3 \mathrm{H}), 3.78(\mathrm{~s}, 6 \mathrm{H}), 3.70(\mathrm{~s}, 2 \mathrm{H}), 3.62(\mathrm{~s}, 2 \mathrm{H}), 0.39$

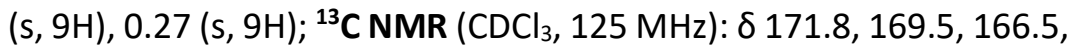
$145.5,143.2,142.0,140.7,138.7,131.1,126.6,126.0,122.2,118.6$, 104.6, 103.7, 59.2, 53.2, 52.1, 41.0, 40.9, 0.7, -0.3; HRMS (ESI) calcd for $\mathrm{C}_{30} \mathrm{H}_{38} \mathrm{NO}_{7} \mathrm{Si}_{2}[\mathrm{M}+\mathrm{H}]^{+} 580.2187$ found 580.2168 .

10ad: This product was synthesized using procedure $\mathbf{D}$ and isolated in ( $56 \%$ yield, $21.6 \mathrm{mg}$ ) as orange<smiles>CC(=O)C1(C(C)=O)Cc2cc(C(=O)Nc3ccc([N+](=O)[O-])cc3)c(C(C)(C)C)c(C(C)(C)C)c2C1</smiles>
yellow solid after stirring the reaction mixture for $1.5 \mathrm{~h} .{ }^{1} \mathrm{H} \mathbf{~ N M R}\left(\mathrm{CDCl}_{3}\right.$, $500 \mathrm{MHz}$ ): $\delta 8.24$ (d, $2 \mathrm{H}, J=8.5 \mathrm{~Hz}), 7.76(\mathrm{~d}, 2 \mathrm{H}, J=8.5 \mathrm{~Hz}), 7.74(\mathrm{~s}, 1 \mathrm{H})$, $7.22(\mathrm{~S}, 1 \mathrm{H}), 3.78(\mathrm{~s}, 6 \mathrm{H}), 3.70(\mathrm{~s}, 2 \mathrm{H}), 3.61(\mathrm{~s}, 2 \mathrm{H}), 0.39(\mathrm{~s}, 9 \mathrm{H}), 0.27(\mathrm{~s}$, $9 \mathrm{H}) ;{ }^{13} \mathrm{C}$ NMR $\left(\mathrm{CDCl}_{3}, 125 \mathrm{MHz}\right): \delta 171.8,169.6,145.8,143.8,143.5$, $142.7,140.8,138.8,126.8,125.3,122.0,118.9,104.9,103.5,59.1,53.2$, 41.0, 40.9, 0.7, -0.3; HRMS (ESI) calcd for $\mathrm{C}_{28} \mathrm{H}_{35} \mathrm{~N}_{2} \mathrm{O}_{7} \mathrm{Si}_{2}[\mathrm{M}+\mathrm{H}]^{+}$ 567.1983 found 567.1973 .

10ae: This product was synthesized using procedure $\mathbf{D}$ and isolated in (71\% yield, $28.5 \mathrm{mg}$ ) as red yellow solid after stirring the reaction mixture for $1.5 \mathrm{~h} .{ }^{1} \mathrm{H} \mathrm{NMR}\left(\mathrm{CDCl}_{3}, 500 \mathrm{MHz}\right): \delta 8.94(\mathrm{~s}, 1 \mathrm{H}), 8.71(\mathrm{~S}, 1 \mathrm{H})$,<smiles>CC(=O)OC1(C(C)=O)Cc2cc(C(=O)Nc3cnc4ccccc4c3)c(C(C)=O)c(C(C)=O)c2C1</smiles>
$8.03(\mathrm{~d}, 1 \mathrm{H}, J=8.4 \mathrm{~Hz}), 7.96(\mathrm{~s}, 1 \mathrm{H}), 7.84(\mathrm{~d}, 1 \mathrm{H}, J=8.2 \mathrm{~Hz}), 7.64(\mathrm{t}, 1 \mathrm{H}, J$ $=7.6 \mathrm{~Hz}), 7.55(\mathrm{~d}, 1 \mathrm{H}, J=7.6 \mathrm{~Hz}), 3.77(\mathrm{~s}, 6 \mathrm{H}), 3.70(\mathrm{~s}, 2 \mathrm{H}), 3.62(\mathrm{~s}, 2 \mathrm{H})$, $0.40(\mathrm{~s}, 9 \mathrm{H}), 0.27(\mathrm{~s}, 9 \mathrm{H}) ;{ }^{13} \mathrm{C}$ NMR $\left(\mathrm{CDCl}_{3}, 125 \mathrm{MHz}\right): \delta 171.8,170.0$, $145.5,145.4,143.5,143.0,140.7,138.8,131.6,129.0,128.5,128.3$, 128.0, 127.4, 126.6, 123.7, 122.2, 104.6, 103.7, 59.2, 53.2, 41.0, 40.9, 0.8, -0.3 ; HRMS (ESI) calcd for $\mathrm{C}_{31} \mathrm{H}_{37} \mathrm{~N}_{2} \mathrm{O}_{5} \mathrm{Si}_{2}[\mathrm{M}+\mathrm{H}]^{+} 573.2241$ found 573.2222 . 
7aa': These products were isolated as an inseparable mixture using procedure $\mathbf{F}$ and isolated in $82 \%$ yield (23.6 mg) as light yellow oil after stirring the reaction mixture for $1.5 \mathrm{~h} .{ }^{1} \mathbf{H} \mathbf{~ N M R}\left(\mathrm{CDCl}_{3}, 500 \mathrm{MHz}\right): \delta 7.45$ $(\mathrm{t}, 2 \mathrm{H}, J=7.8 \mathrm{~Hz}), 7.38(\mathrm{t}, 1 \mathrm{H}, J=7.2 \mathrm{~Hz}), 7.22(\mathrm{~d}, 2 \mathrm{H}, J=7.3 \mathrm{~Hz}), 6.99(\mathrm{~s}, 1 \mathrm{H}), 3.76(\mathrm{~s}, 6 \mathrm{H}), 3.67(\mathrm{~s}, 2 \mathrm{H}), 3.59$

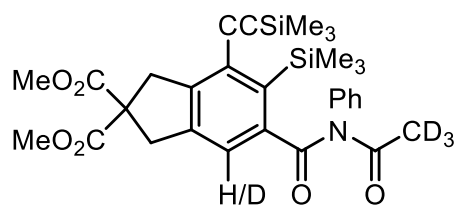

7aa' $\quad(1.5 / 1)$

$(\mathrm{s}, 2 \mathrm{H}), 2.15(\mathrm{~s}, 3 \mathrm{H}), 0.42(\mathrm{~s}, 9 \mathrm{H}), 0.25(\mathrm{~s}, 9 \mathrm{H}) ;{ }^{13} \mathrm{C} \mathrm{NMR}\left(\mathrm{CDCl}_{3}, 125 \mathrm{MHz}\right): \delta$ $173.2,172.5,171.9,144.6,143.5,139.7,139.6,139.4,138.8,129.8,128.9$, $128.8,126.4,120.4,104.0,103.9,59.2,53.1,41.0,40.9,25.7,25.5,25.4$, 25.2, 0.9, -0.3 HRMS (ESI) calcd for $\mathrm{C}_{30} \mathrm{H}_{35} \mathrm{D}_{3} \mathrm{NO}_{6} \mathrm{Si}_{2}[\mathrm{M}+\mathrm{H}]^{+}\left(7 \mathrm{aa}^{\prime}-\mathrm{H}\right)$ 567.2426 found 567.2426 , and $\mathrm{C}_{30} \mathrm{H}_{34} \mathrm{D}_{4} \mathrm{NO}_{6} \mathrm{Si}_{2}[\mathrm{M}+\mathrm{H}]^{+}$(7aa'-D) 568.2489 found 568.2482 . 


\section{${ }^{1} \mathrm{H}$ and ${ }^{13} \mathrm{C}$ NMR Spectra of Substrates}
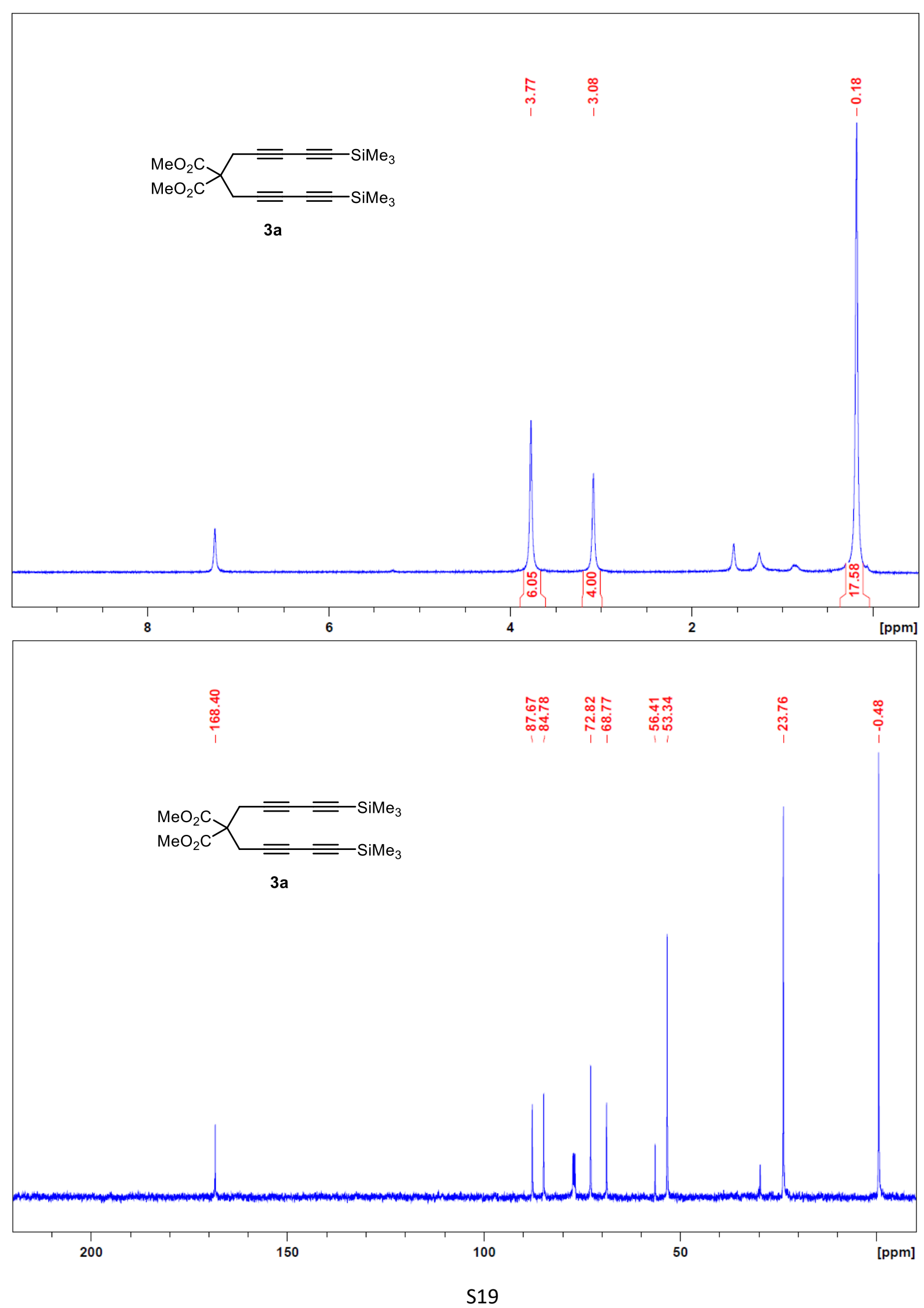

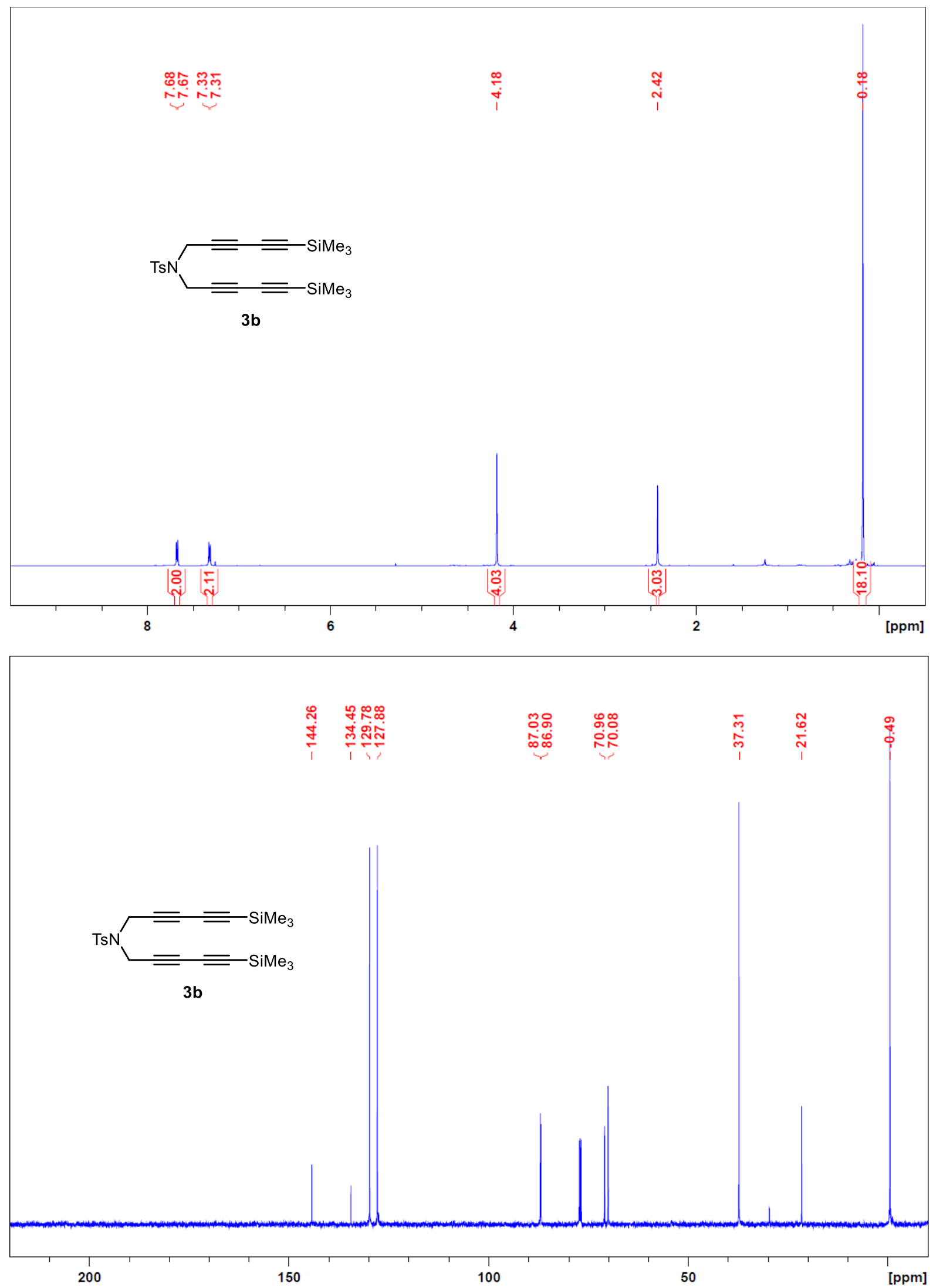

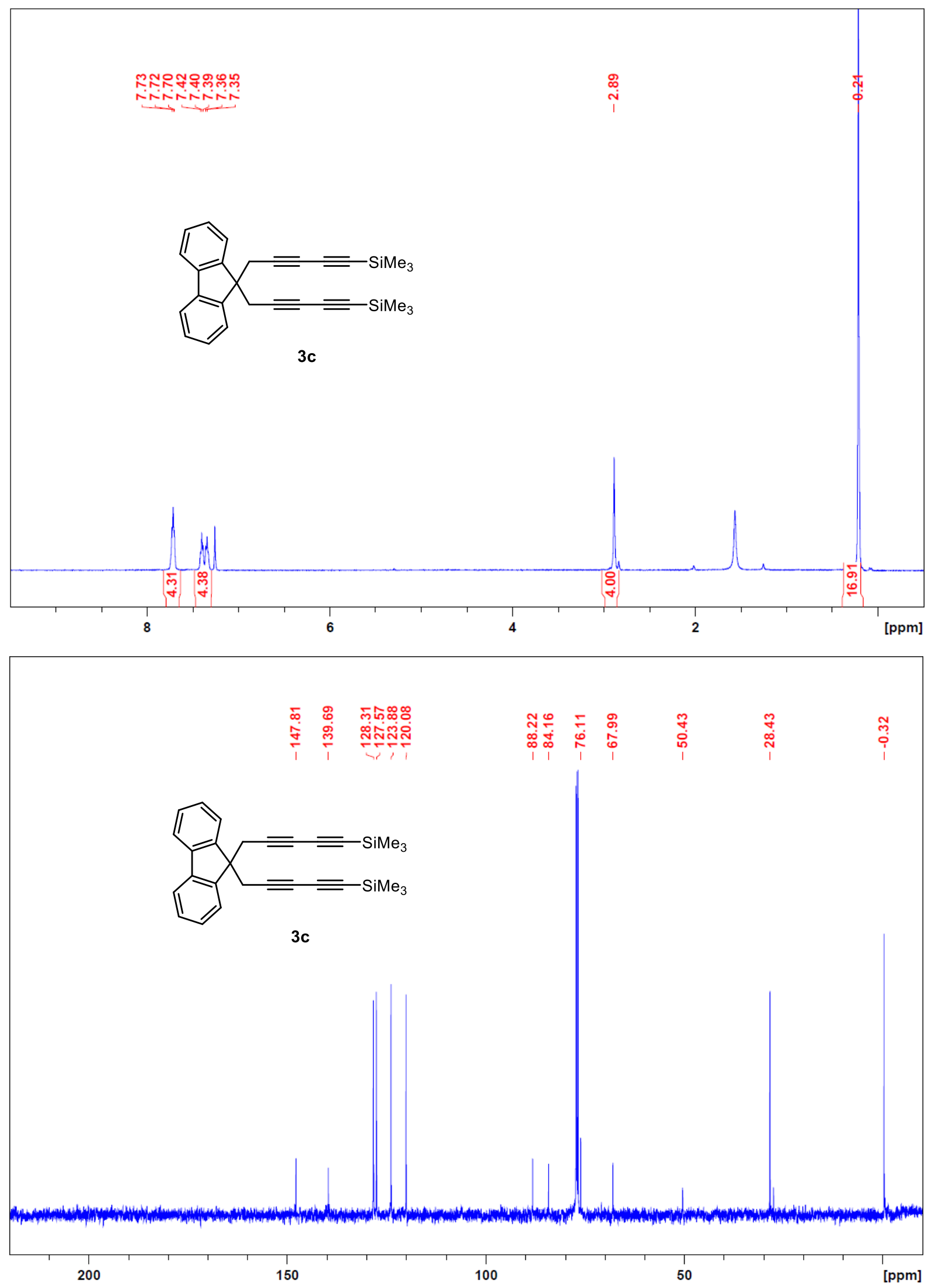

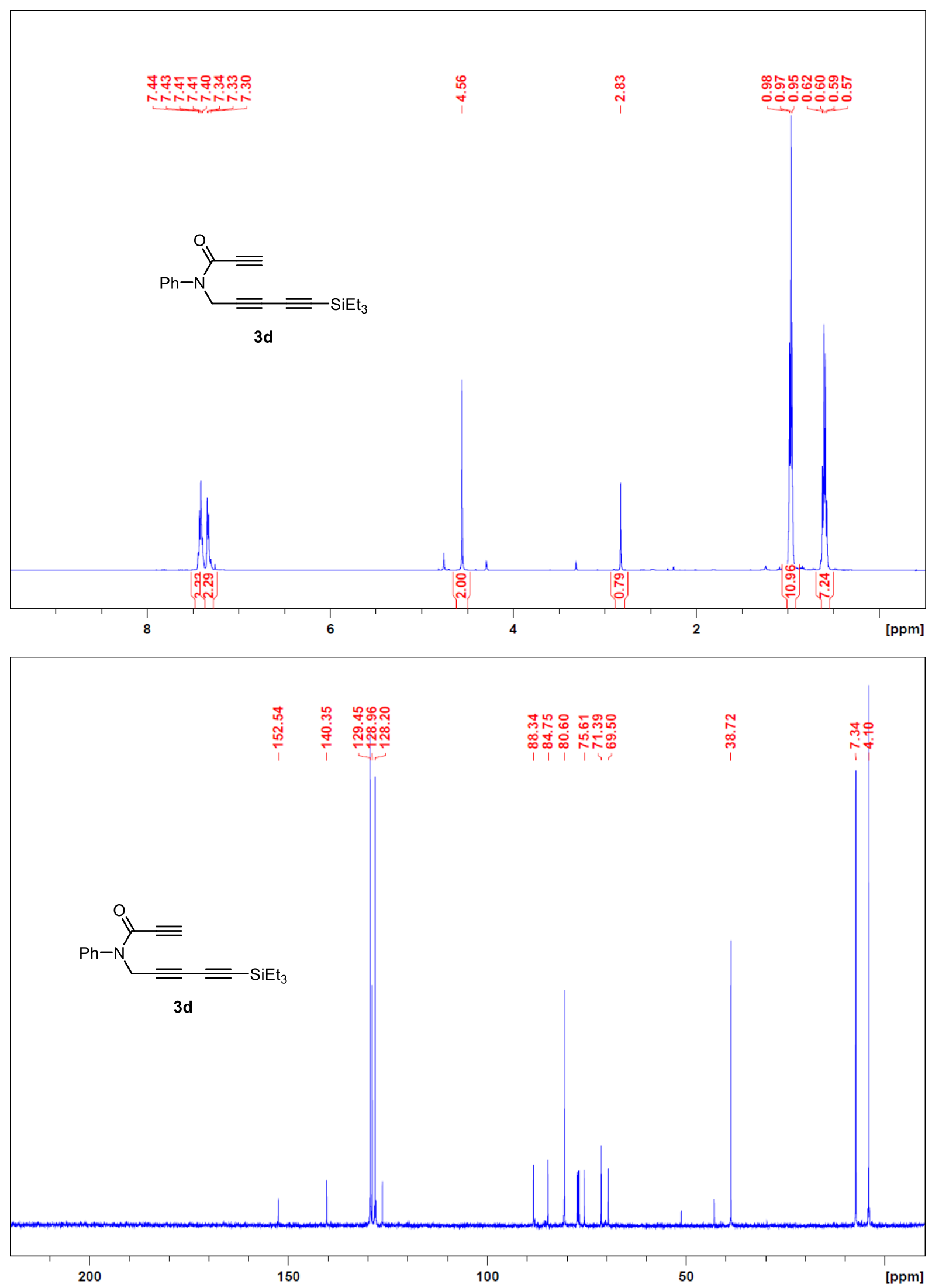

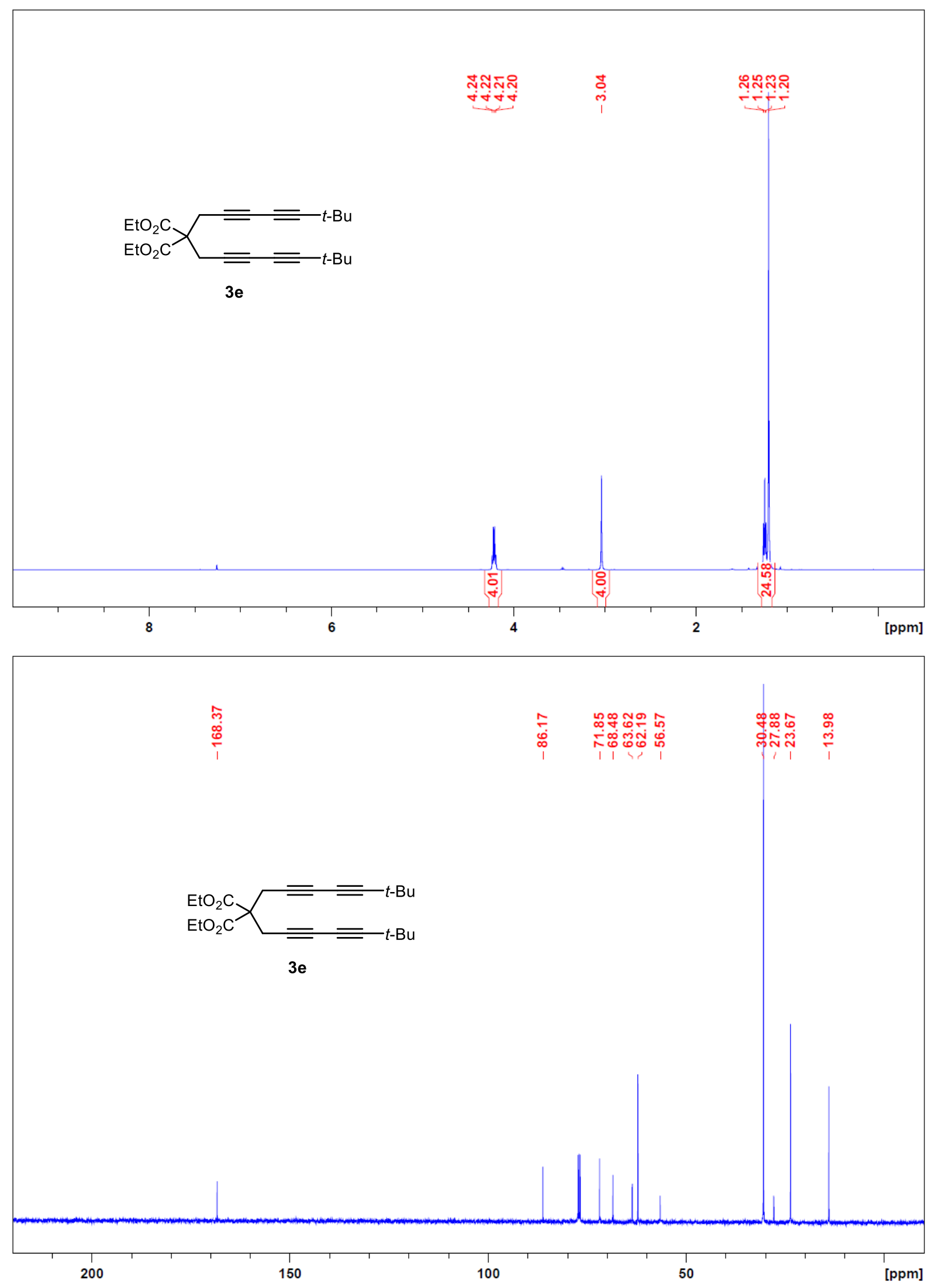

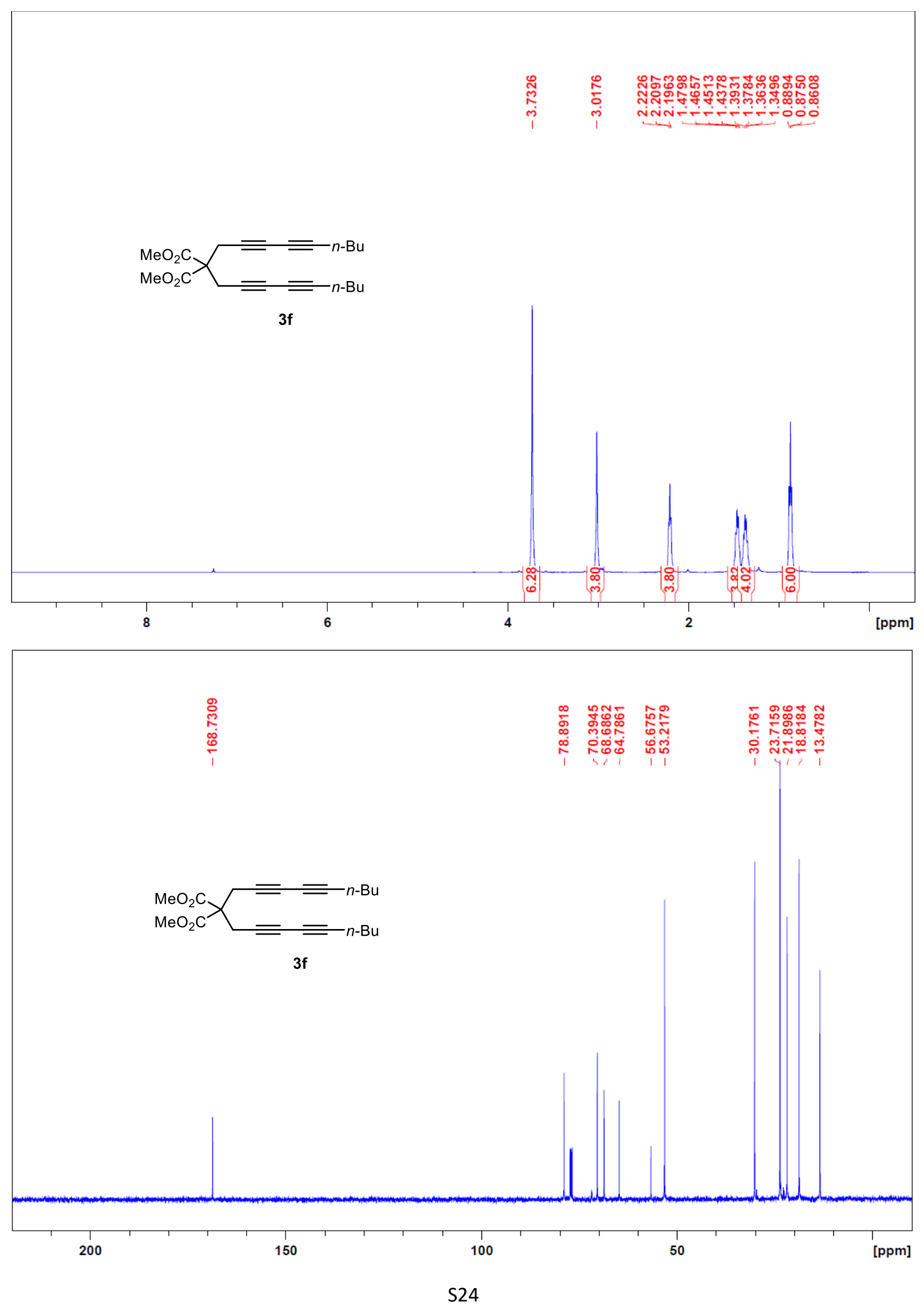


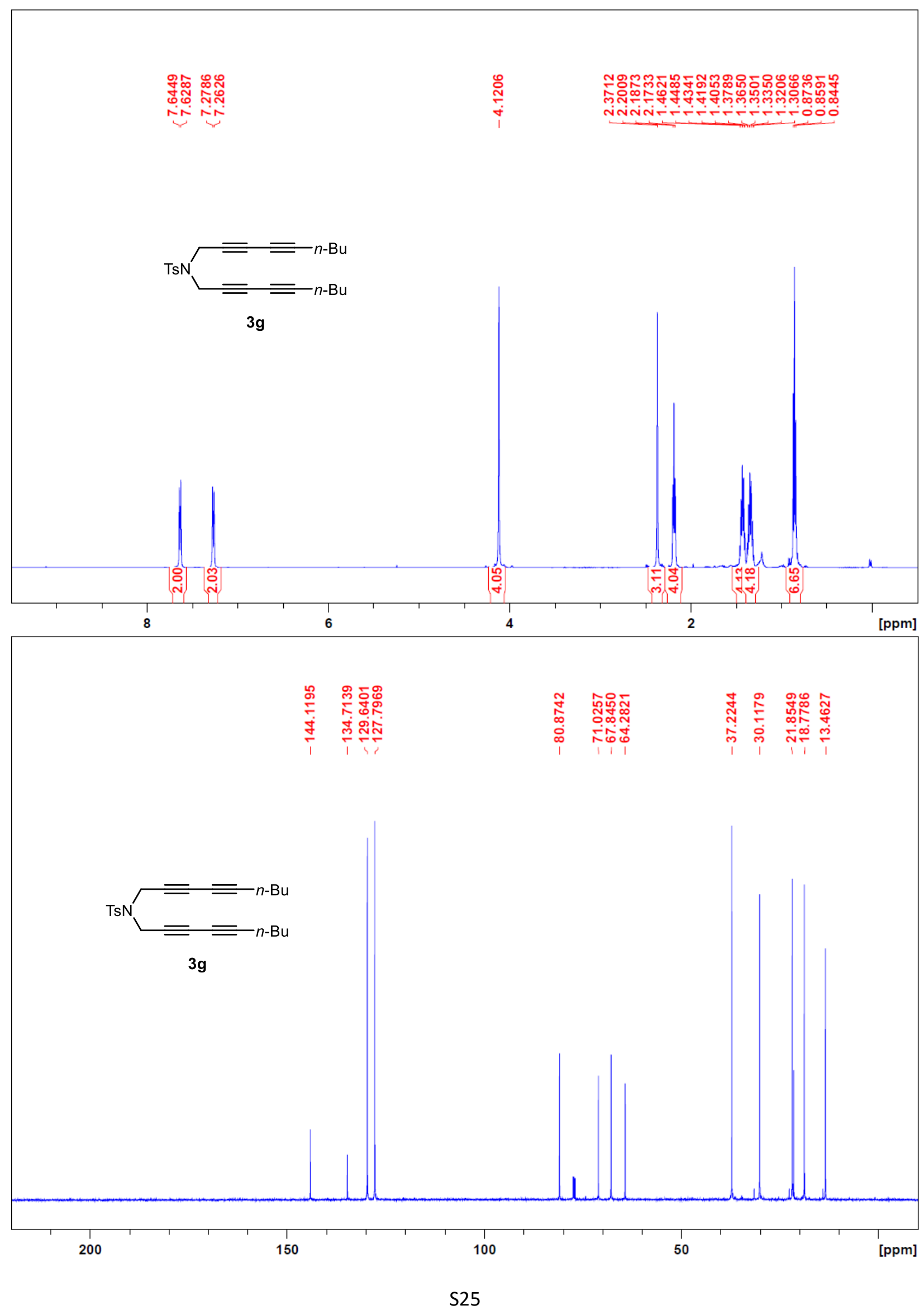



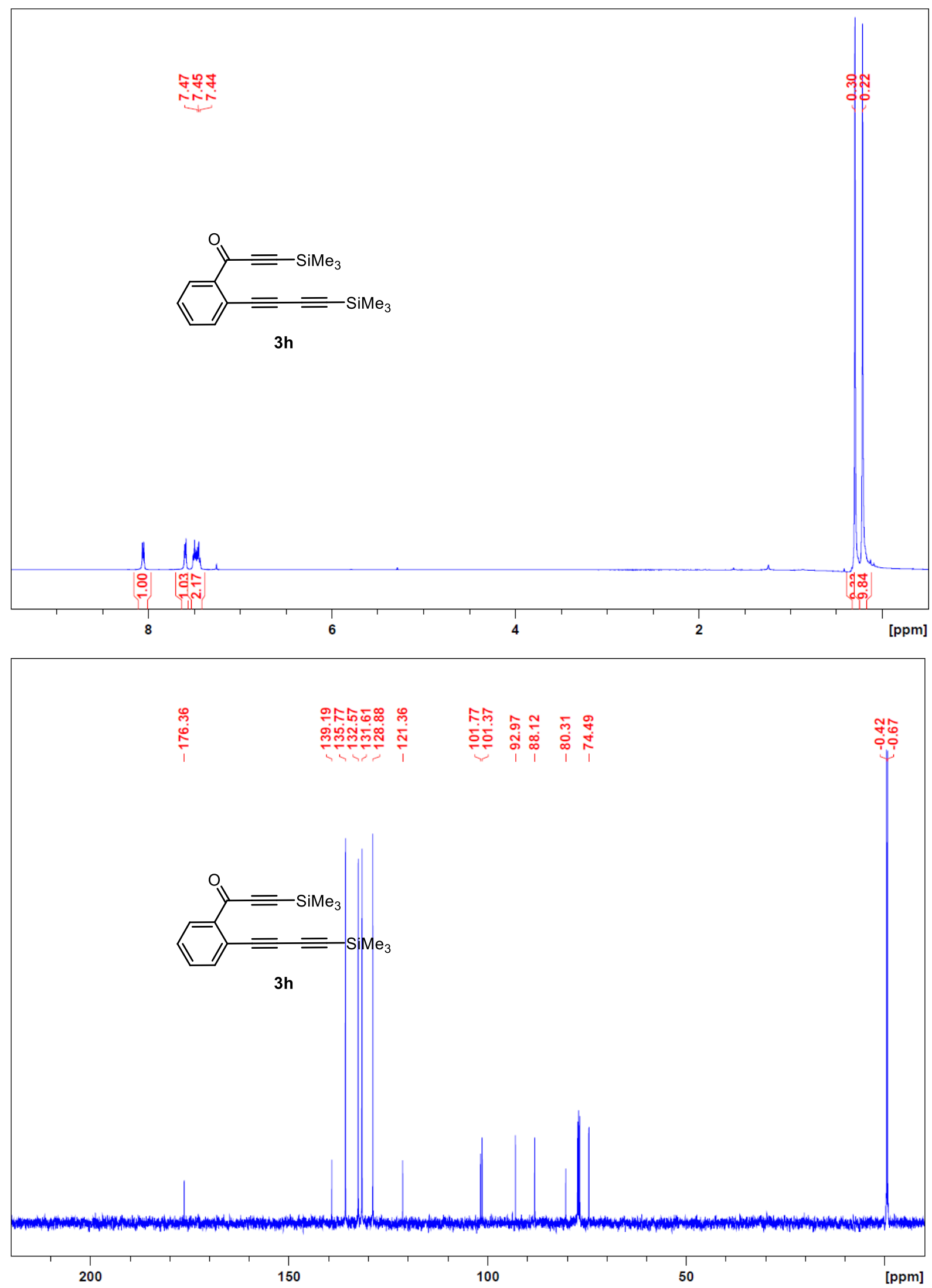

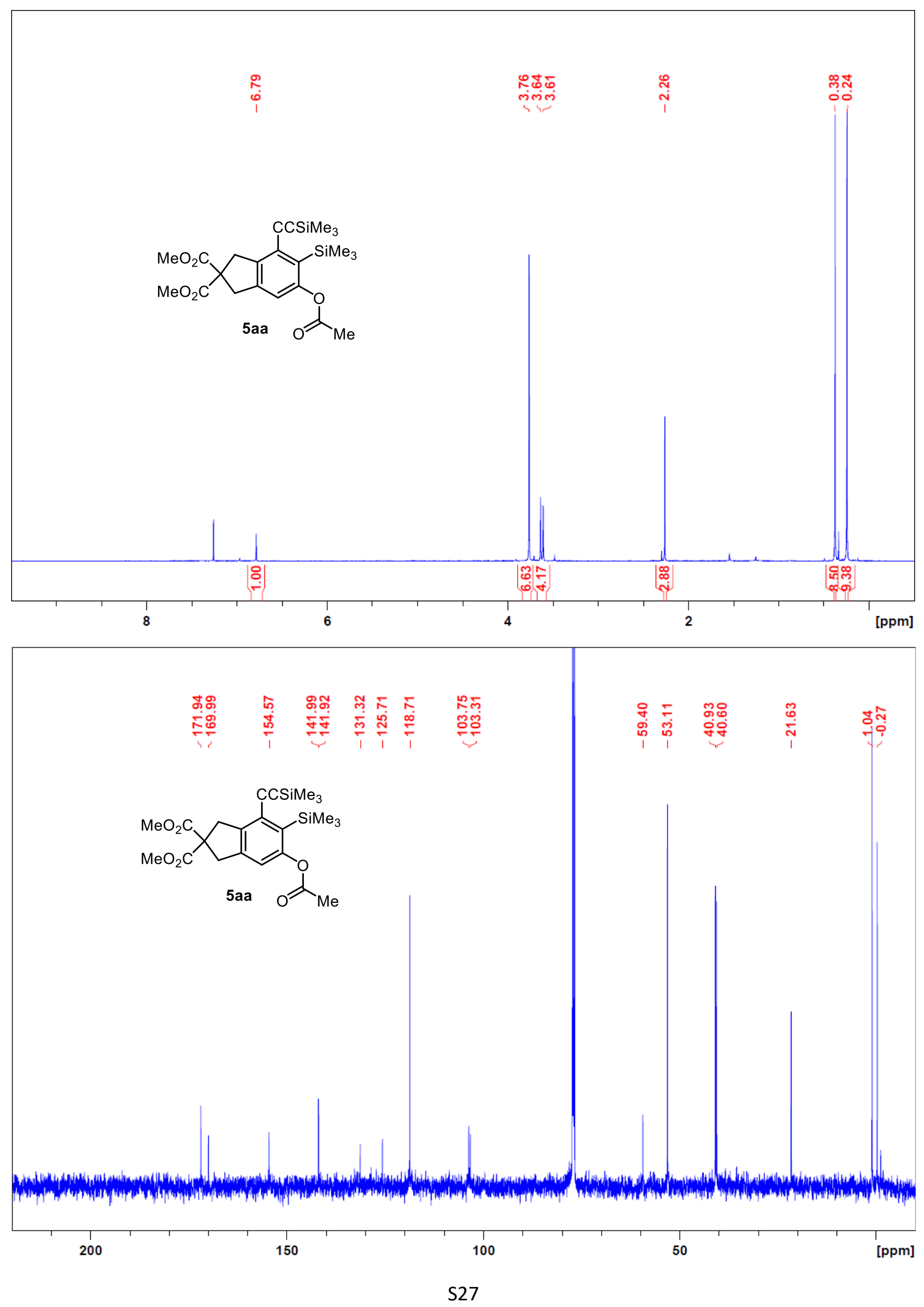

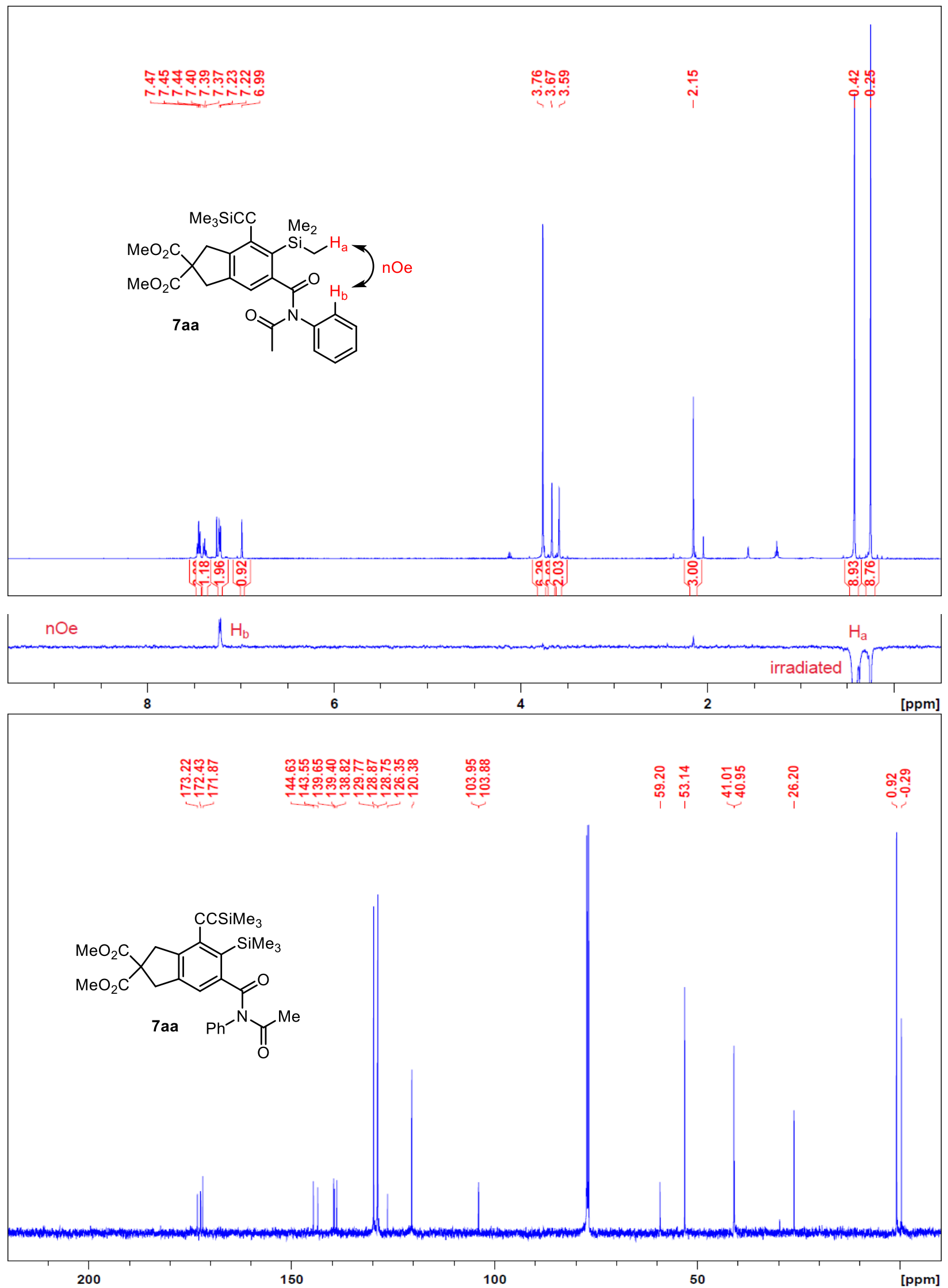

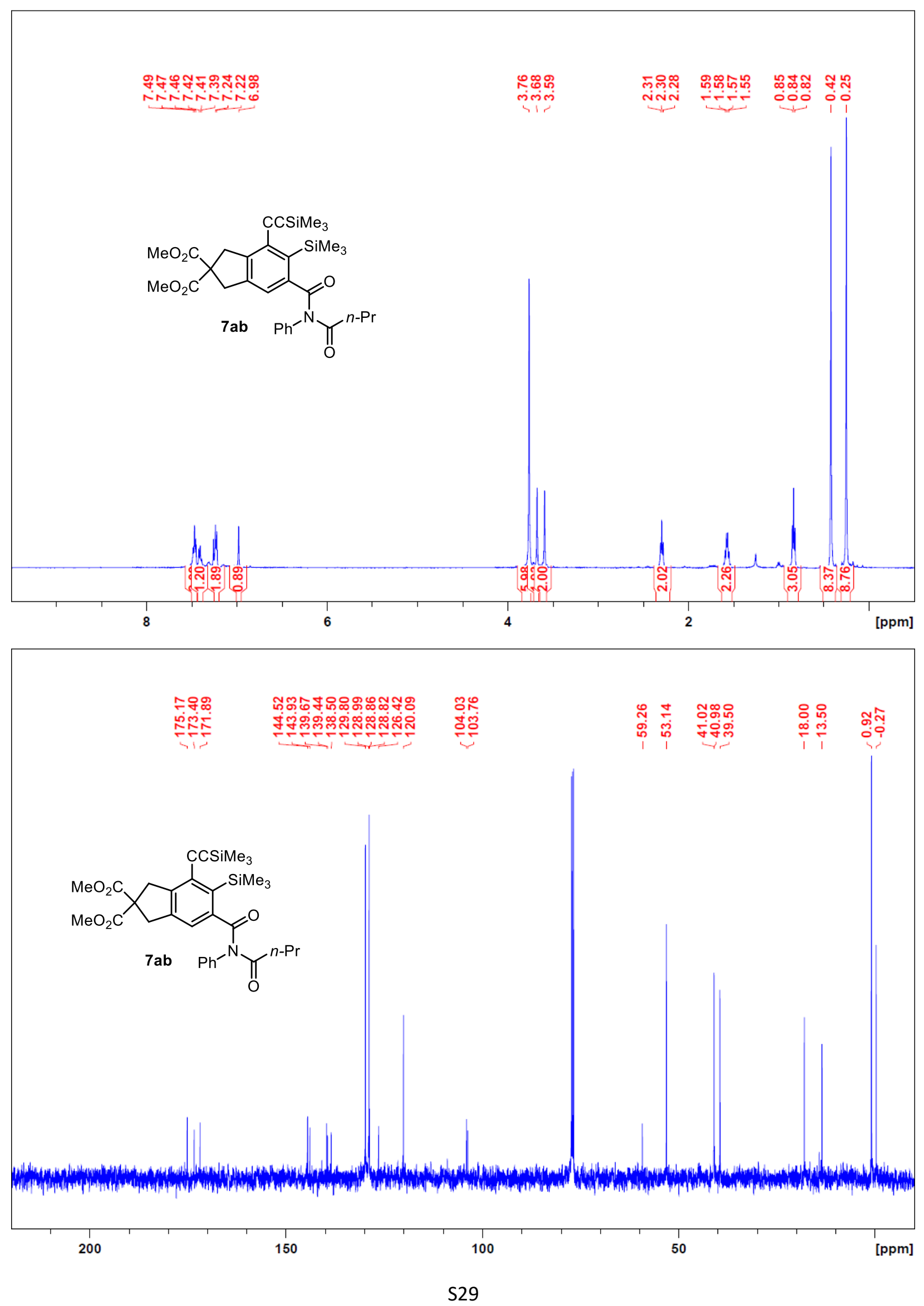


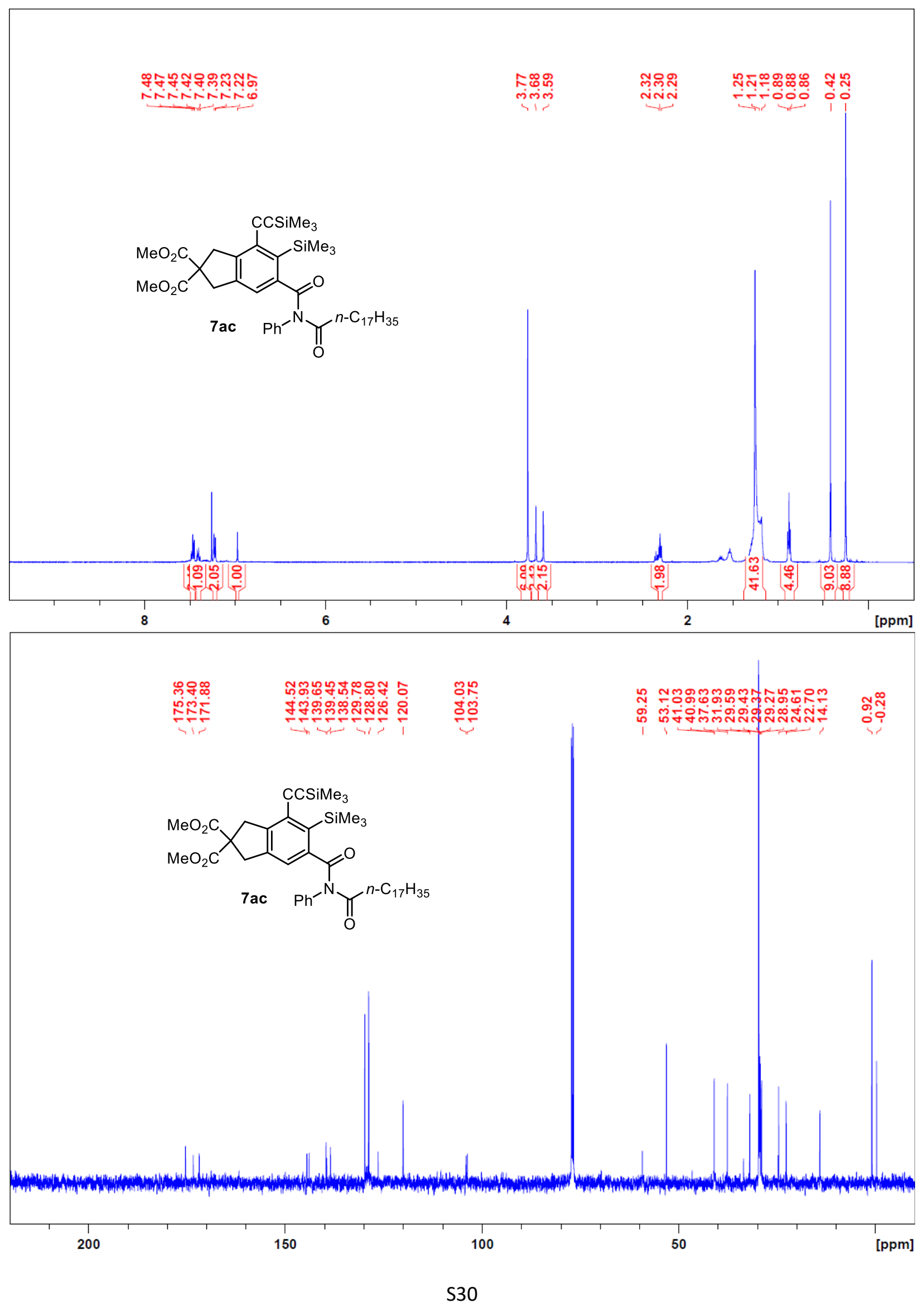




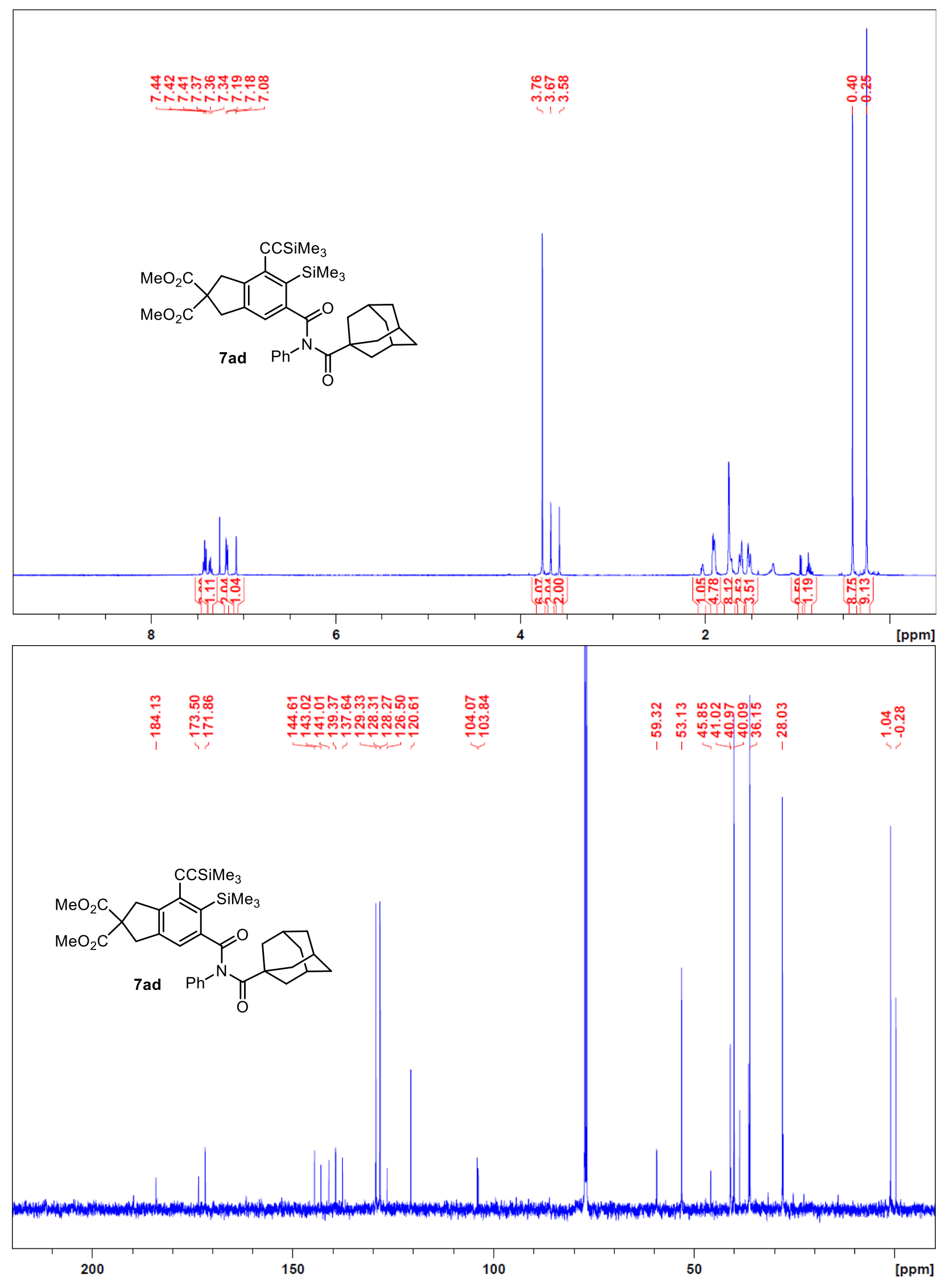




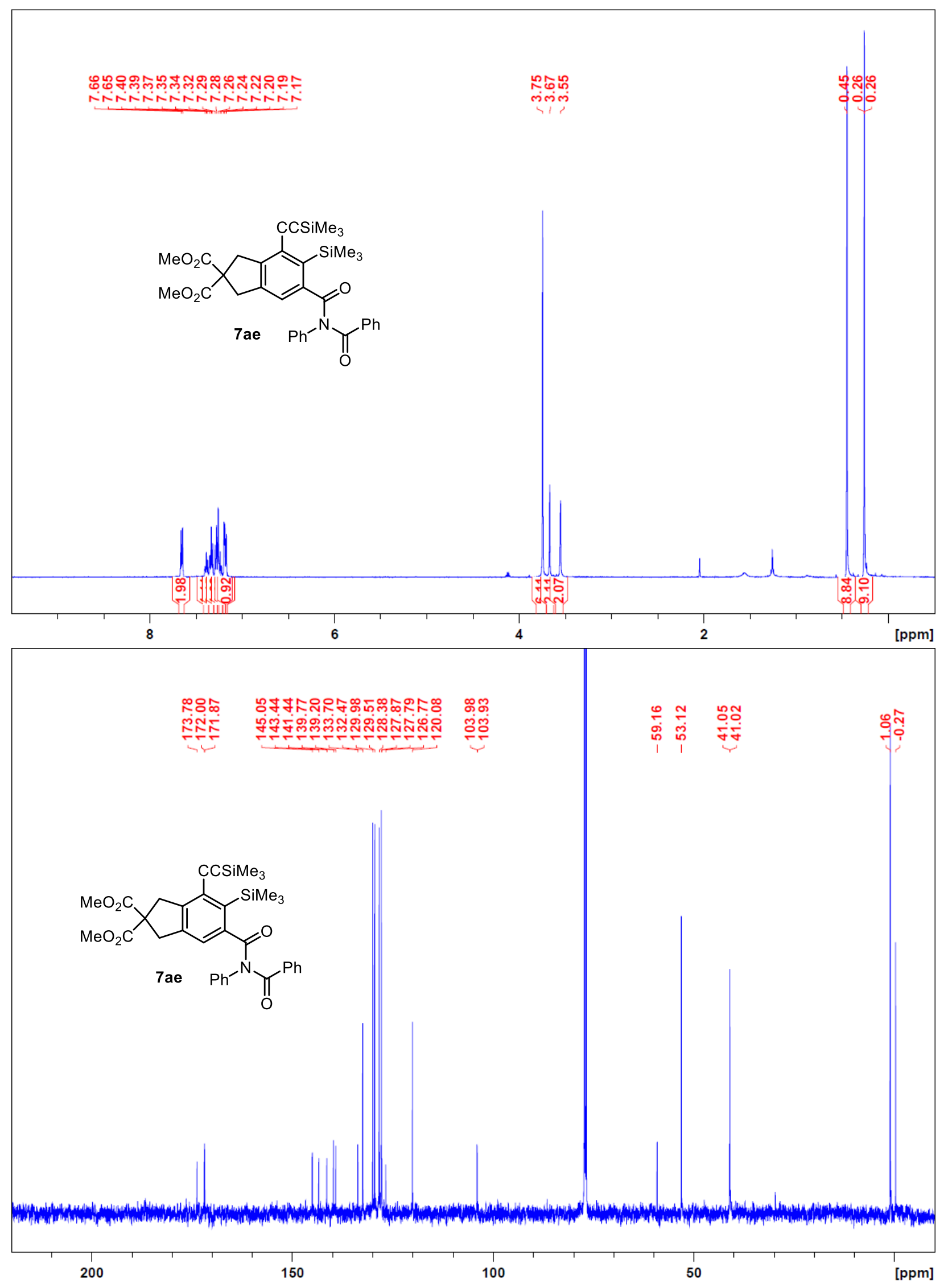




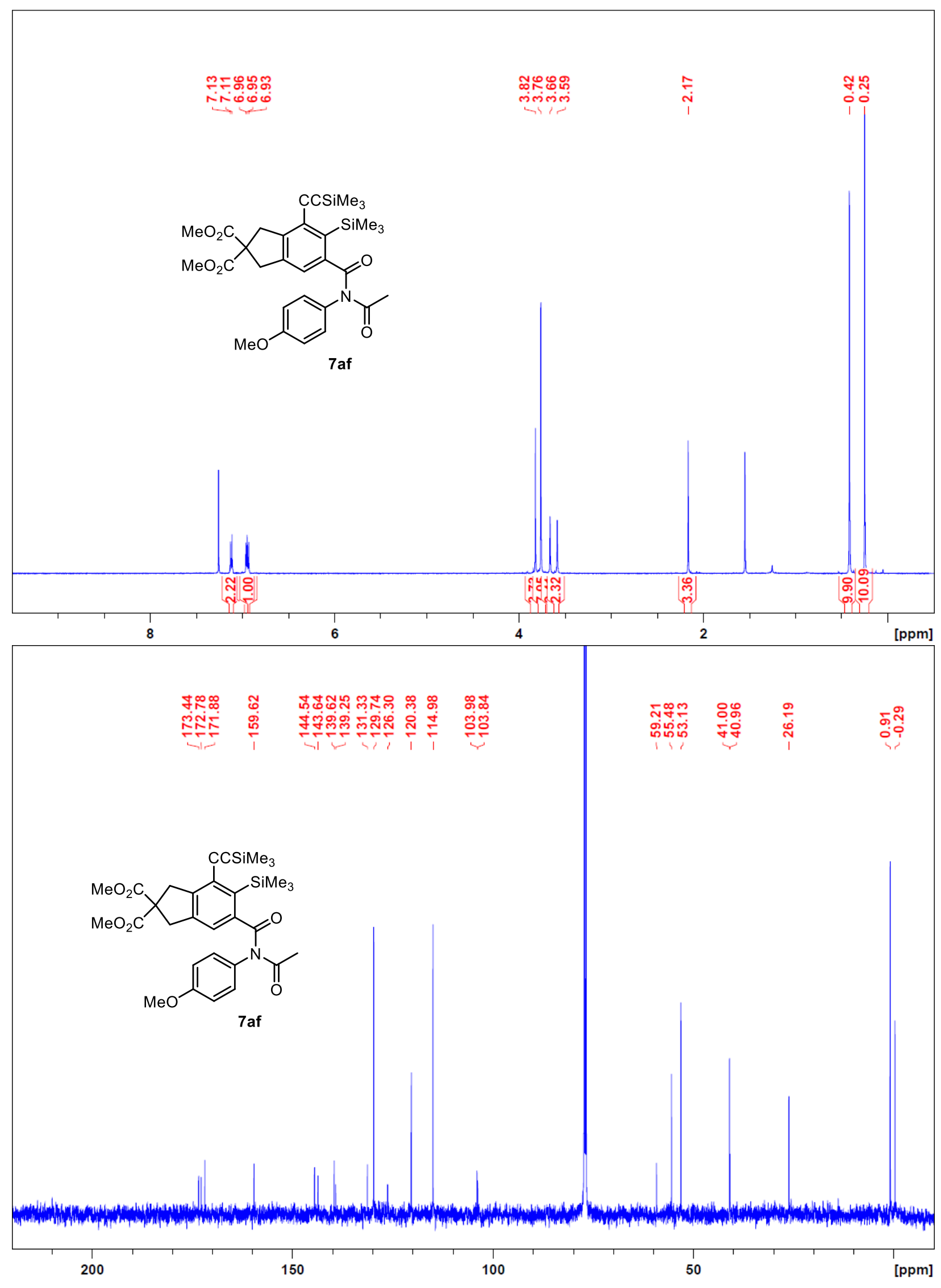




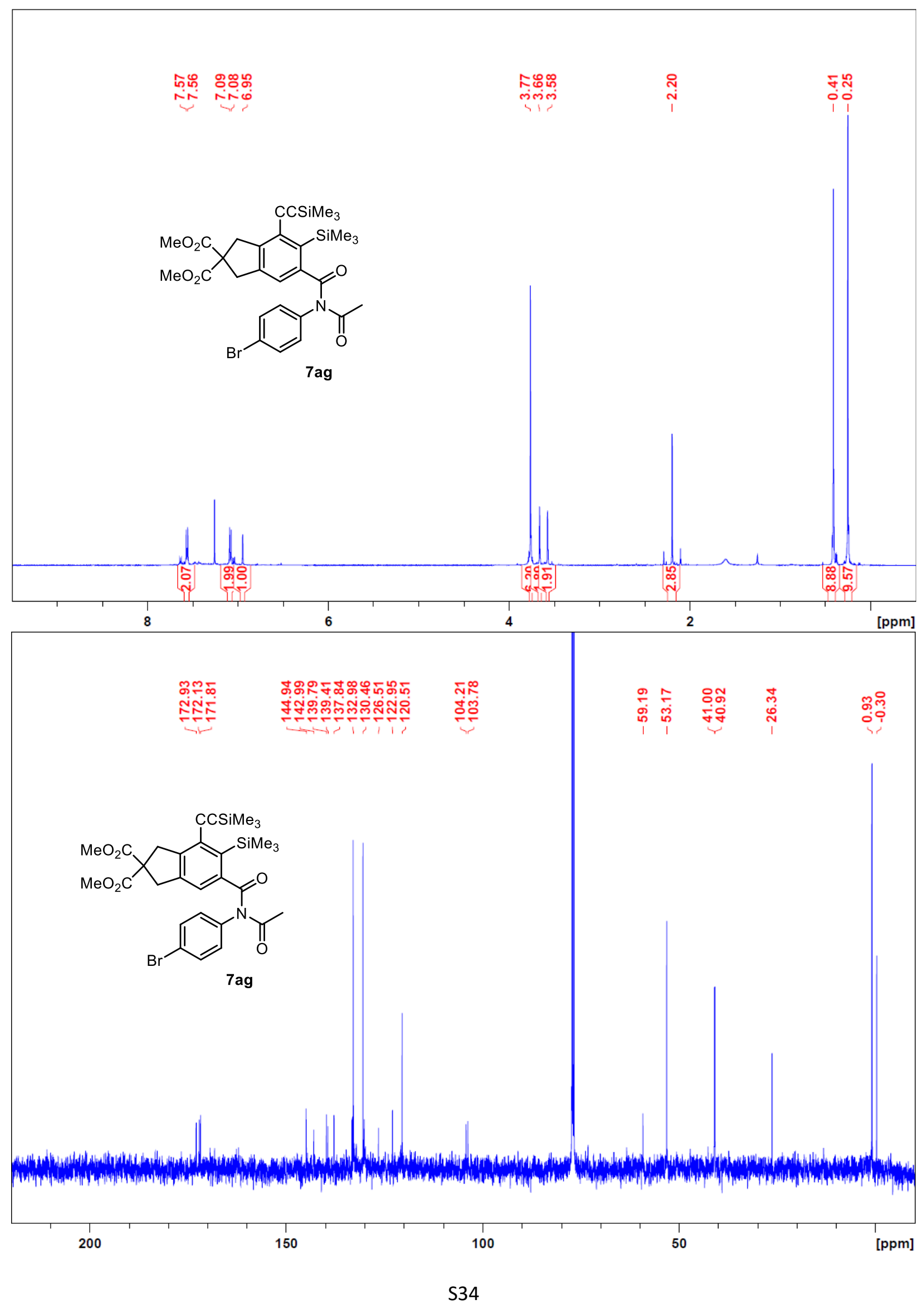




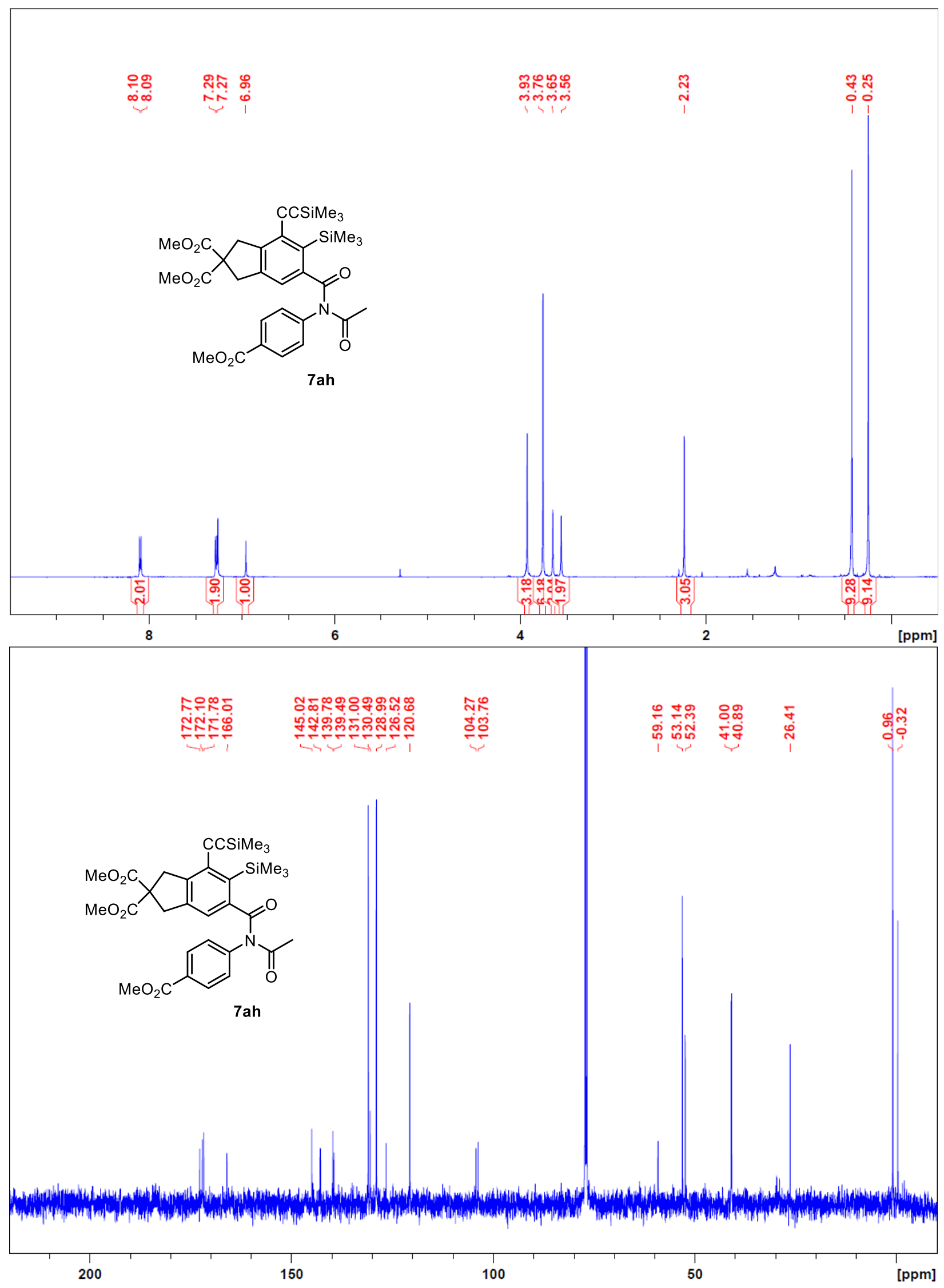




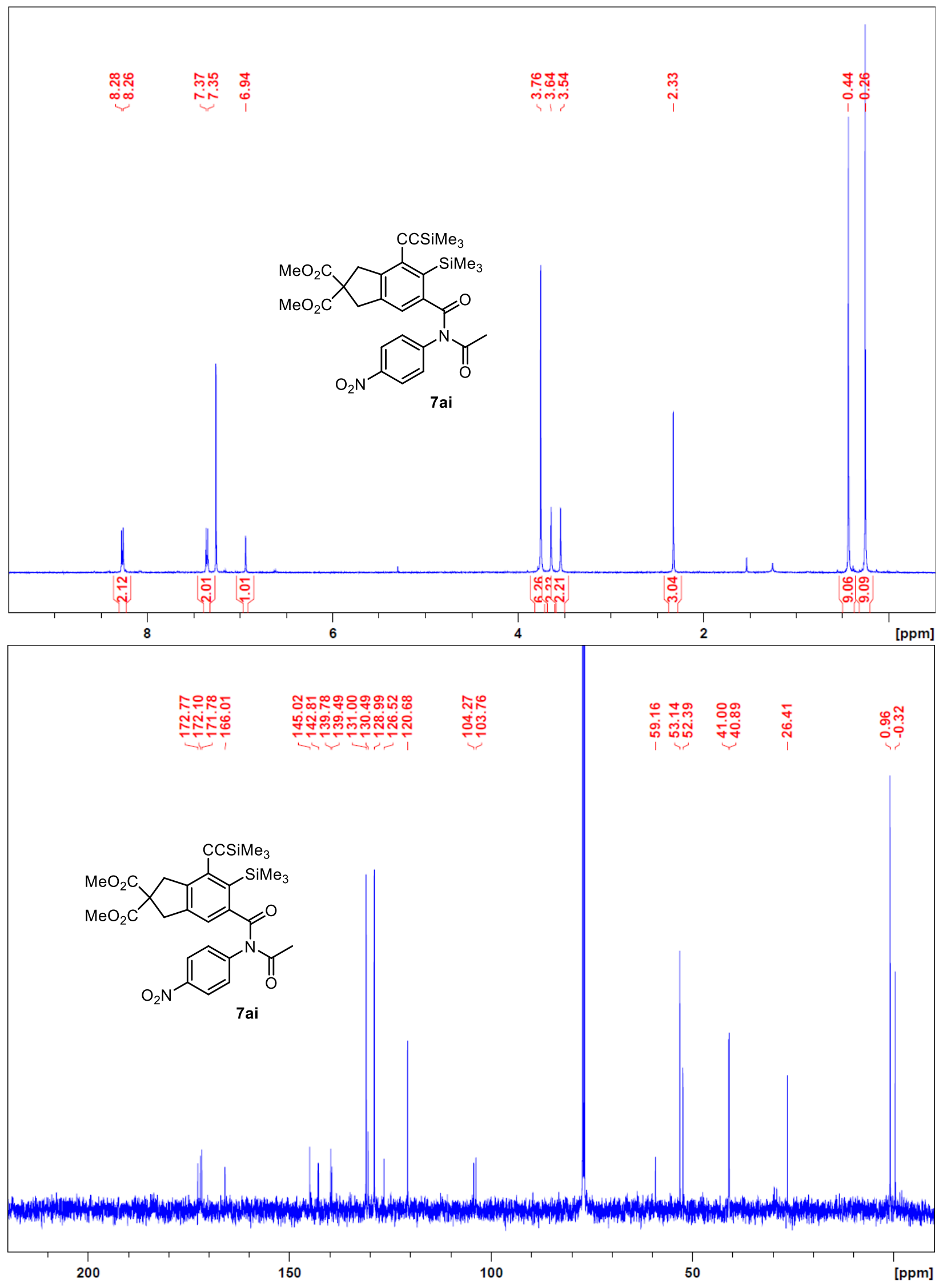




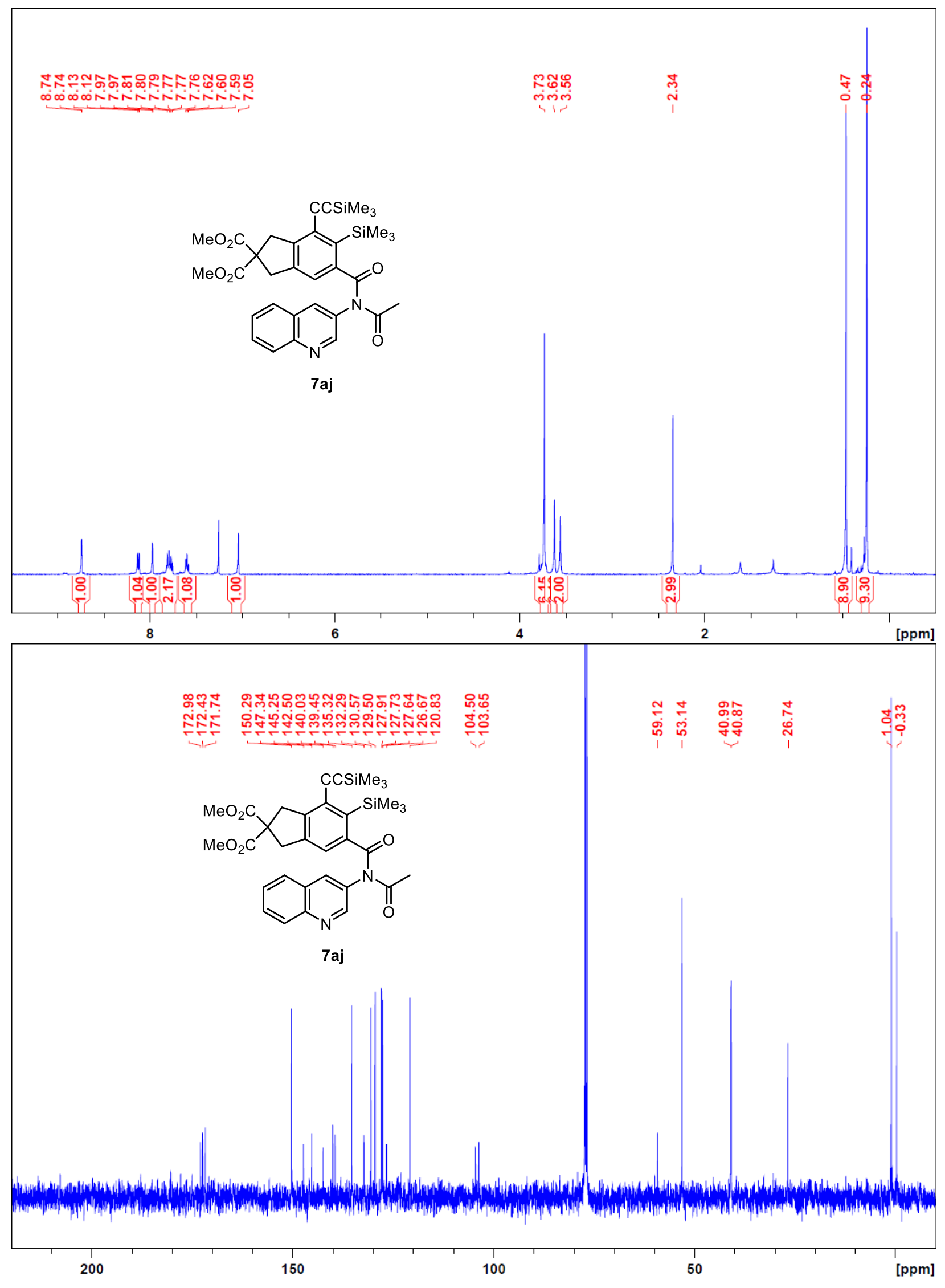




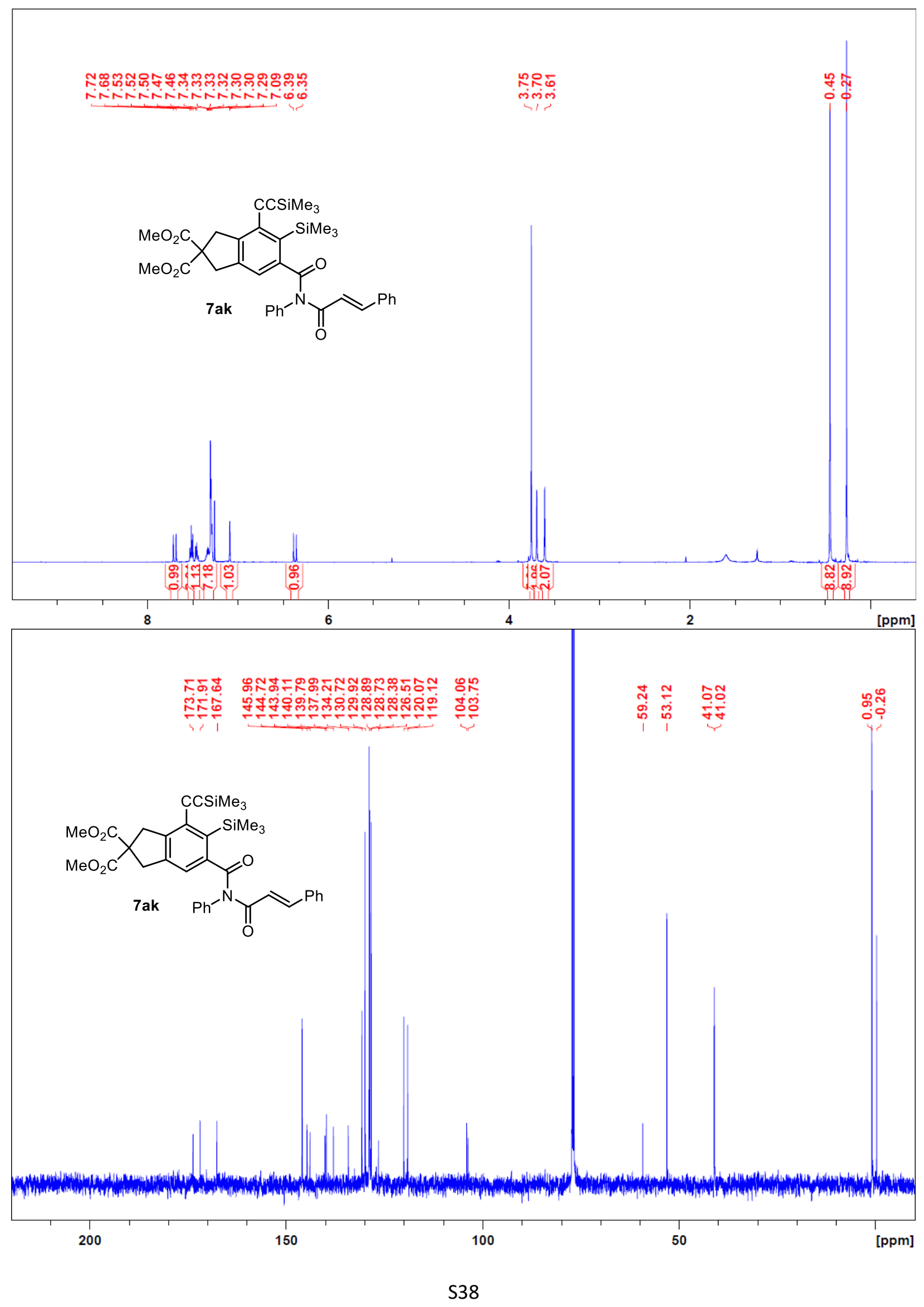




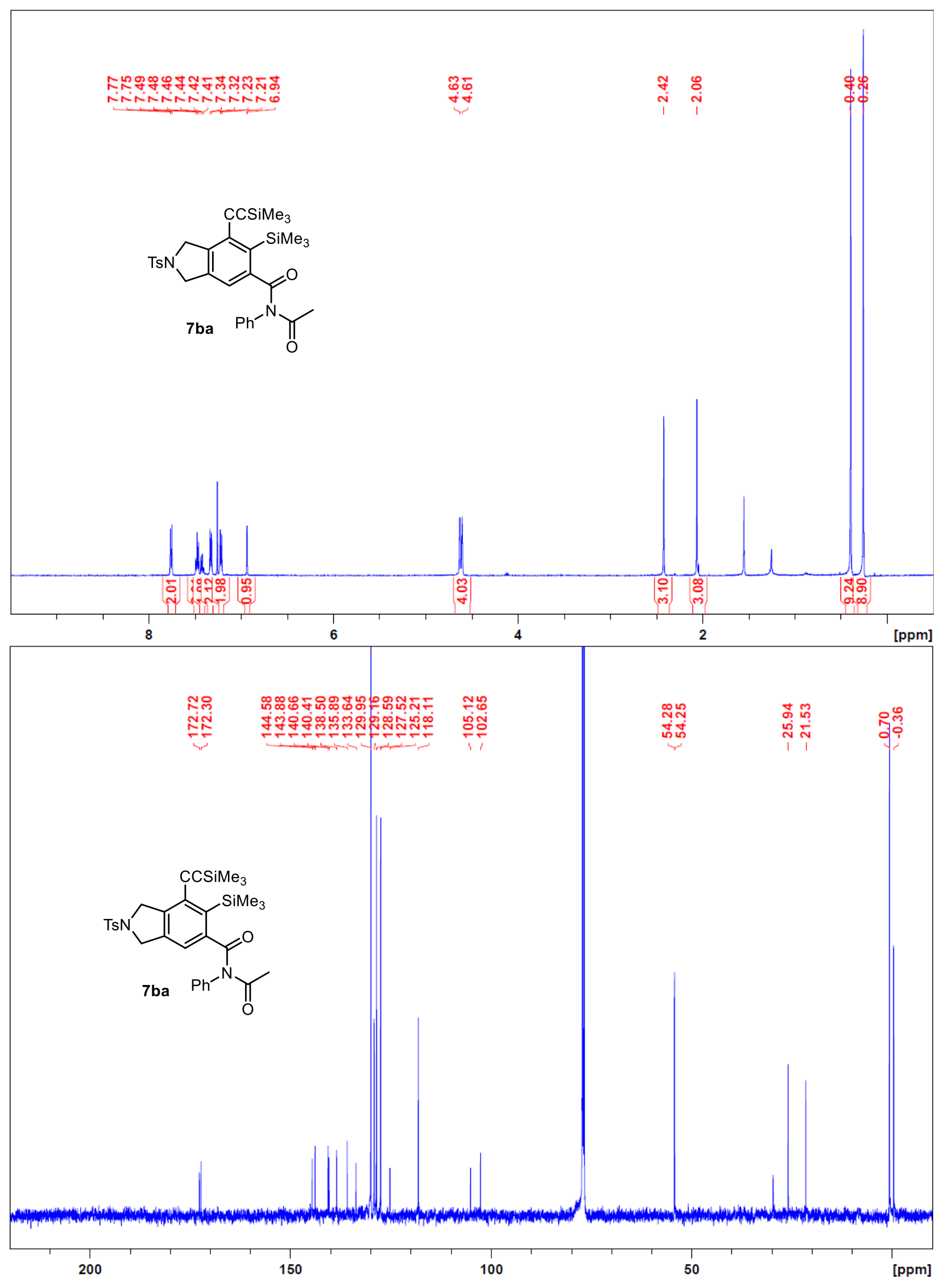




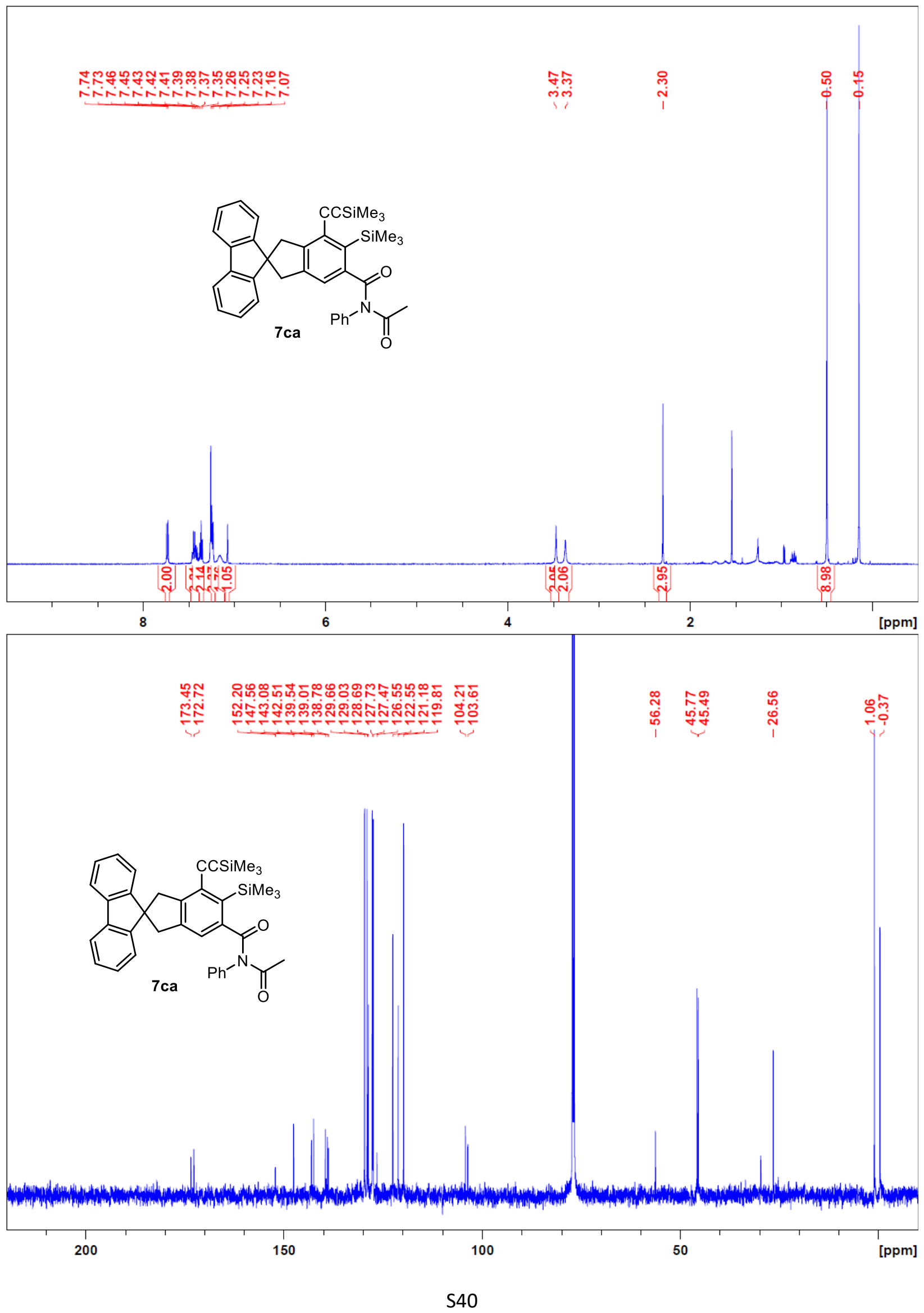




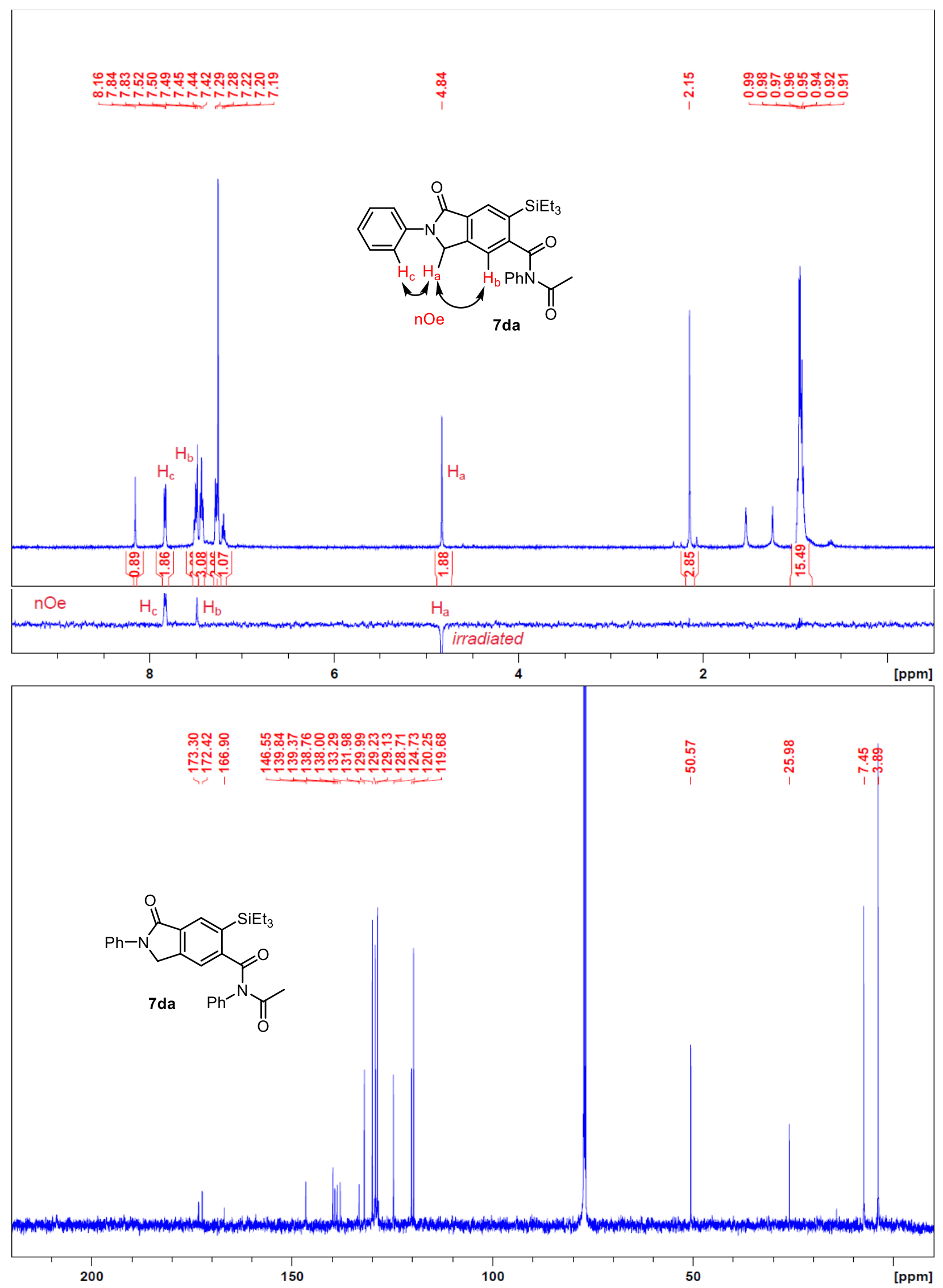



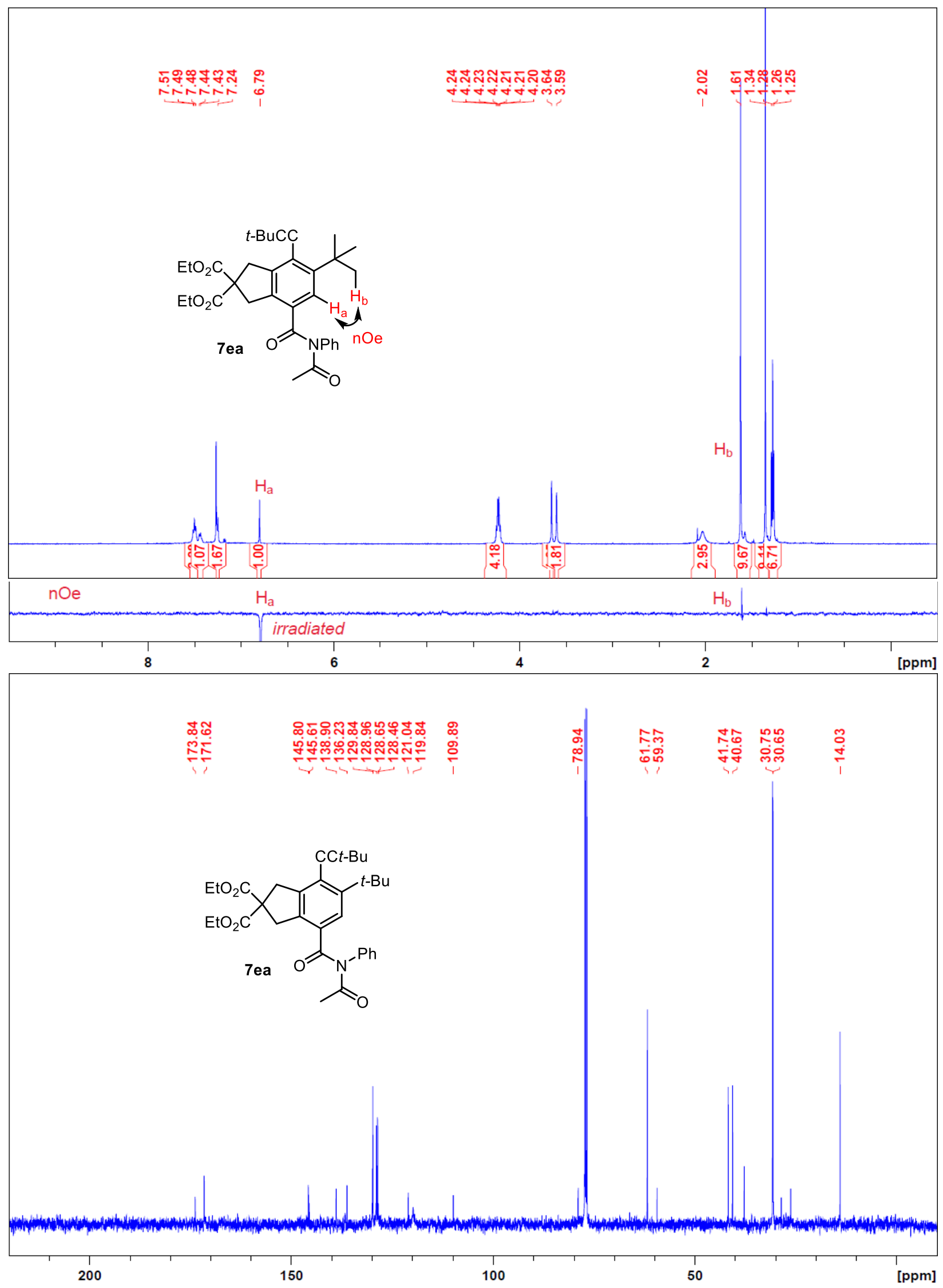

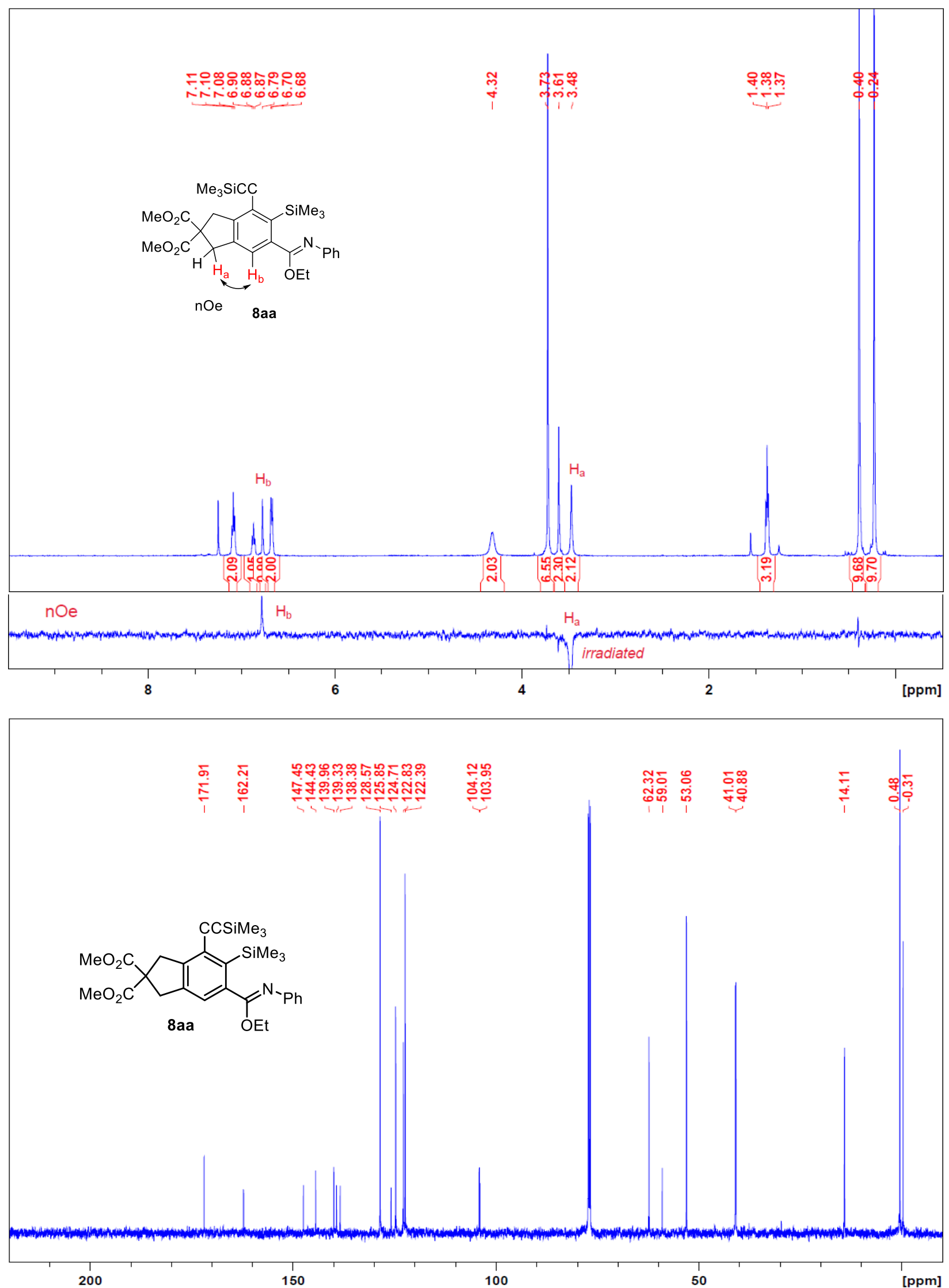


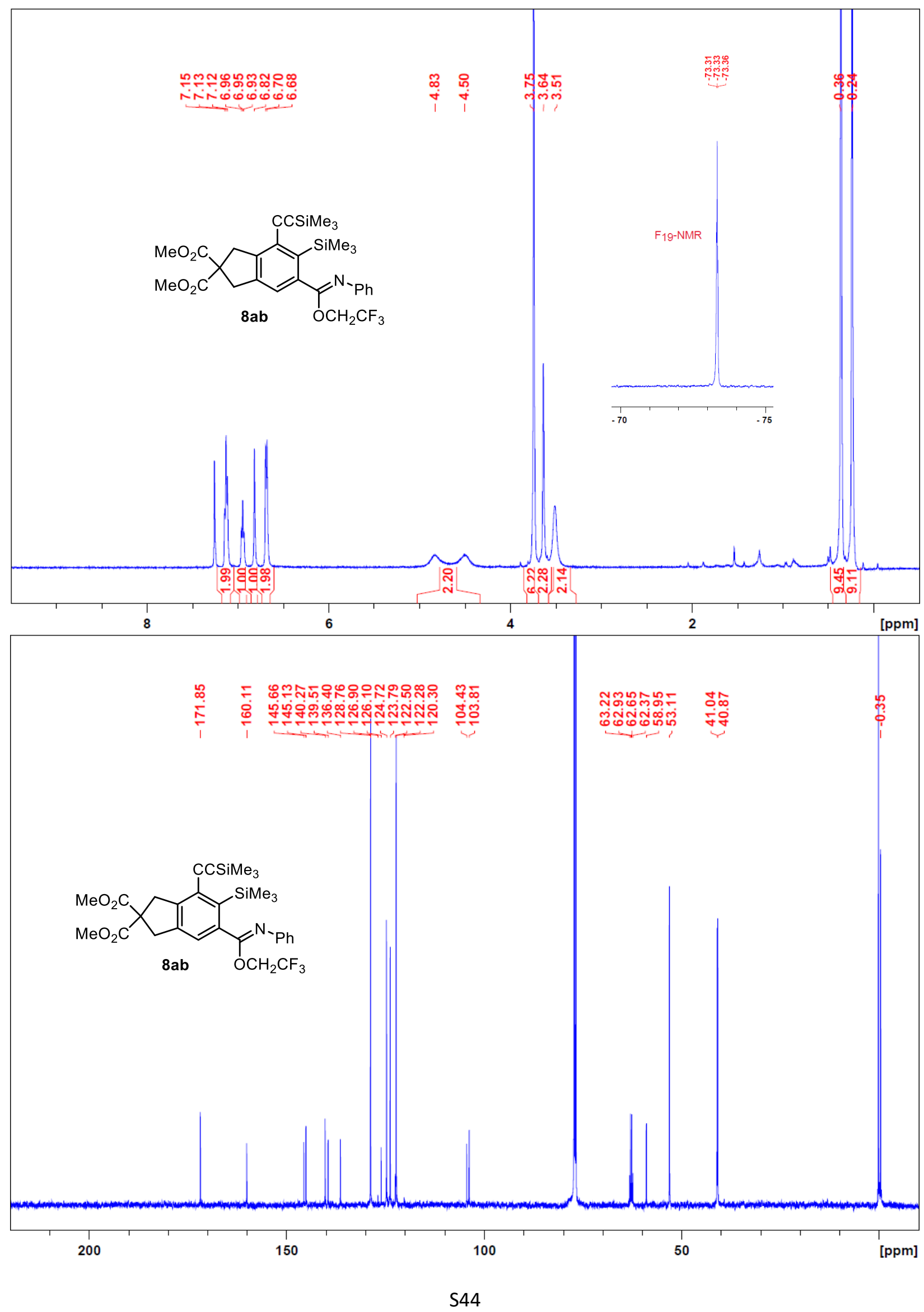




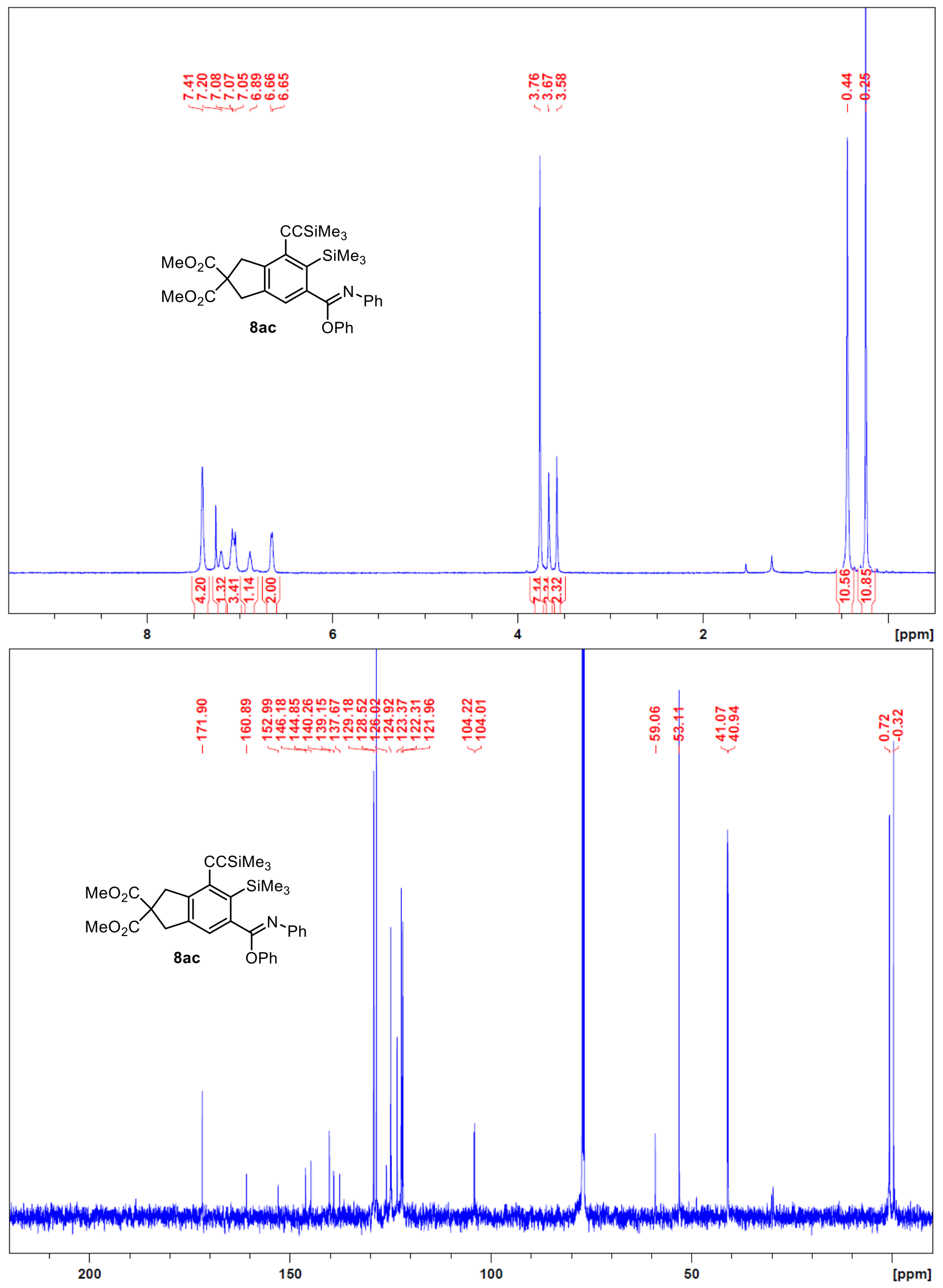




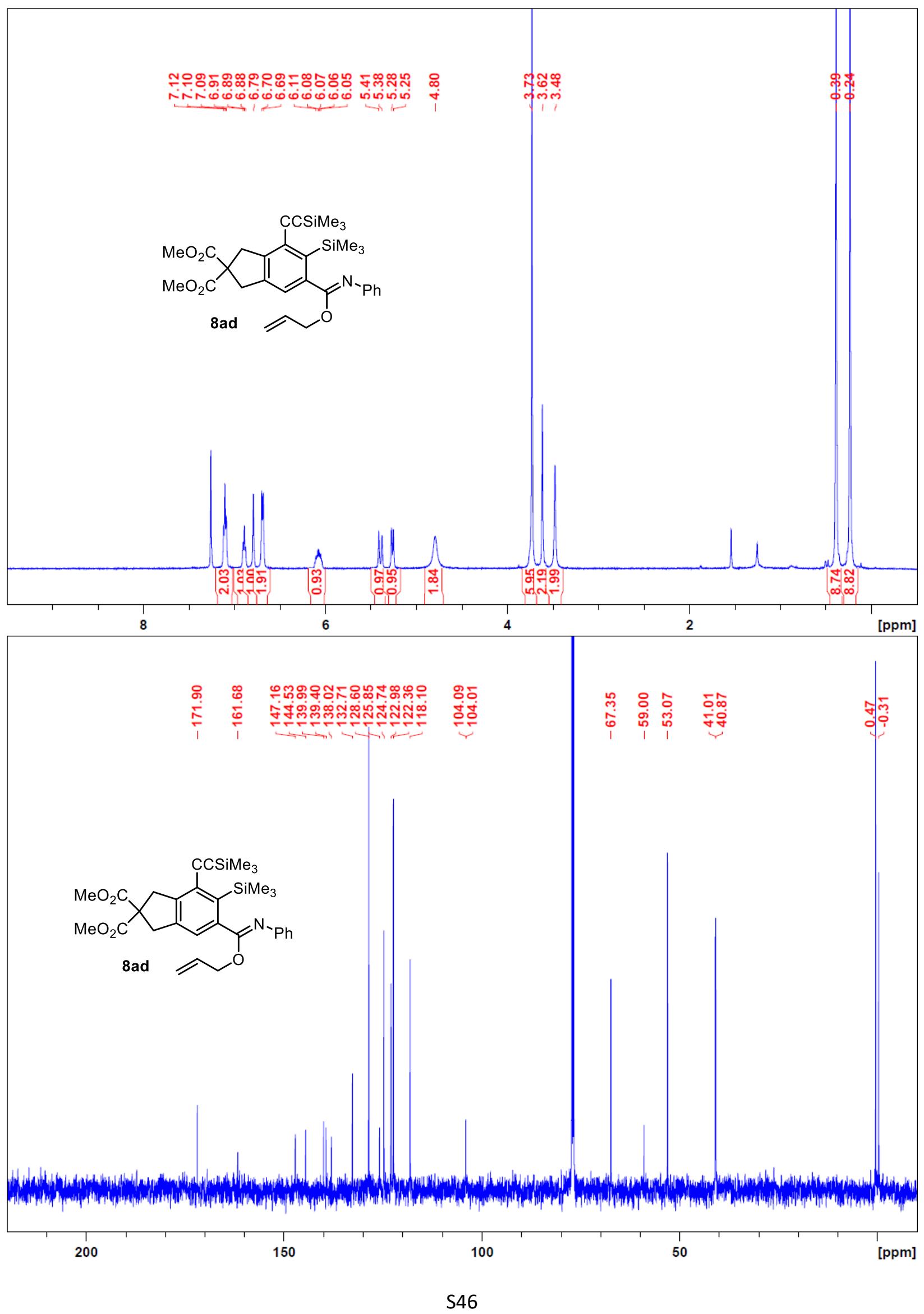




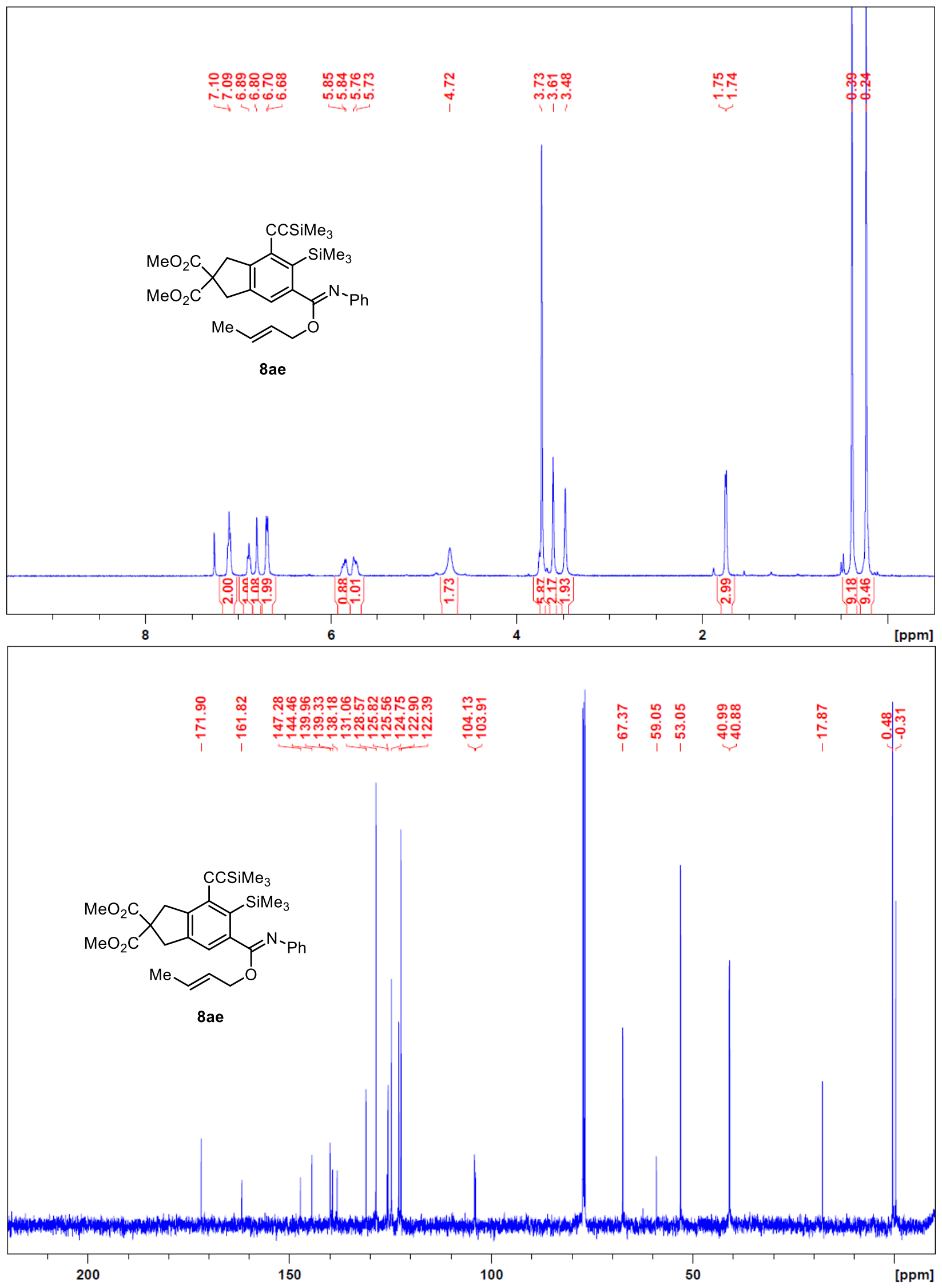




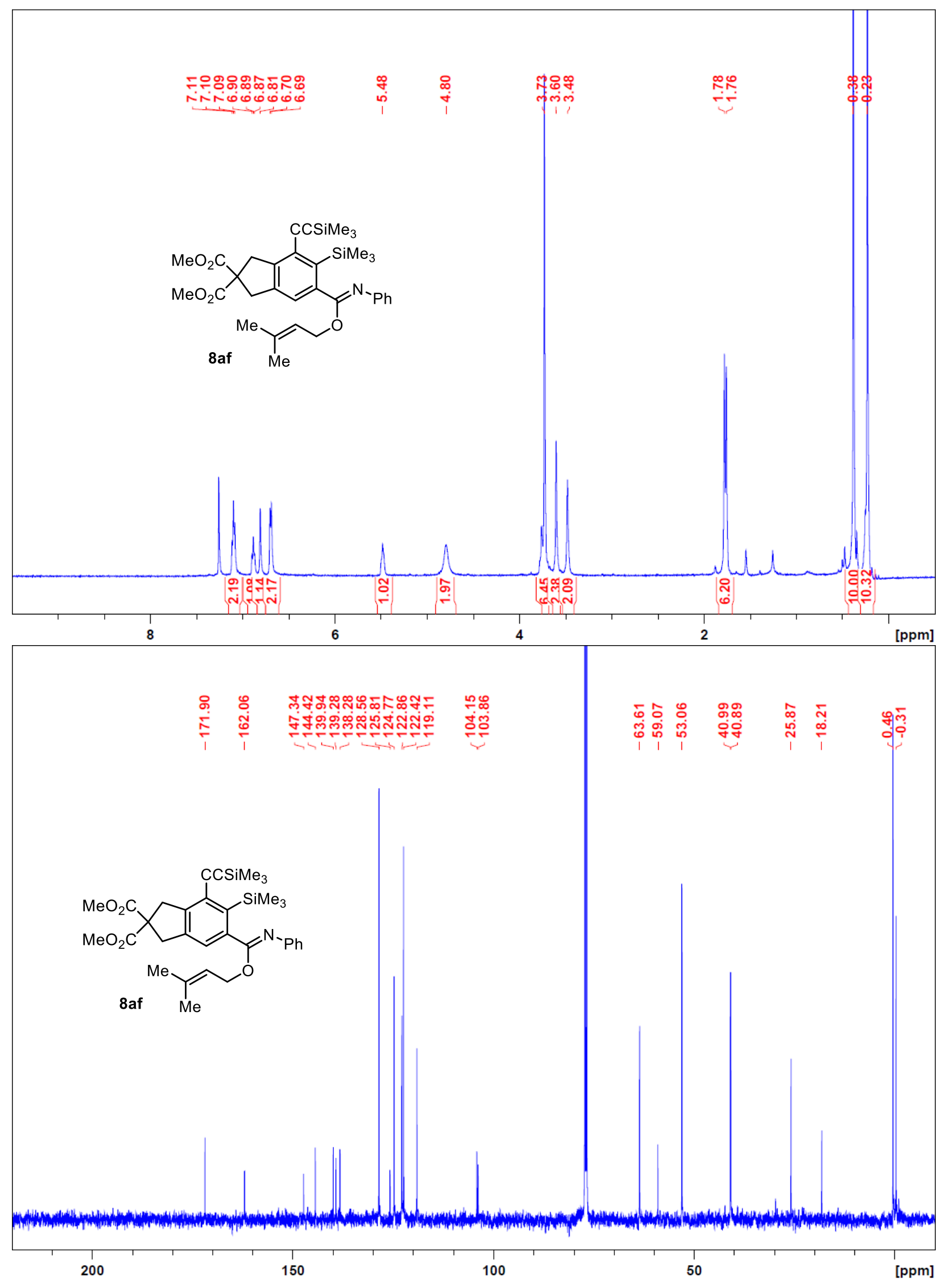



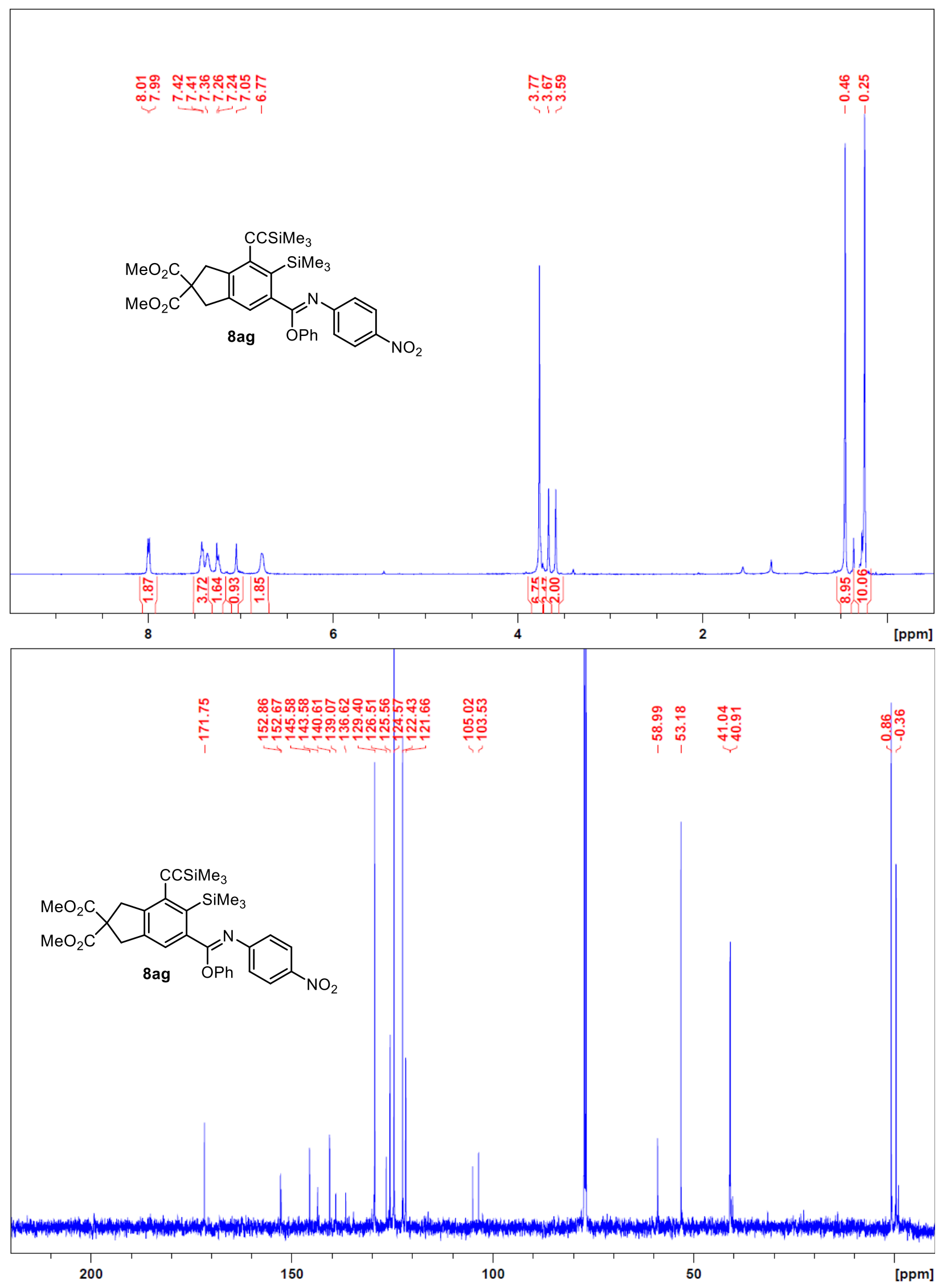


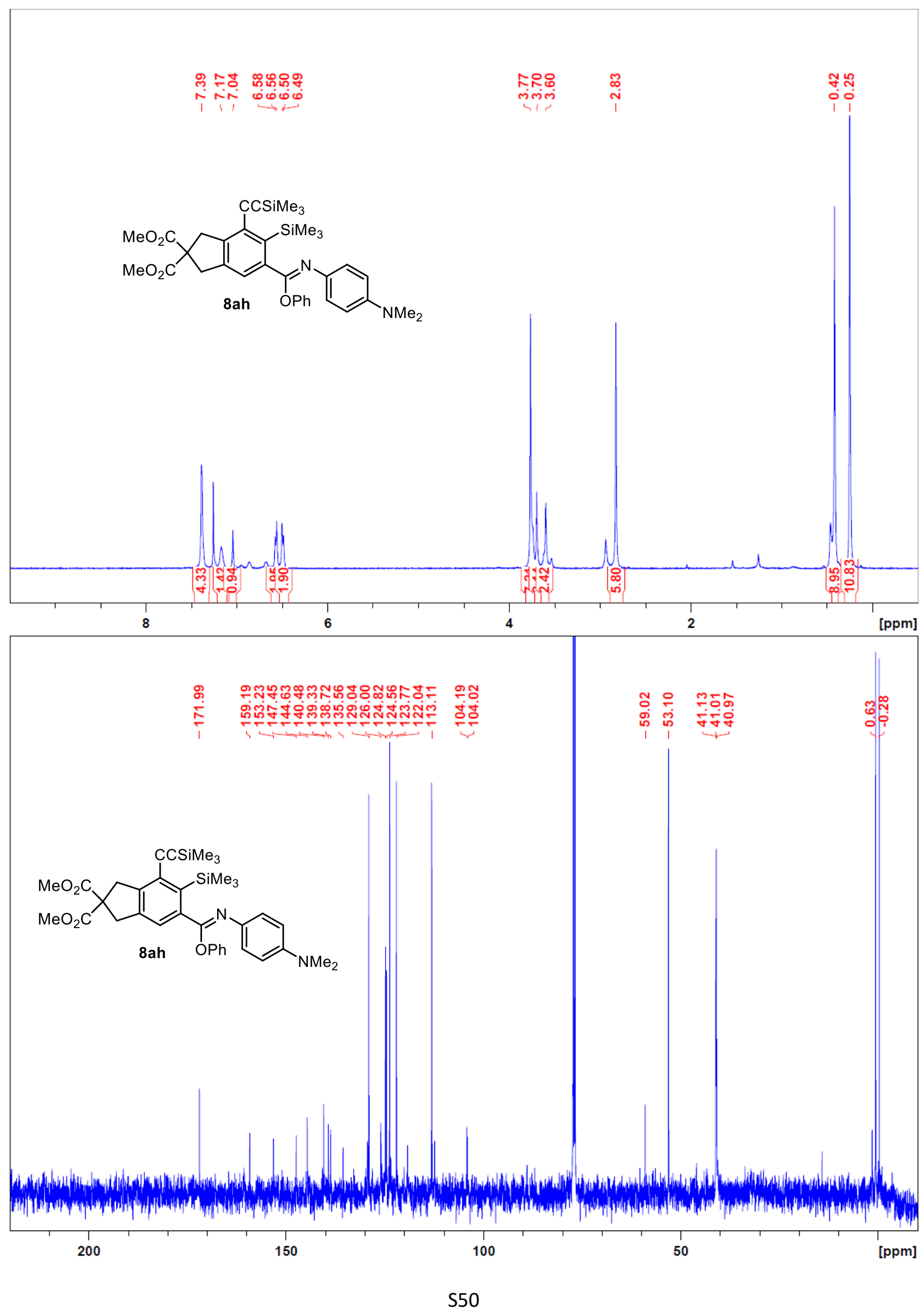




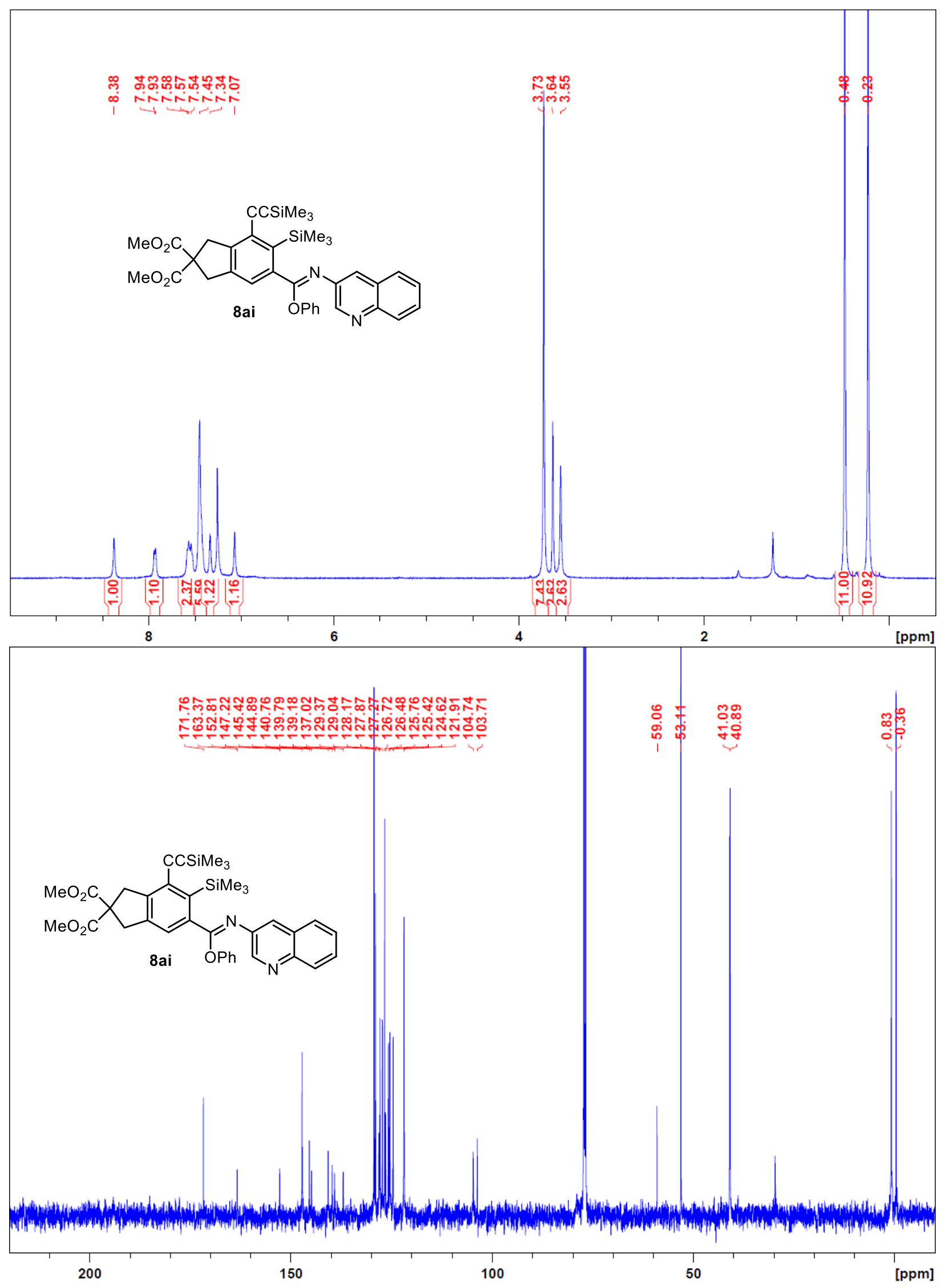




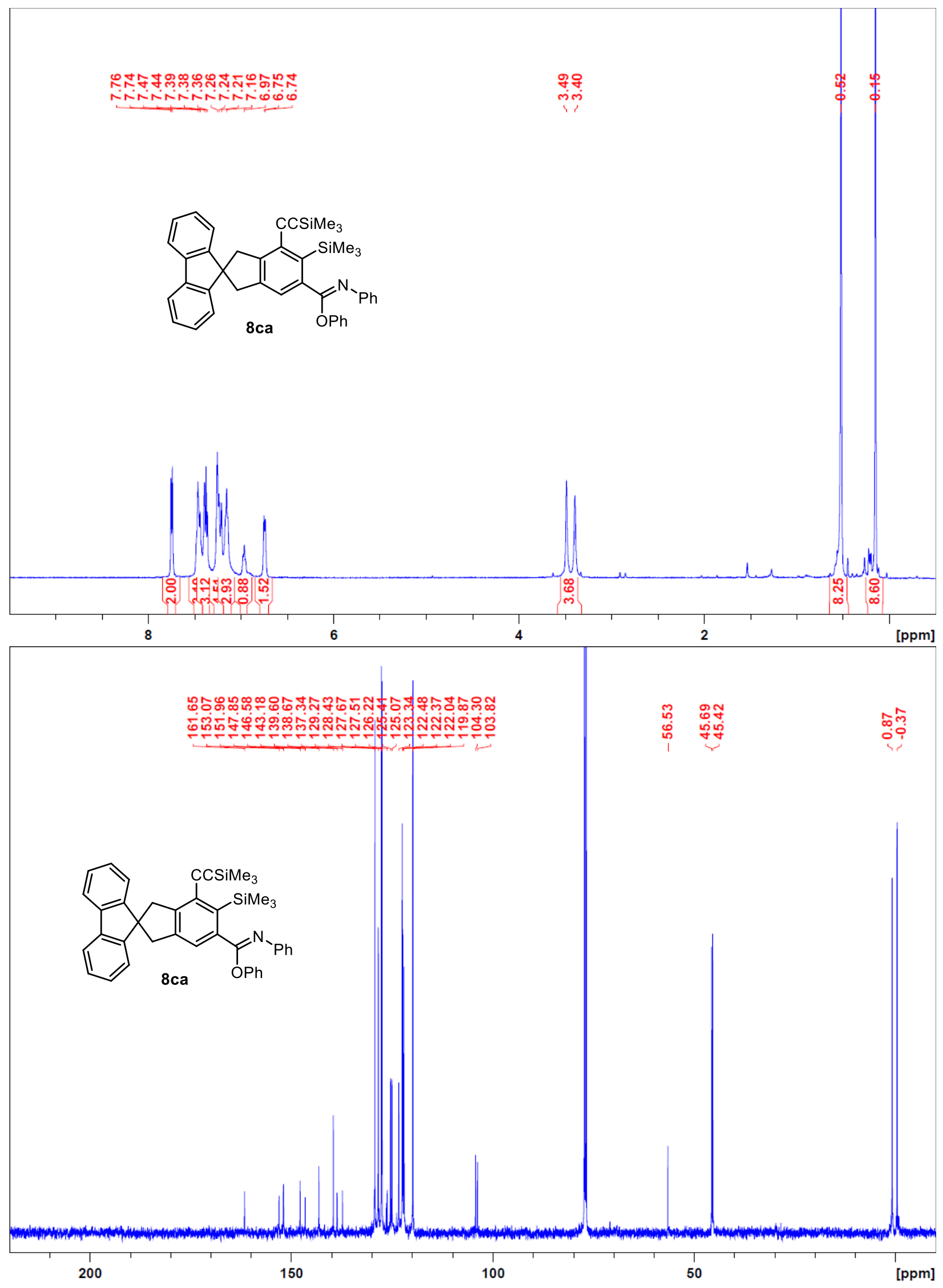

S52 


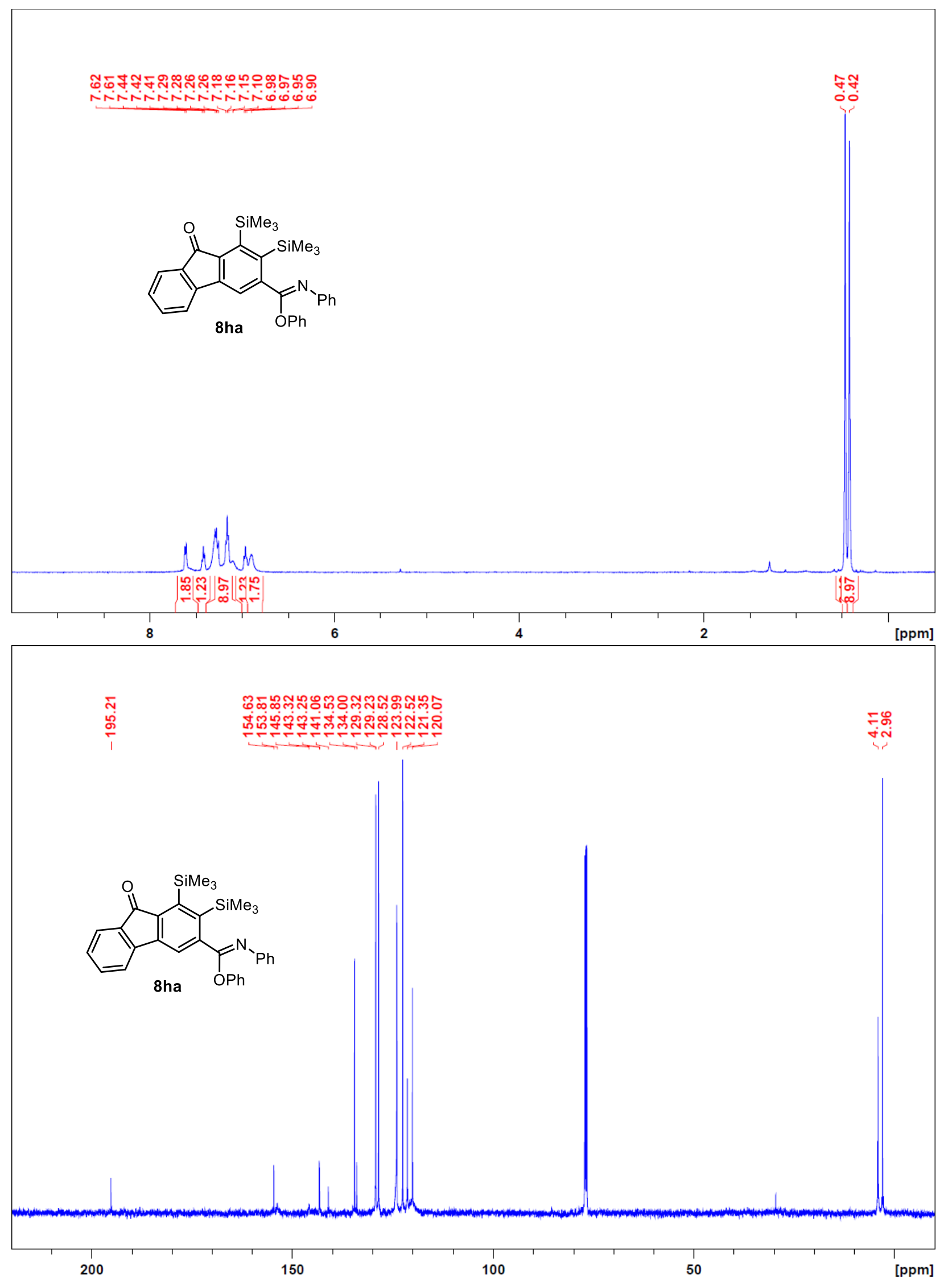



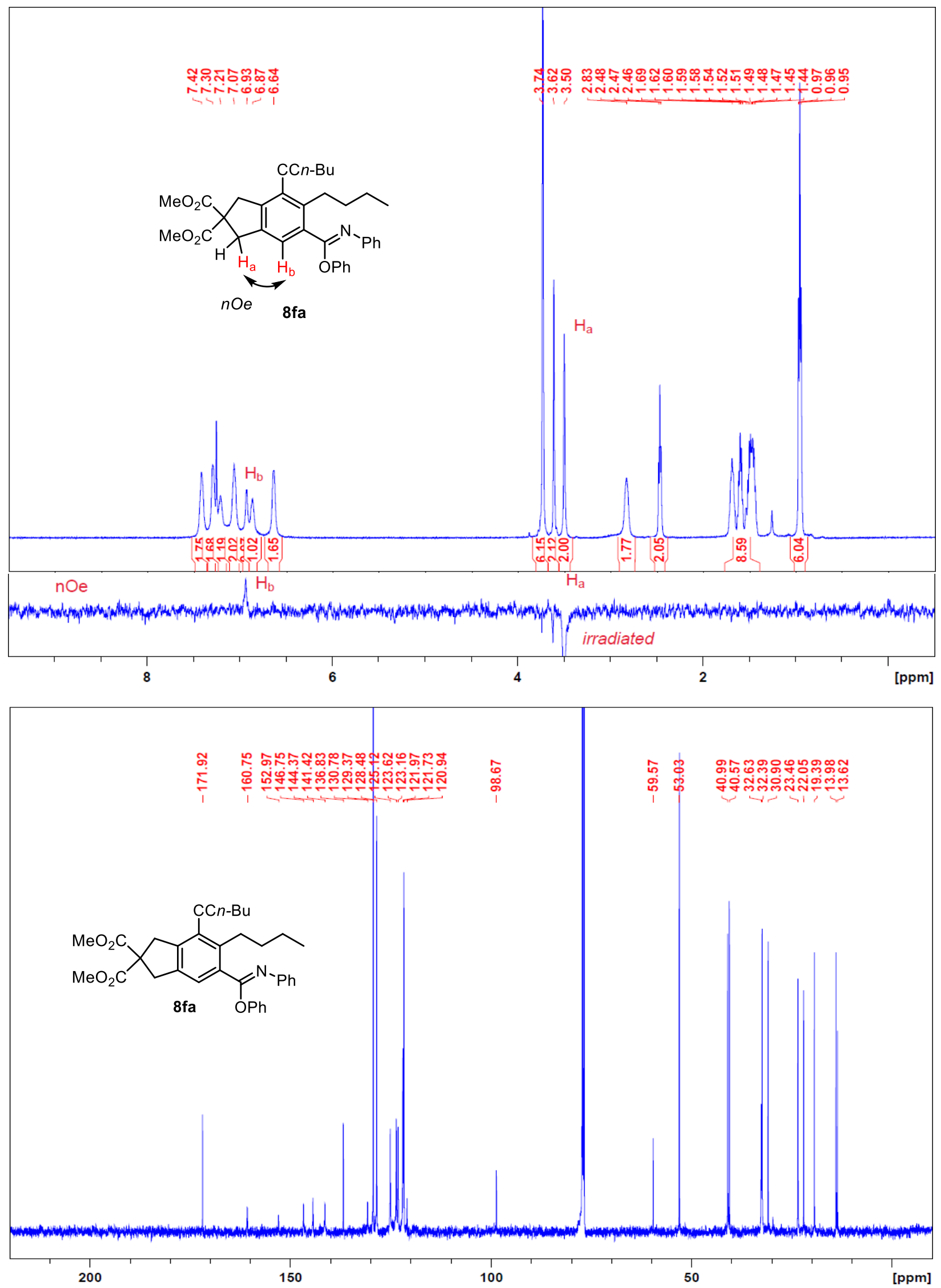


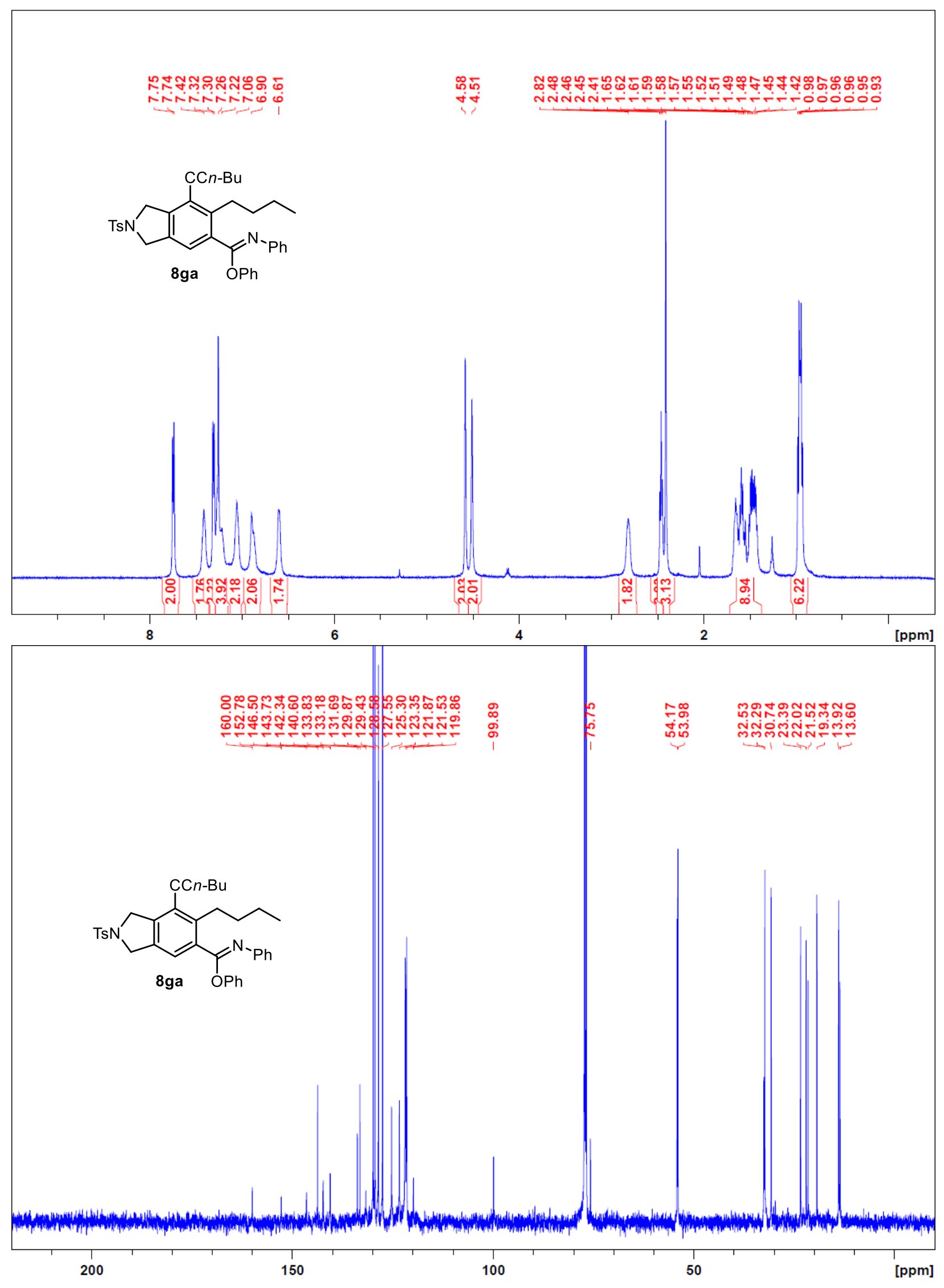




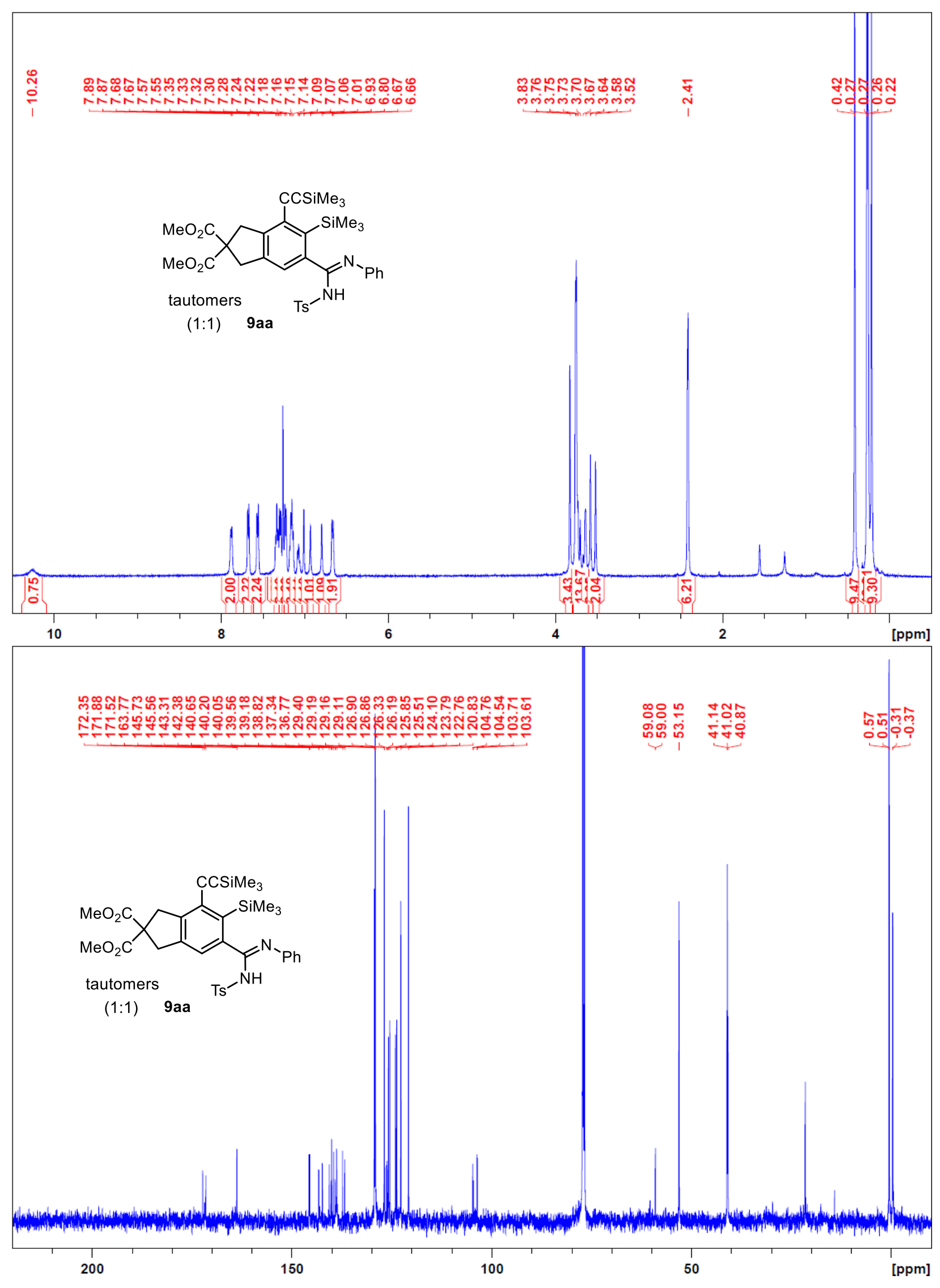



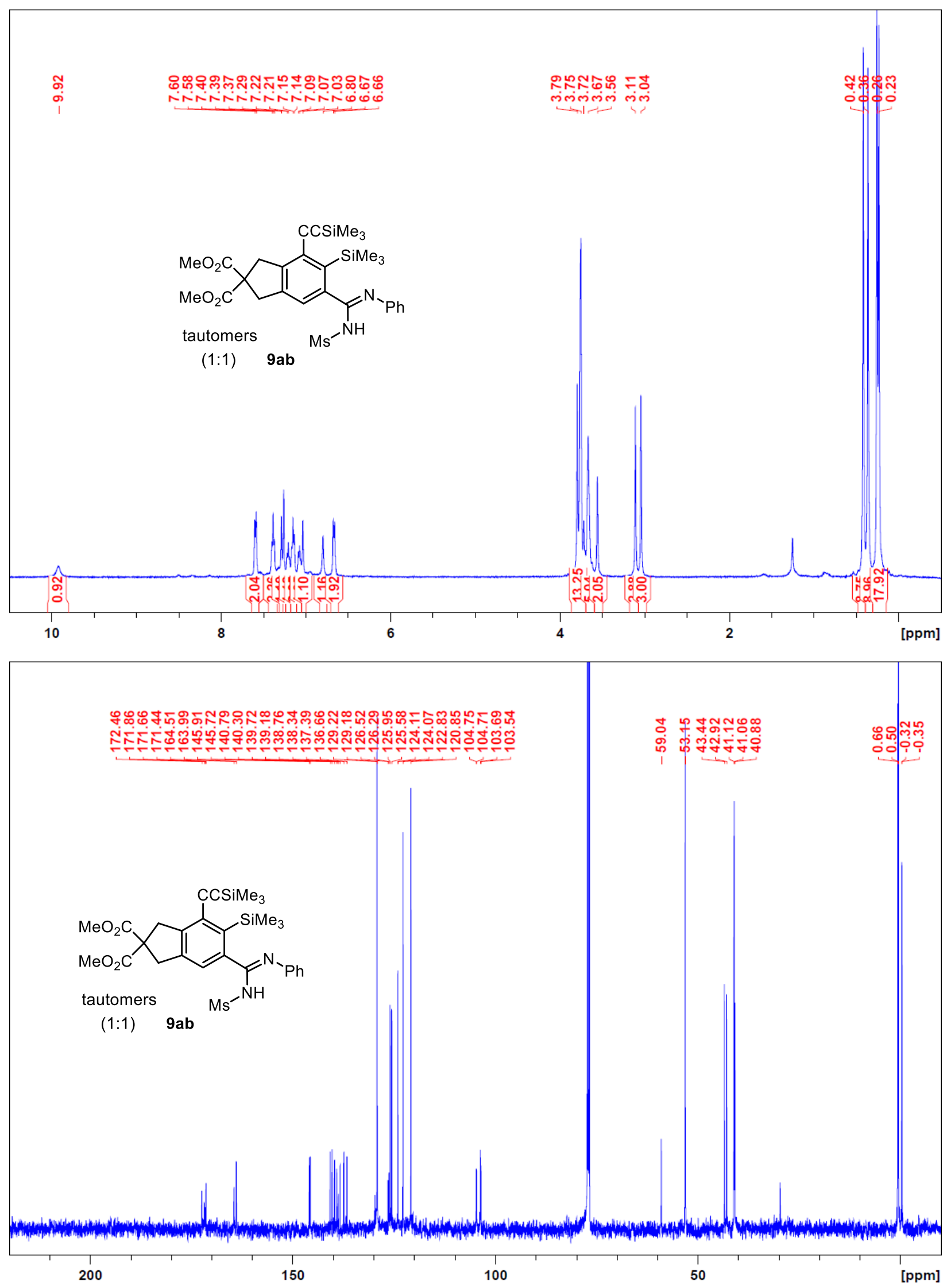

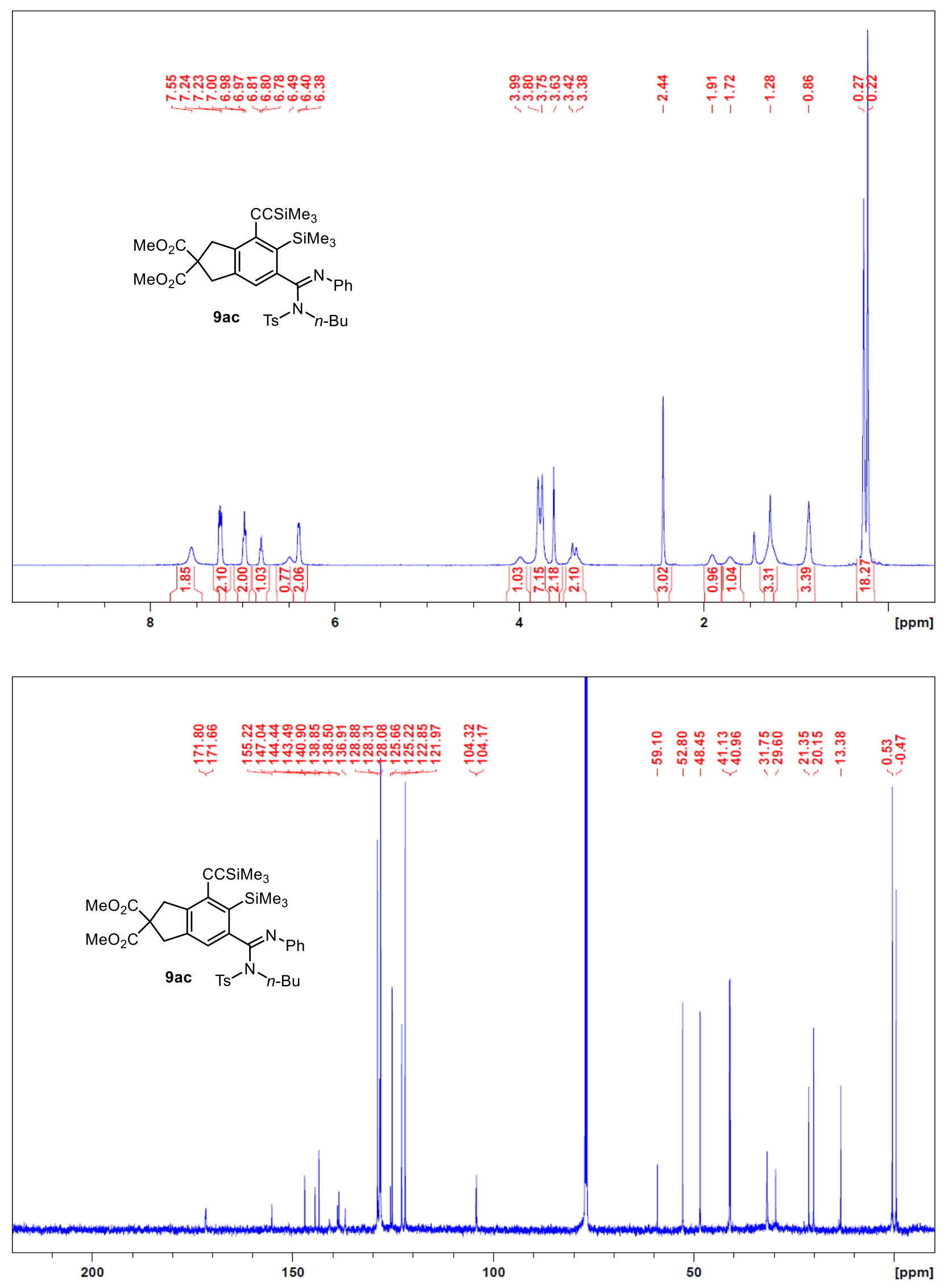


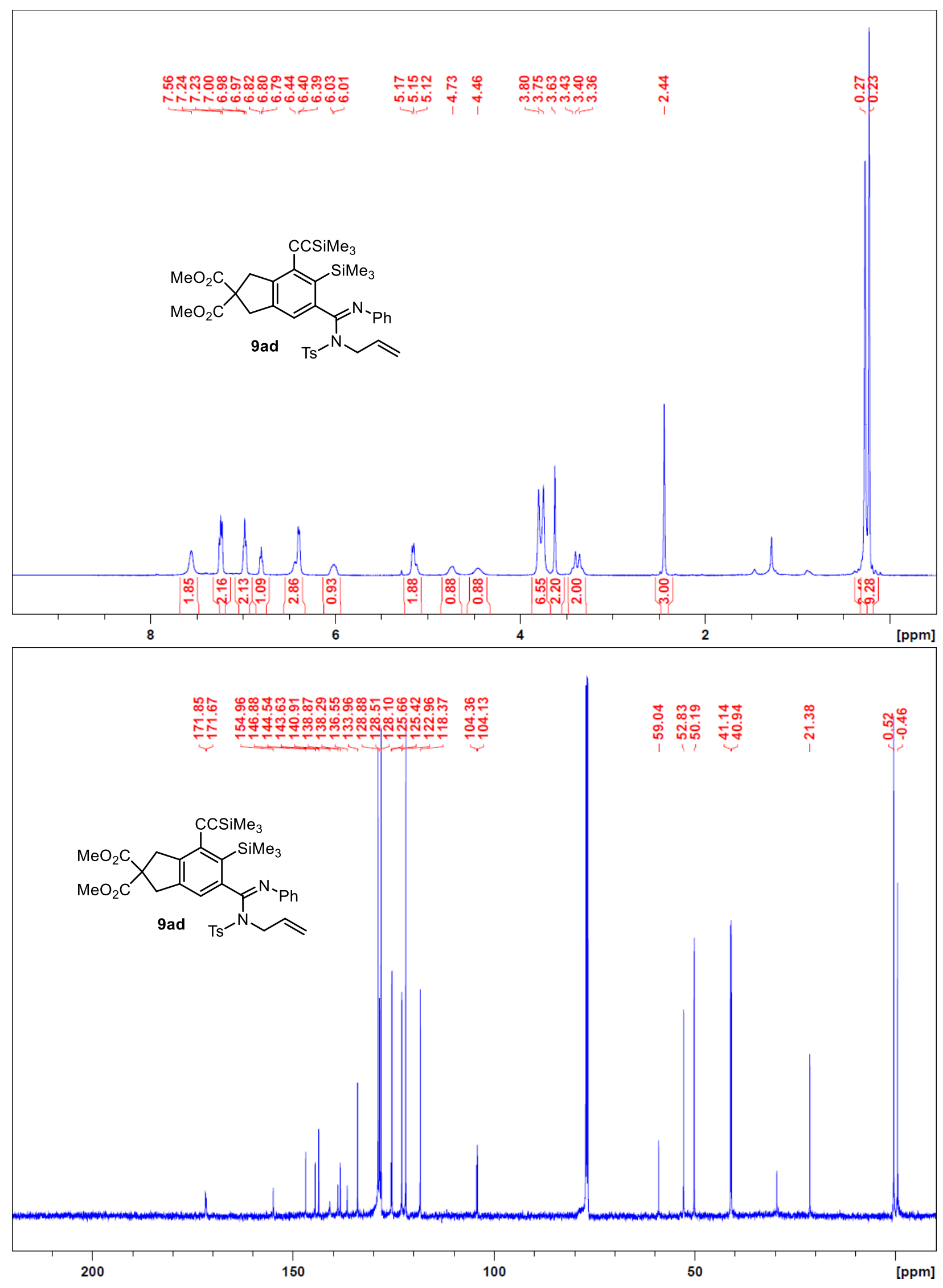



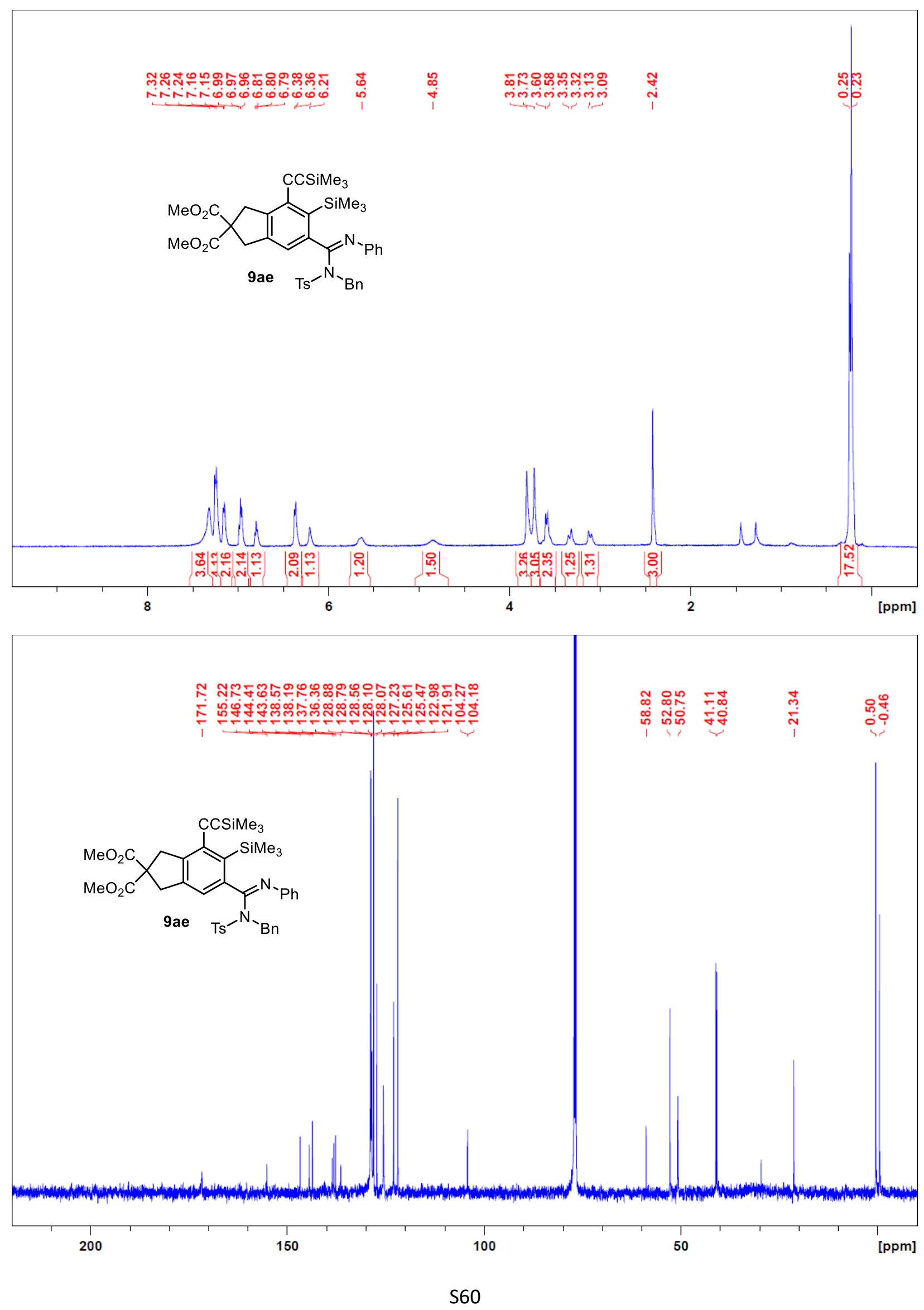

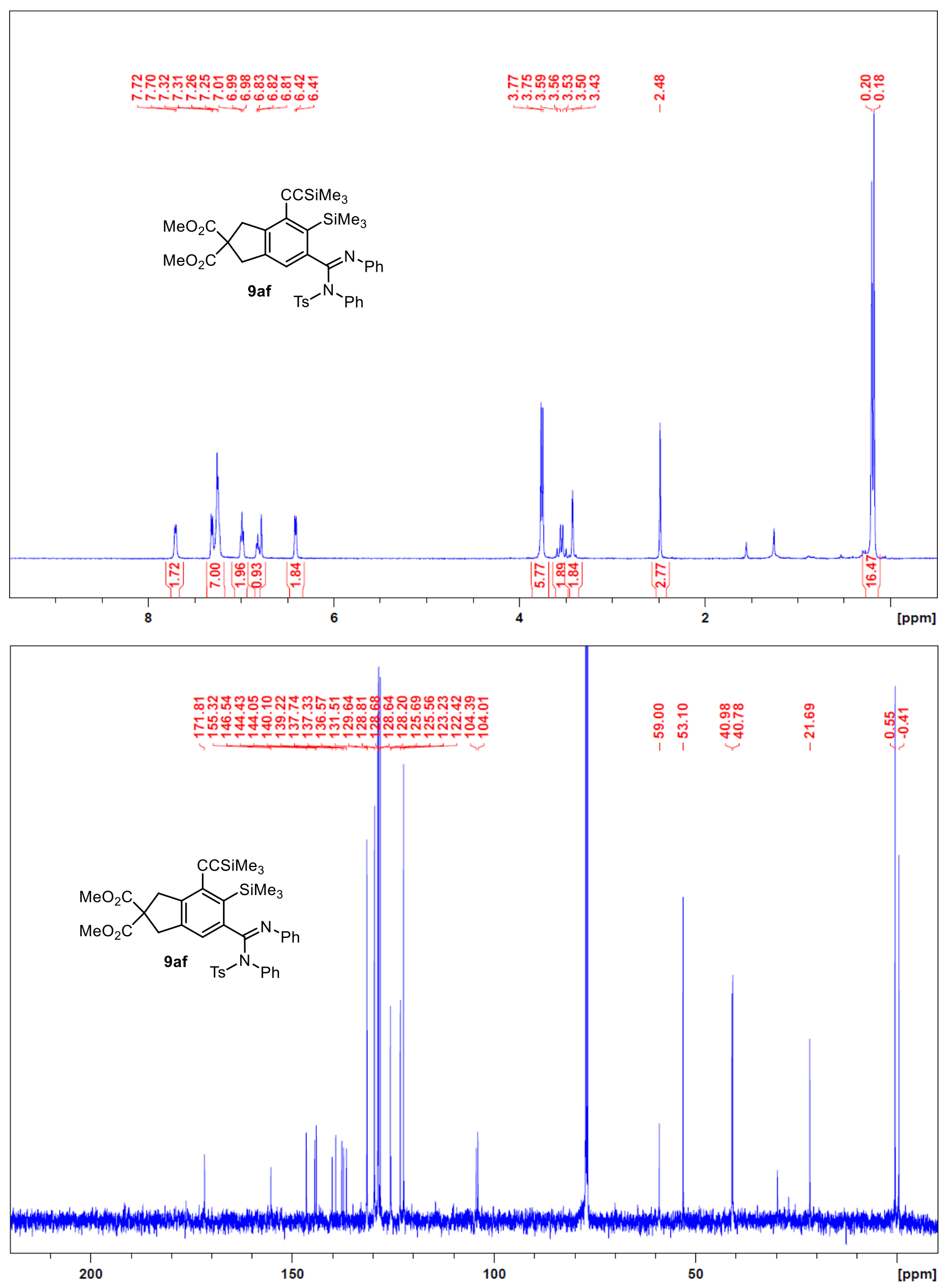


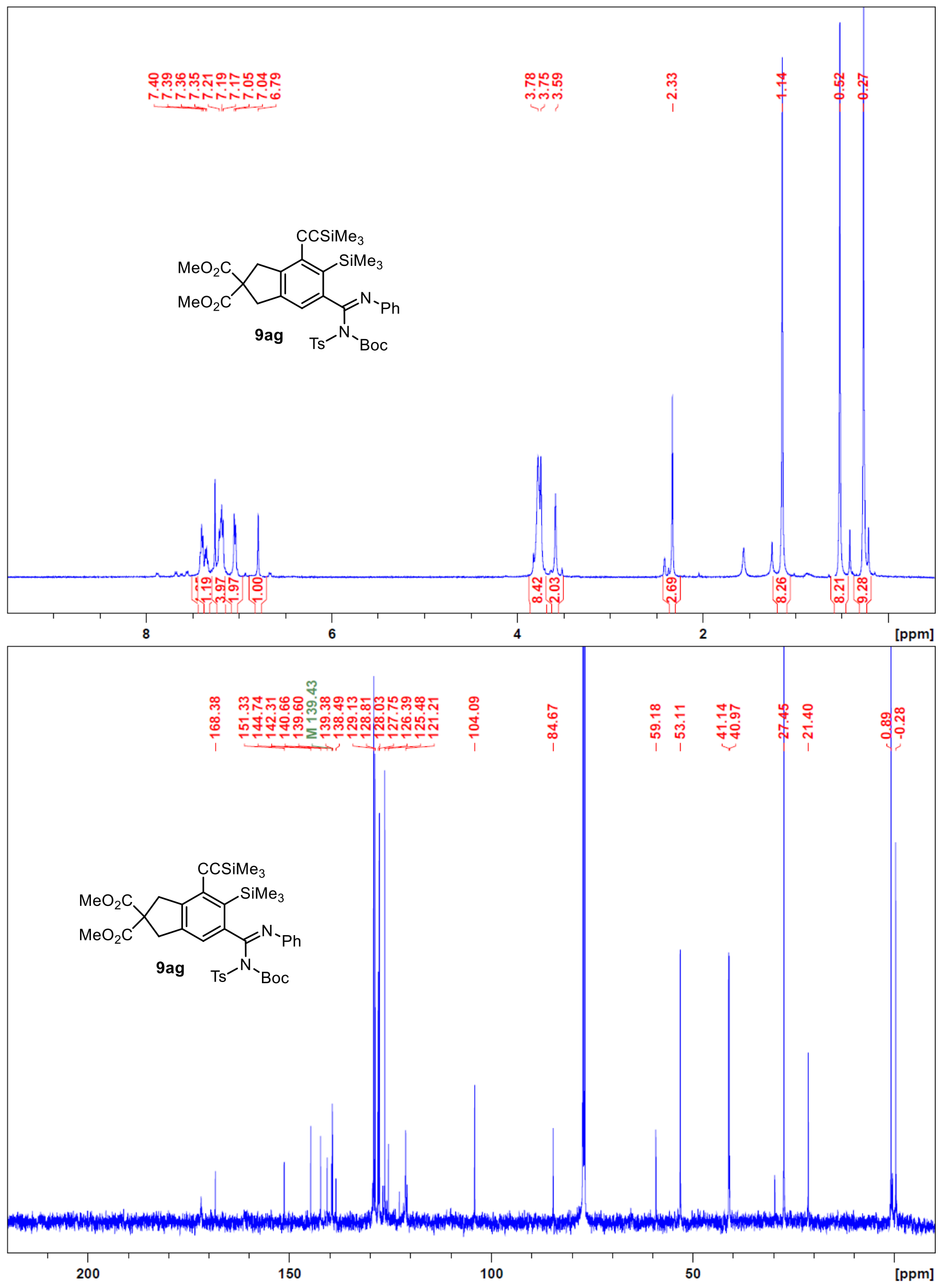




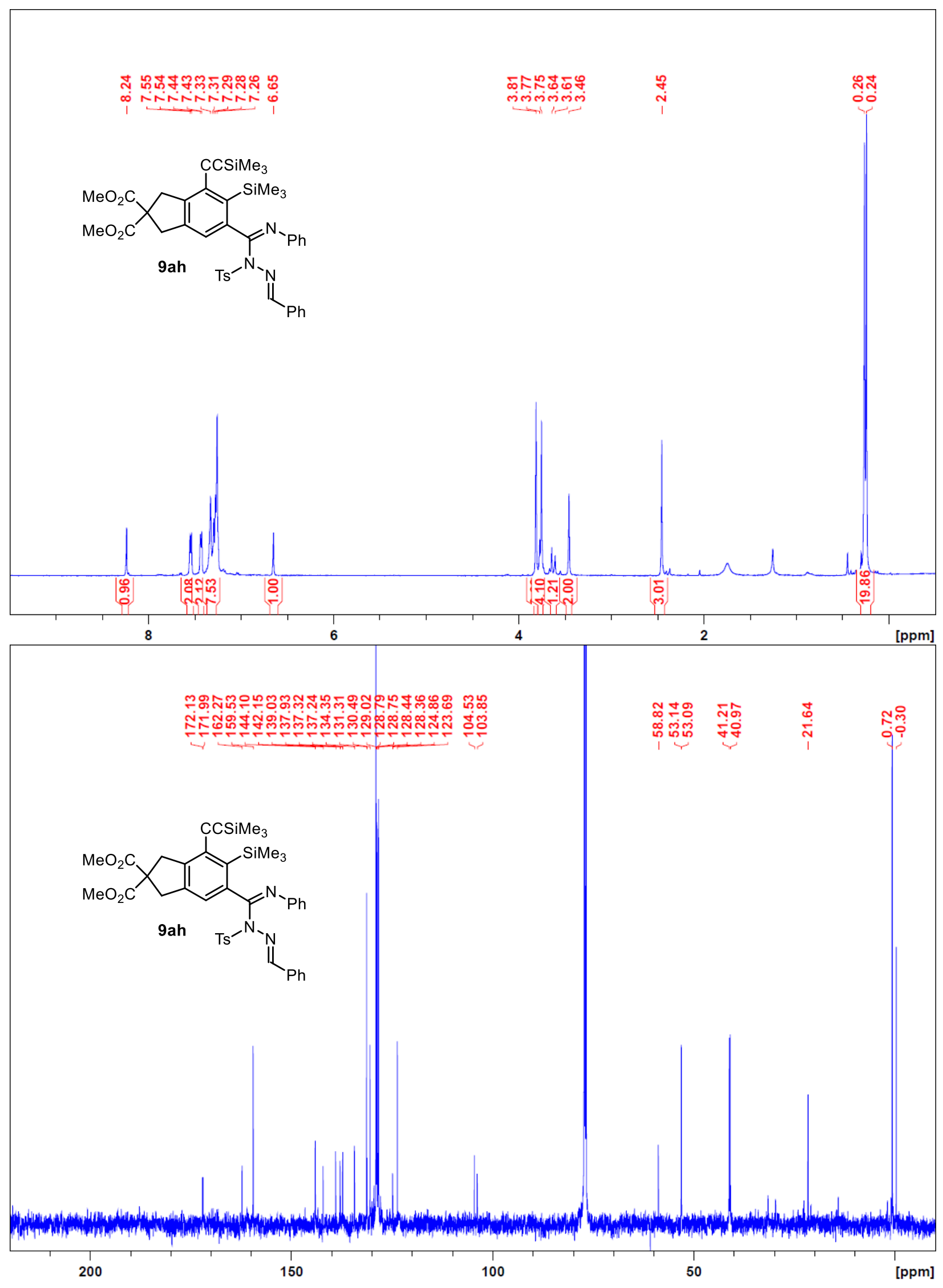



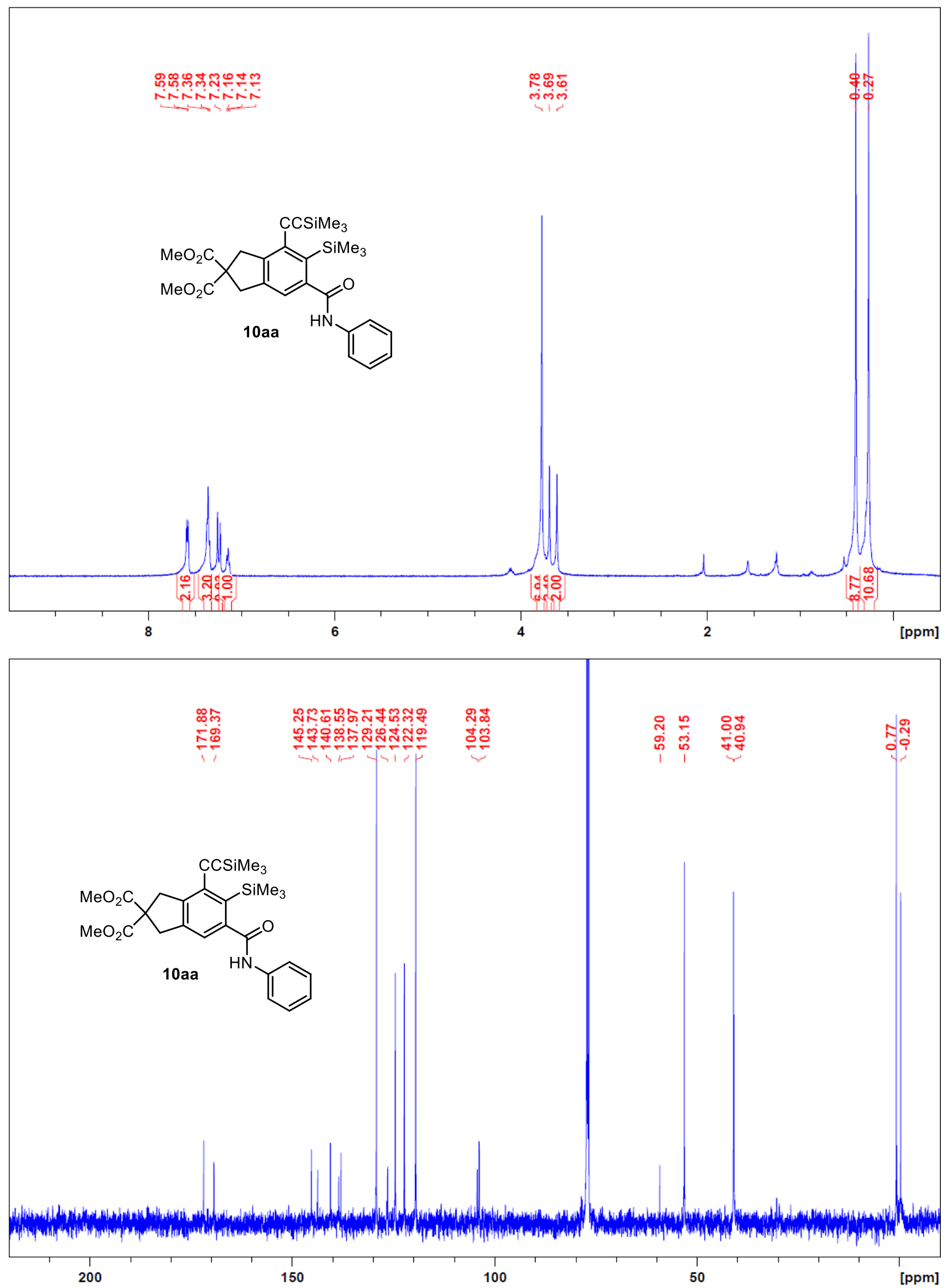

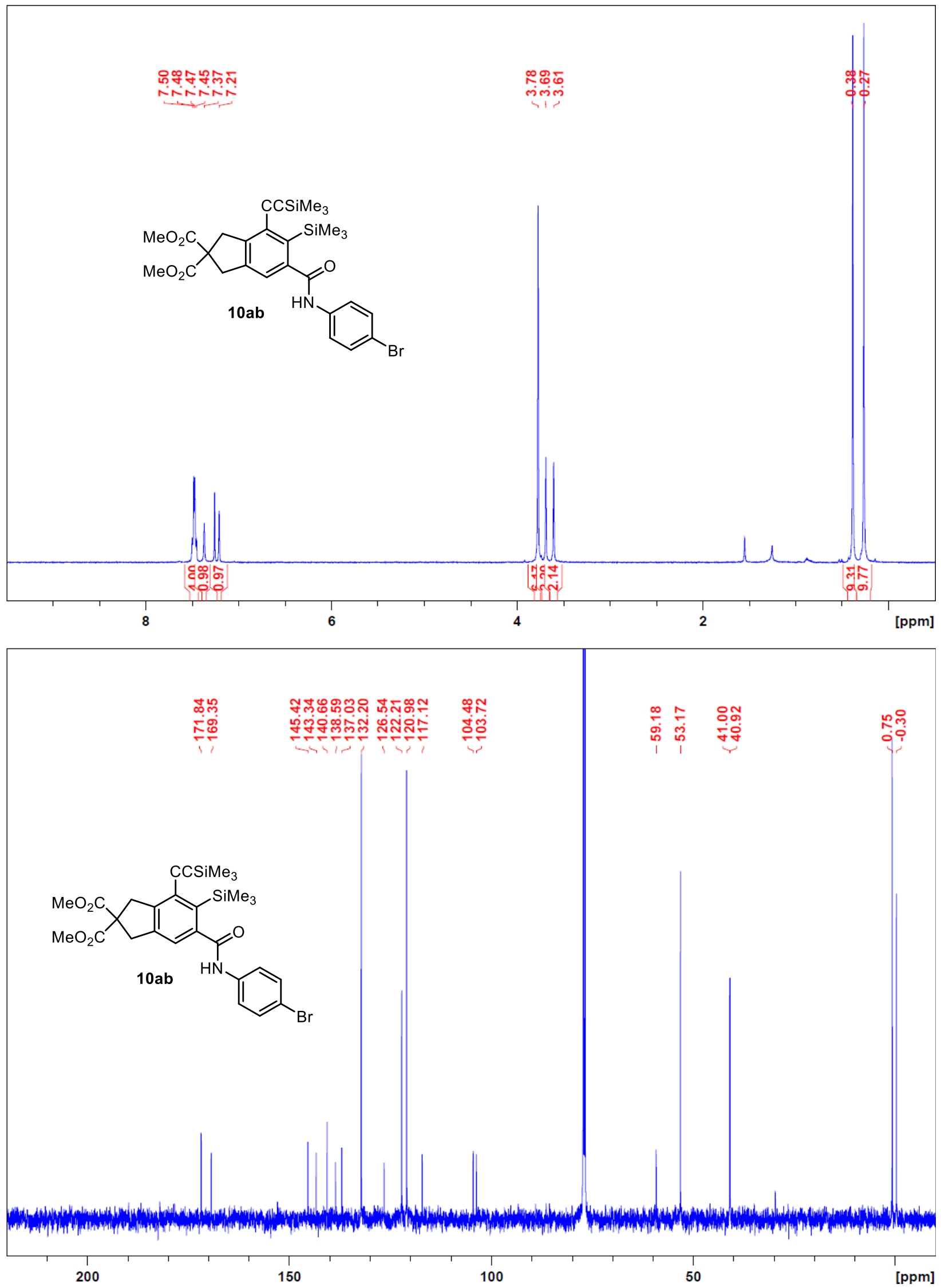

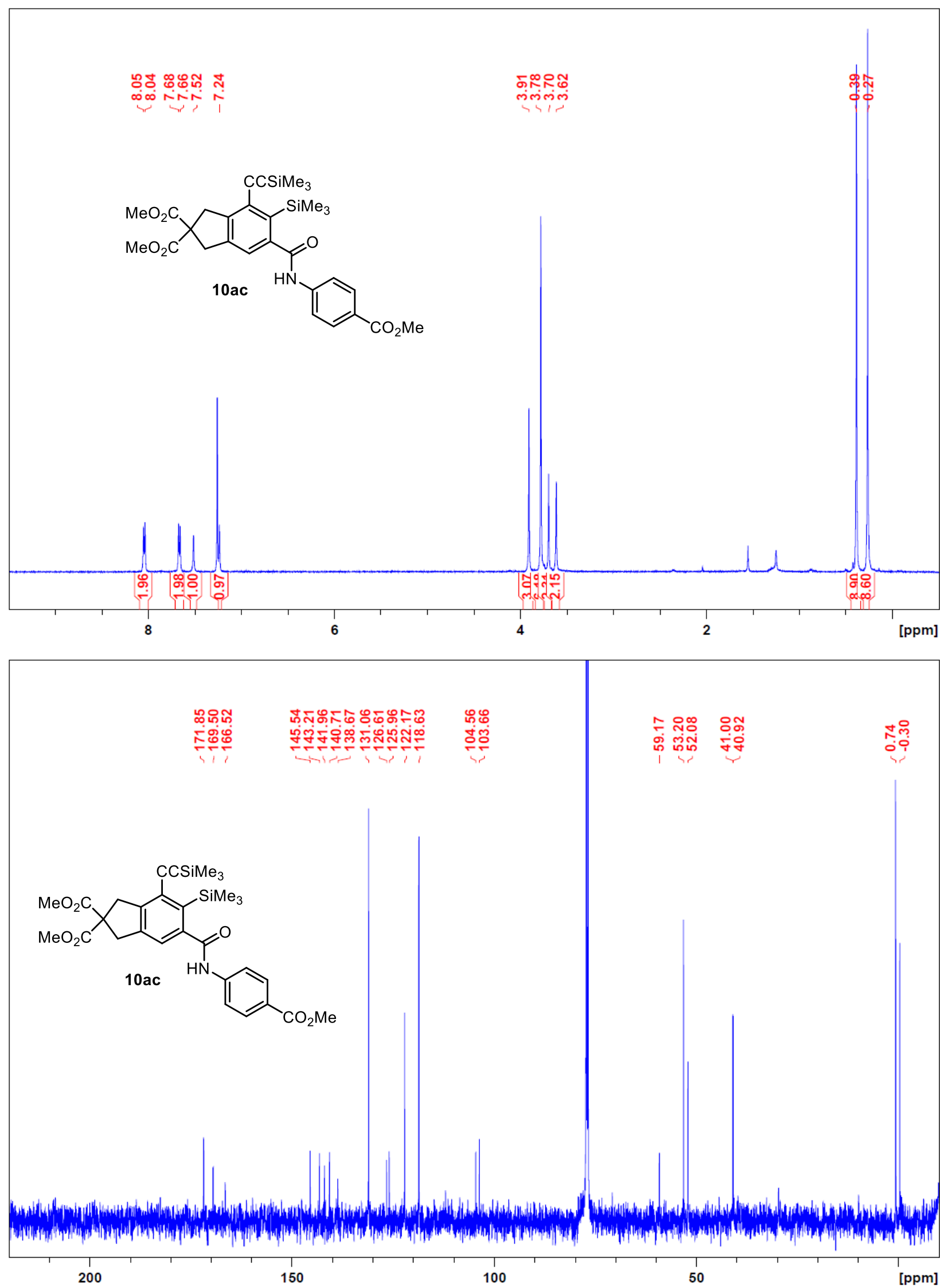

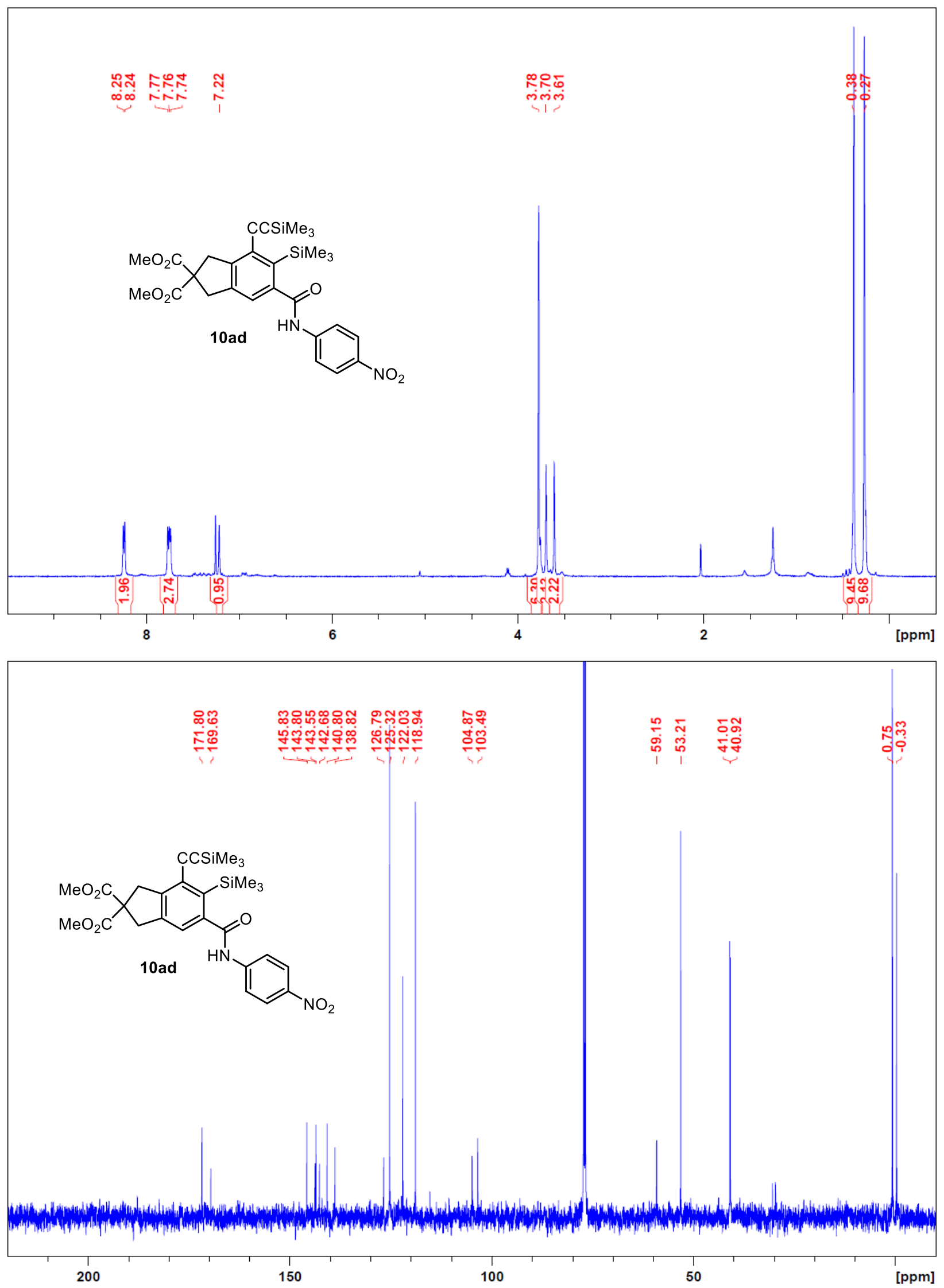

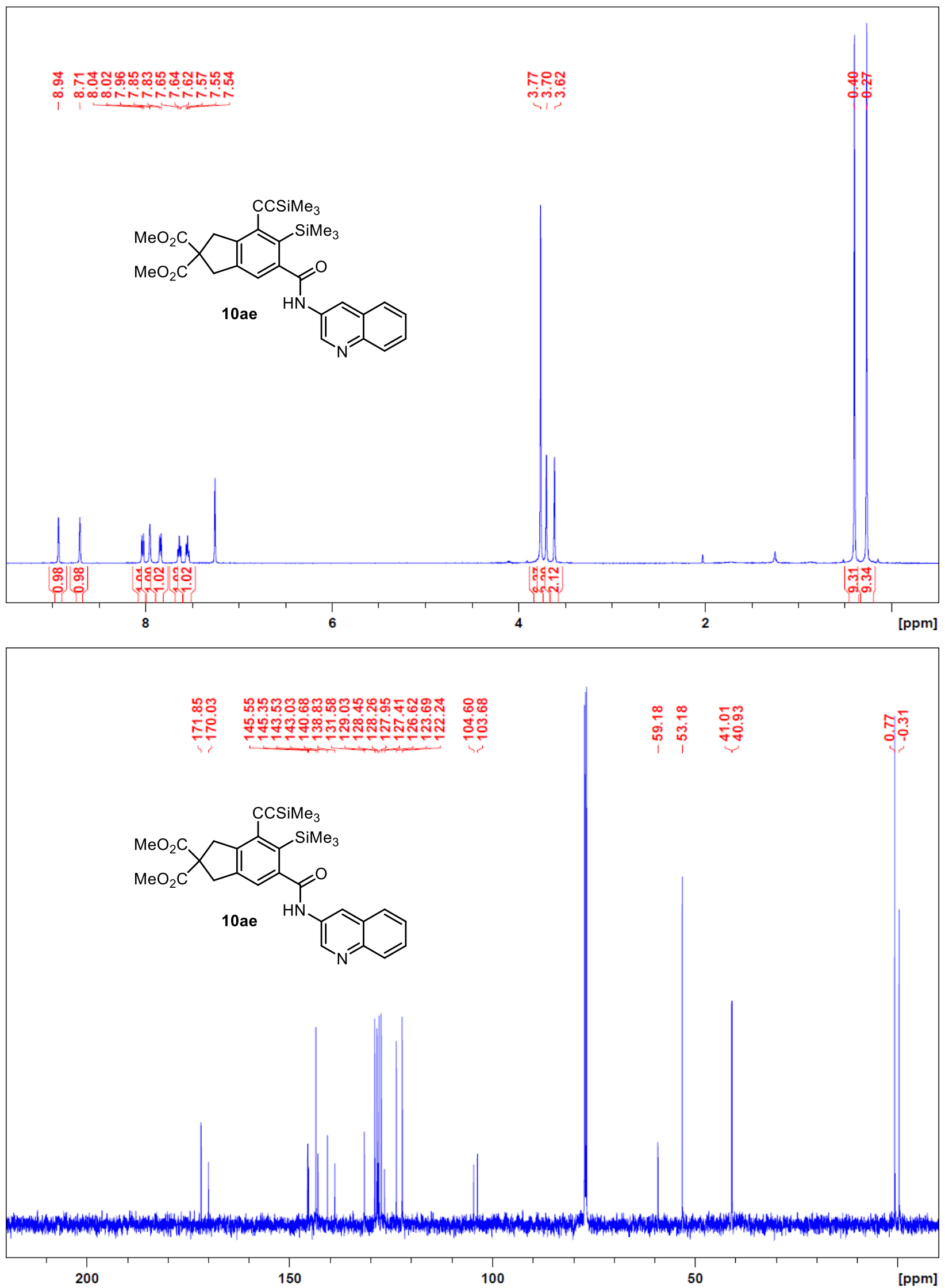


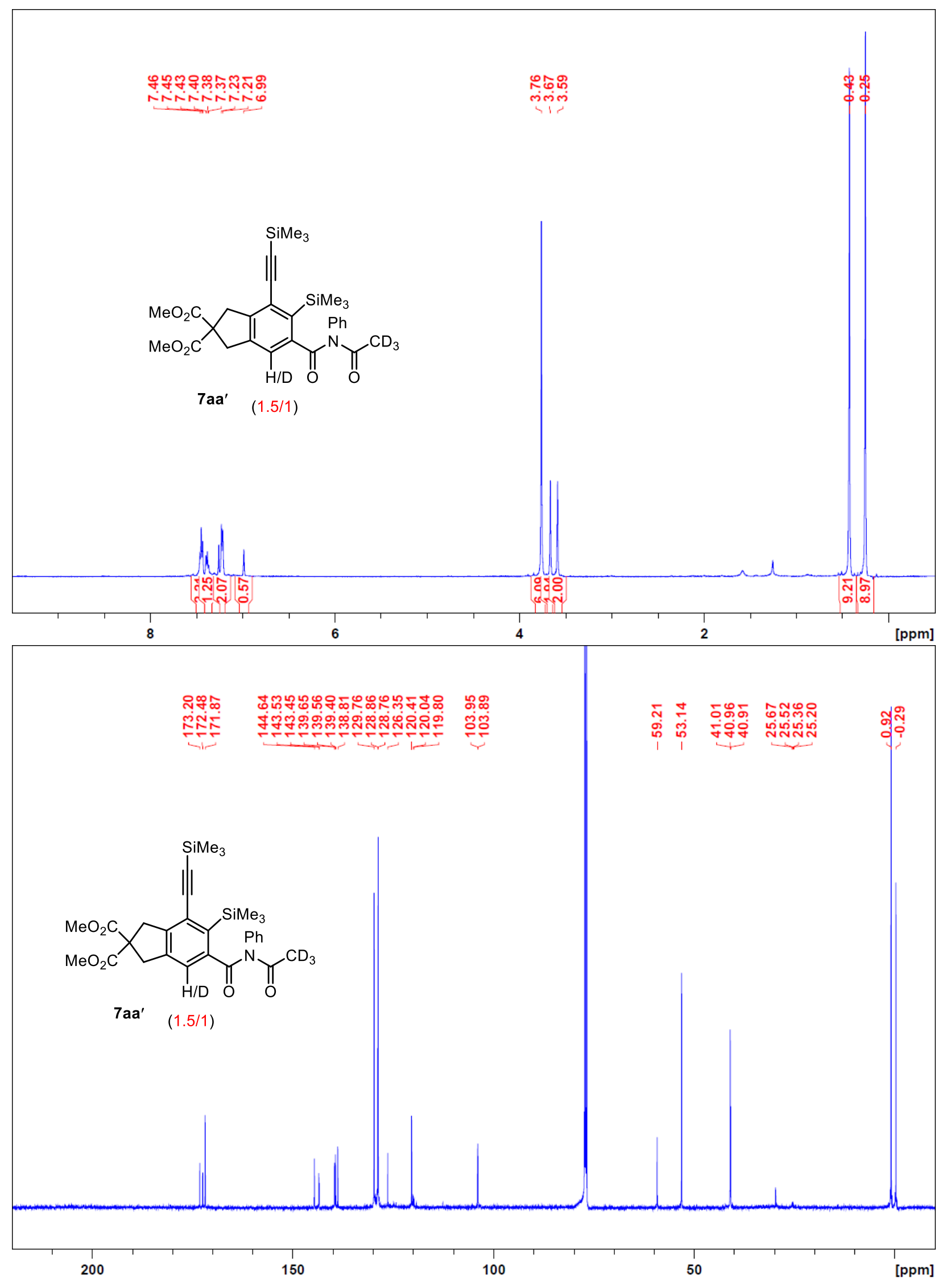

